\title{
Short-Latency Brain-Computer Interface Using Movement-Related Cortical Potentials
}

\section{Dissertation}

zur Erlangung des

mathematisch-naturwissenschaftlichen Doktorgrades

"Doctor rerum naturalium"

der Georg-August-Universität Göttingen

im Promotionsprogramm PCS

der Georg-August University School of Science

(GAUSS)

vorgelegt von

Ren Xu

aus Hunan, China

Göttingen 2016 
Betreuungsausschuss:

Prof. Dr. Dario Farina (Referent)

Prof. Dr. Xiaoming Fu (Koreferent)

Weitere Mitglieder der Prüfungskommission:

Prof. Dr. Carsten Damm

Prof. Dr. Jens Grabowski

Prof. Dr. Dieter Hogrefe

Prof. Dr. Hansjörg Scherberger

Tag der mündlichen Prüfung: June 24, 2016 


\section{Dedication}

The thesis is based on my work done at Department of Neurorehabilitation Engineering, University Medical Center Göttingen since October 2012. My work was financially supported by China Scholarship Council (Contract Number: 201204910155).

All the experiments conducted for this thesis were approved by the local ethical committees and the subjects signed an informed consent form before participation.

All chapters of this thesis were originally written by me. Part of the materials used in this thesis have also been published in journals or conferences, where I am the first author or corresponding author. The rights for reprint the published materials were obtained from the publisher.

Hereby I declare that I have written this thesis independently and with no other aids and sources than quoted.

Göttingen, May 28, 2016

Ren Xu 


\section{Abstract}

In recent years, brain-computer interface (BCI) has been developing as a communication and rehabilitation tool for patients with neurological diseases, such as stroke and spinal cord injury. This system decodes the user's intention from the brain signal, e.g. electroencephalography (EEG), and translates it into device commands. For either rehabilitation or communication system based on BCI, the latency of decoding algorithms is the fundamental factor which directly determines the system's performance.

In this thesis, movement-related cortical potentials (MRCP) were investigated as the signal modality for real-time detection of motor intention from EEG. Aiming at a short-latency brain switch based on MRCP, a manifold leaning based method was developed, yielding a true positive rate $>80 \%$ and a latency $<300$ ms, which significantly outperformed previous methods. Based on this brain switch, two closed-loop BCI systems were implemented for rehabilitation and communication purpose, respectively. In the rehabilitation system, the brain switch was used to trigger a motorized ankle-foot orthosis, mimicking real movement of dorsiflexion, for natural afferent feed- 
back. This closed-loop system was tested on healthy subjects, and neuroplasticity measured by transcranial magnetic stimulation was induced with only 15-min intervention, indicating an efficient neuromodulation tool for rehabilitation. Further, the brain switch was extended to a multi-class BCI for communication purpose, by combining an electrotactile menu. The online testing on healthy subjects demonstrated its desirable properties of gazeindependence, high information transfer rate, and vast applicability. Finally a pre-clinical measurement was performed on spinal cord injured patients for investigation of the MRCP features, as a first step of potential application of the proposed BCI systems on this group of patients.

In conclusion, the main out-come of this thesis is a short-latency brain switch (Chapter 3) based on the investigation of MRCPs (Chapter 2), which was implemented in closed-loop BCI systems for rehabilitation and communication (Chapter 4), with potential application on patients with stroke and spinal cord injury (Chapter 5). 


\section{Acknowledgments}

First of all, I would like to express my greatest thanks to my supervisor Prof. Dr. Dario Farina. His forward guidance was the most invaluable input for my thesis. I would also like to than Prof. Dr. Xiaoming Fu, whose support made it possible to start my $\mathrm{PhD}$ study at Göttingen.

Further, I thank Dr. Ning Jiang, who headed the BCI lab and directly tutored me for the past three years. He provided more assistance than I could ever request, and those fruitful discussion with him always led my work to a brand-new stage.

Moreover, I thank all my colleagues and collaborators, without whom I could not finish all these studies in the thesis so smoothly. Particularly, I thank Dr. Chuang Lin, who opened the door of machine learning for my BCI studies. And I thank Prof. Dr. Natalie Mrachacz-Kersting, whose exceptional expertise in neurophysiology was an indispensable help for my work.

Last but not least, I thank my whole family for their constant support. Special thanks go to my wife, for her accompanying and understanding which inspired me to get so far. 


\section{Table of Contents}

Dedication $\quad$ ii

Abstract iii

Acknowledgments $\quad$ v

Table of Contents ix

List of Tables $\quad$ x

List of Figures $\quad$ xii

List of Abbreviations xiii

1 Introduction 1

1.1 Background ...................... 1

1.1.1 Brain-computer interface and its applications . . . . . 2

1.1.2 Current sate of the art . . . . . . . . . . . 2

1.2 Real-time brain-computer interface platform . . . . . . . 5 
1.2 .1 Hardware ................. . . 5

1.2 .2 Software .................. . . 7

1.3 Thesis structure . . . . . . . . . . . . . . . 8

$2 \quad$ MRCP morphology 11

2.1 The influence of the cue . . . . . . . . . . . . 12

2.1.1 Subjects and experiment ............. 12

2.1.2 Signal analysis . . . . . . . . . . . . . 12

2.1.3 Results................... 13

2.2 The influence of the motor task . . . . . . . . . 16

2.2.1 Experiment .................. 17

2.2.2 Comparison of MRCPs . . . . . . . . . . . 18

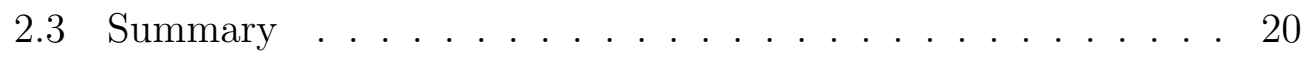

3 Short-latency brain switch $\quad 21$

3.1 Real-time detection of MRCP . . . . . . . . . . . 22

3.1.1 Locality preserving projection ........... 23

3.1.2 Offline parameter tuning . . . . . . . . . . 26

3.1.3 Online experiment ................. 31

3.1.4 Online performance . . . . . . . . . . . 39

3.1.5 Discussion . . . . . . . . . . . . . . . 40

3.2 Factors that influence the performance of the short-latency brain switch ................... . . 43

3.2.1 Motivation ................. 43 
3.2 .2 Methods . . . . . . . . . . . . . . . . . . 44

3.2.2.1 EEG processing algorithm . . . . . . . . . 45

3.2.2.2 Statistical methods . . . . . . . . . . . . . 48

3.2 .3 Results. . . . . . . . . . . . . . . . . . 48

3.2.3.1 Signal Morphology . . . . . . . . . . . . . . . 48

3.2.3.2 BCI performance . . . . . . . . . . . . 50

3.2.4 Discussion . . . . . . . . . . . . . . . . . . . 54

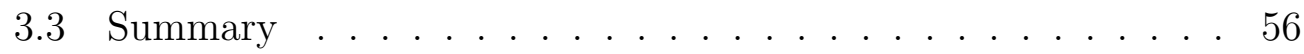

4 Closed-loop BCI applications $\quad 57$

4.1 Brain switch driven mechanical stimulation for rehabilitation . 58

4.1 .1 Motivation . . . . . . . . . . . . . 58

4.1 .2 System development . . . . . . . . . . . . . . 60

4.1 .3 BCI-MAFO intervention . . . . . . . . . . . . 61

4.1.4 The effect on MRCP morphlogy . . . . . . . . . . . . 63

4.1.5 The effect on neural plasticity . . . . . . . . . . 66

4.1.6 Comparison with BCI-ES . . . . . . . . . . . 75

4.1 .7 Discussion . . . . . . . . . . . . . . . . 76

4.2 Brain switch triggered selective electro-tactile menu for communication . . . . . . . . . . . . . . . 81

4.2 .1 Motivation . . . . . . . . . . . . . . . 81

$4.2 .2 \quad$ BCI menu setup . . . . . . . . . . . . . . . . 83

$4.2 .3 \quad$ Experimental Procedure . . . . . . . . . . . . . 87 
4.2.4 Evaluation criteria .............. . 90

4.2 .5 Results . . . . . . . . . . . . . . . . 92

4.2.6 Discussion .................. . . 96

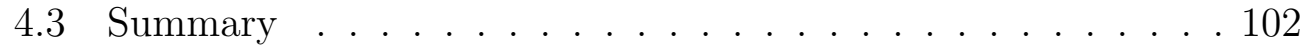

5 Clinical measurement $\quad 104$

5.1 MRCP in paraplegic patients . . . . . . . . . . 104

5.1.1 Motivation ................... 104

5.1.2 Subjects and experiment . . . . . . . . . . 107

5.1.3 MRCP morphology in paraplegic patients . . . . . . 111

5.1.4 Classification of MRCP . . . . . . . . . . . 117

5.1 .5 Discussion ................... 123

6 Summary and future work 131

6.1 Further improvement of the brain switch . . . . . . . . . 132

6.2 Clinical applications . . . . . . . . . . . . . . . . . 134

$\begin{array}{ll}\text { Bibliography } & 135\end{array}$

$\begin{array}{ll}\text { Vita } & 147\end{array}$ 


\section{List of Tables}

3.1 Offline performance ..................... 28

3.2 Brain switch performance with different factors . . . . . . . 52

4.1 BCI performance . . . . . . . . . . . . 72

4.2 Detection performance . . . . . . . . . . . . . . . . 94

5.1 Patient information . . . . . . . . . . . . 108 


\section{List of Figures}

1.1 EEG channel locations . . . . . . . . . . . . . 6

1.2 Experimenter View . . . . . . . . . . . . . . 7

2.1 Visual and auditory cue . . . . . . . . . . . . . . . 13

2.2 MRCPs with visual and auditory cue . . . . . . . . . . 14

2.3 Magnitude of negative peak . . . . . . . . . . . . 15

2.4 Timing of negative peak in MRCP . . . . . . . . . . 16

2.5 Experiment procedure with different motor tasks . . . . . . 17

2.6 MRCPs from ballistic and repetitive tasks . . . . . . . . 19

3.1 Three steps for LPP . . . . . . . . . . . . . . 25

3.2 Single trial MRCP of self-paced dorsiflexion . . . . . . . . 26

3.3 Offline performance with change of reduced dimension . . . . . 29

3.4 Representative detection output . . . . . . . . . . . . . 31

3.5 Online experiment flow . . . . . . . . . . . . . 32

3.6 LPP-LDA classifier . . . . . . . . . . . . . . 34

3.7 Online performance . . . . . . . . . . . . . 40 
3.8 SMR mapping . . . . . . . . . . . . . . . . . . . . . . . . . 49

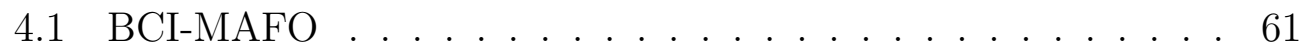

4.2 MRCP change after intervention . . . . . . . . . . 65

4.3 BCI-MAFO experiment protocol . . . . . . . . . . . 67

4.4 MEP change agter intervention: single subject . . . . . . . 71

4.5 Comparison with controls . . . . . . . . . . . . . 73

4.6 Stretch reflex and active MEP . . . . . . . . . . . . . . 74

4.7 Comparison with BCI-ES $\ldots \ldots \ldots \ldots 77$

4.8 ES locations . . . . . . . . . . . . . . . . . . . . . 85

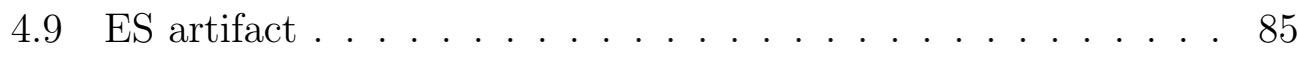

4.10 BCI menu setup . . . . . . . . . . . . . . . . 87

4.11 BCI menu experiment . . . . . . . . . . . . . . . . 90

4.12 MRCP morphology with/without ES . . . . . . . . . . 93

4.13 Performance of 4-class BCI menu . . . . . . . . . . . . 95

4.14 Performance of 8-class BCI menu $\ldots \ldots \ldots$. . . . . . 96

5.1 Clinical experiment protocol . . . . . . . . . . . . . . 109

5.2 MRCP morphology and statistical comparison for motor im-

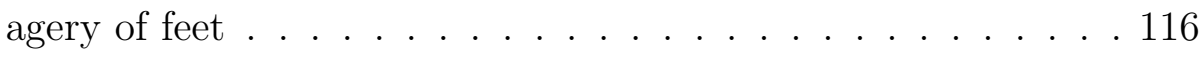

5.3 MRCP morphology and statistical comparison for motor imagery of right hand . . . . . . . . . . . . . . 117

5.4 Classification accuracy for feet . . . . . . . . . . 120

5.5 Classification accuracy for right hand . . . . . . . . . . 121 


\title{
List of Abbreviations
}

\author{
ANOVA Analysis of variance \\ ASIA American spinal injury association \\ aPF Active false positive \\ BCI Brain-computer interface \\ CNV contigent negative variation \\ CNP Central neuropathic pain \\ CNS Central neural injury \\ CP Patient with complete injury \\ DL Detection latency \\ EEG Electroencephalography \\ EMG Electromyography \\ EOG Electrooculography \\ ERD Event-related desynchronization \\ ERS Event-related synchronization \\ ES Electrical stimulation \\ FES Functional electrical stimulation
}




\begin{tabular}{|c|c|}
\hline FP & False positive \\
\hline GUI & Graphic user interface \\
\hline $\mathrm{HV}$ & Healthy volunteer \\
\hline IP & Patient with incomplete injury \\
\hline ITR & Information transfer rate \\
\hline $\mathrm{kNN}$ & k-nearest-neighborhood \\
\hline LDA & Linear discriminant analysis \\
\hline LH & Left hand \\
\hline LPP & Locality preserving projection \\
\hline LTP & Long term potential \\
\hline $\mathrm{MAFO}$ & Motorized ankle-foot orthosis \\
\hline $\mathrm{ME}$ & Movement execution \\
\hline MEP & Motor evoked potential \\
\hline $\mathrm{MF}$ & Matched filter \\
\hline MI & Motor imagery \\
\hline $\mathrm{MRCP}$ & Movement-related cortical potential \\
\hline MVC & Maximum voluntary contraction \\
\hline NI & National Instrument \\
\hline PCA & Principal component analysis \\
\hline $\mathrm{pFP}$ & Passive false positive \\
\hline $\mathrm{PNP}$ & Patient with no pain \\
\hline PSD & Power spectral density \\
\hline PWP & Patient with pain \\
\hline
\end{tabular}




$\begin{array}{ll}\text { RH } & \text { Right hand } \\ \text { RMS } & \text { Root mean square } \\ \text { ROC } & \text { Receiver operating characteristic } \\ \text { RTh } & \text { Resting threshold } \\ \text { SCI } & \text { Spinal cord injured } \\ \text { SLR } & \text { Short latency response } \\ \text { SMR } & \text { Sensory motor rhythm } \\ \text { SSEP } & \text { Steady-state evoked potential } \\ \text { TA } & \text { Tibialis Anterior } \\ \text { TMS } & \text { Transcranial magnetic stimulation } \\ \text { TPR } & \text { True positive rate } \\ \text { VNS } & \text { Visual numerical scale } \\ \text { WN } & \text { Window number }\end{array}$




\section{Chapter 1}

\section{Introduction}

\section{$1.1 \quad$ Background}

According to the figure from the World Health Organization, more than one billion people worldwide suffer from central neural system diseases, such as stroke and spinal cord injury [90]. Even though current surgical or pharmaceutical treatments can help the patients to survive, their quality of life seriously decreases due to after-effects which cannot be completely treated. For example, approximately $65 \%$ of stroke survivors have severely physical disabilities, even after medical treatment and following rehabilitation therapy [81].

Apart from the traditional physical therapy, in the past few decades, new methods have been emerged, including functional electrical stimulation(FES) [64], virtual reality [44] and robotic therapy [42]. Following this line, brain- 
computer interface (BCI) has recently attracted widespread attentions [18], with the promise to assist or rehabilitate the lost sensory or motor functions.

\subsubsection{Brain-computer interface and its applications}

A BCI builds a pathway between the brain and external devices, bypassing the peripheral pathway, for assisting or restoring impaired sensory or motor functions [18]. In the past few decades, BCI researchers mainly focused on its application for communication [89], such as a BCI speller [28]. More recently, the BCI based applications in rehabilitation have attracted great attention, and several clinical studies have been performed [4][73][54].

Due to its non-invasive property and relatively low cost, electroencephalography (EEG) is the majority brain signal for BCI studies. Several signal modalities in EEG have been previously investigated, such as visual evoked potentials, sensory-motor rhythms, and slow cortical potentials [18].

\subsubsection{Current sate of the art}

Among EEG modalities for BCI, P300 is the most successful one and it has numerous applications [23]. It has been well documented in the literature that a P300 component in the EEG can be elicited by presenting visual stimuli according to an oddball paradigm [71]. More recently, it was demonstrated that also auditory [62] or tactile [12] oddball stimuli can produce evoked potentials in the EEG to be used for BCI. P300-based BCI systems 
have been applied for controlling wheelchairs [38], or spellers for paralyzed patients [68]. Because of the robustness of the approach, some commercial products are emerging, e.g. IntendiX [28]. Steady-state evoked potentials (SSEP) are another modality for BCI with the potential for clinical applications. These signals can be evoked by visual stimuli (steady-state visual evoked potentials, SSVEP) [63], or by tactile stimuli (steady-state somatosensory evoked potentials) [56], using a repetitive paradigm.

The above mentioned BCI methods are based on externally provided stimuli that the user receives passively while the BCI system aims at decoding their effects on the EEG activity. This type of BCI systems have complementary limitations and advantages. In general, the users are in the passive position when using these systems. On the other hand, evoked-potential systems need a specific intact sensory pathway, e.g. vision in visual P300, and are thus not applicable to patients with visual impairments.

A different BCI approach is based on decoding the volition of the user from EEG, for example by decoding motor tasks. Sensory-motor rhythms (SMR) [100] and movement-related cortical potentials (MRCP) [39] are examples of EEG signal components generated during voluntary motor execution (ME) or motor imagery (MI).

SMR corresponds to an increase or decrease in power at various subbands of EEG signals recorded over the sensory motor cortex (e.g., Cz for foot movements), prior to, during and after movement or movement imagination. The increase of subband power implies that the neurons in the corresponding 
cortical area discharge more synchronously than at baseline and therefore it is referred to as event related synchronization (ERS) [66]. Conversely, the decrease of subband power corresponds to less synchronous neural activity, termed event related desynchronization (ERD) [66].

MRCP is a type of slow cortical potentials [39], which appears as a negative variation in scalp EEG. Its magnitude starts to decrease, accompanying movements or imagination of movements, and reaches the negative peak around the movement or imagination onset, and afterwards it returns back to the baseline. For clarifying the terminology, it is worth mentioning that the $\mathrm{MRCP}$ is referred to contingent negative variation $(\mathrm{CNV})$ when generated in a synchronized (cue-based) paradigm [87], and Bereitschafts potential or readiness potential when generated in an asynchronized (self-paced) paradigm [39].

In the past decades, SMR has been the main signal modality used for this detection purpose. True positive rate (TPRs) above $80 \%$ were reported with Beta ERS [55][88], while the performance of ERD was also demostrated to be above $70 \%$ [70][99]. However, the detection latency was largely overlooked in these studies, with only one exception which reported a latency in the range of seconds [32]. On the contrary, due to its pre-movement feature, MRCP was demonstrated to be able to predict the motor intention [7], indicating a potential application in BCI. In this thesis, our aim is to develop self-paced, short-latency BCIs. Different from those systems based on evoked potentials, the patients would be put in the drivers place. Therefore, it is the user, 
rather than the computer, who will control the system. More importantly, the latency of our system would be short, or nearly real-time, which makes it effective for online applications in either communication or rehabilitation.

\subsection{Real-time brain-computer interface plat- form}

Aiming at developing self-paced, short-latency BCI systems, a real-time BCI platform was developed. It acquires and processes brain signals (EEG), as well as other inputs, in real-time. With its modular design, it is especially suitable for testing new algorithms in online studies. In addition, its extensible outputs are feasible for trigger external devices, such as electrical stimulator and robotics.

The real-time platform was built using Matlab Graphic User Interface (GUI)

for studies in Chapter 2, Chapter 3, and Chapter 4. It acquires multi-mode signals (e.g. EEG, EMG) from the hardware (see below), processing in realtime, and transform them into commands either for triggering external device or providing subjects feedback.

\subsubsection{Hardware}

A 16-channel g.USBamp amplifier (gTec GmbH, Austria) was used for collecting EEG signals from an actiCap electrode system (Brainproducts, Ger- 


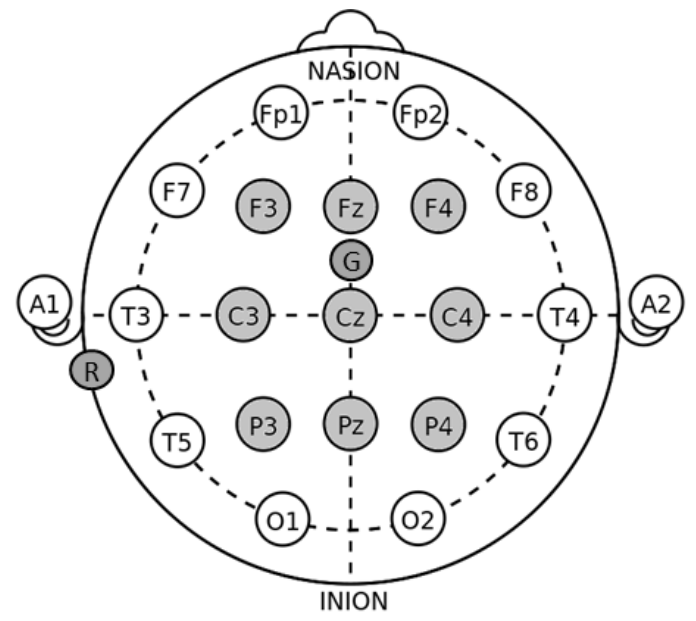

Figure 1.1: EEG channel locations

many). Nine channels of EEG was recorded from Cz, Fz, F3, F4, C3, C4, P3. $\mathrm{P} 4$ and $\mathrm{Pz}$ of the standard 10-20 locations, with the ground and reference on $\mathrm{AFz}$ and earlobe (or mastoid), respectively (see Figure 1.1). EMG was acquired using the 13th channel of the EEG amplifier. As we focus on the lower limb, a mono-polar EMG electrodes was placed on the bony surface of the Tibialis Anterior (TA) muscle, while its ground and reference electrode was put on the ankle and knee, respectively.

Through software settings, the nine EEG channels share their common ground and reference, while the EMG input has its separate ground and reference. A self-made pedal was used to fix the foot during experiment, and when necessary, the force of movement was recorded using a National Instrument card. 


\subsubsection{Software}

The software was developed based on Matlab GUI. The main body is the experimenter view (see 1.2), while subject view and output command may also be presented when necessary.

The most important part of the experimenter view is the control part in

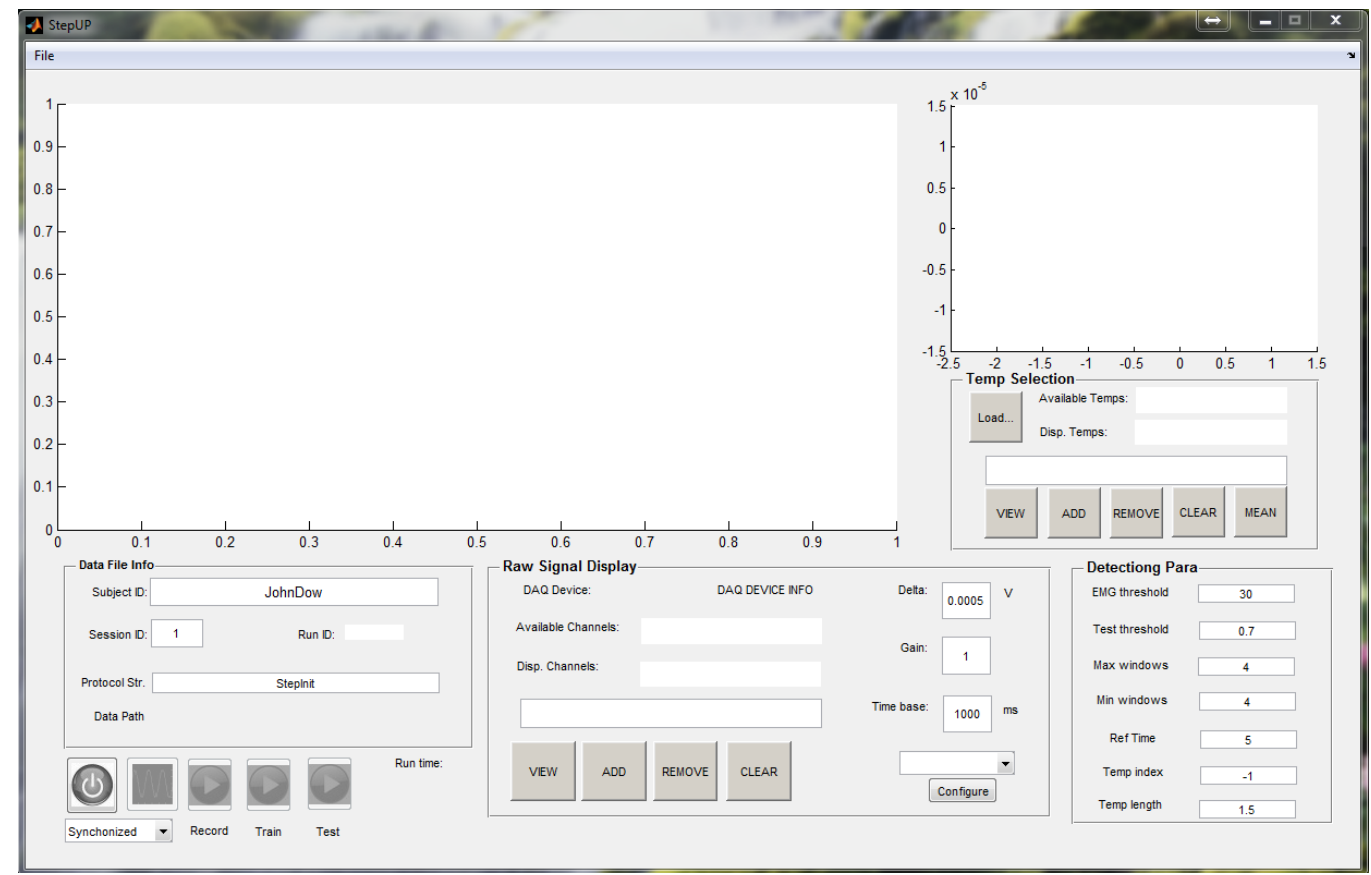

Figure 1.2: Experimenter View

the right bottom corner, which includes a selective menu and several control buttons. The selective menu has two options: synchronized or asynchonized, which correspond to synchronized (cue based) BCI paradigm or asynchronized (self-paced) BCI paradigm.

In the synchronized mode, there presents an additional subject view, in which 
a cue instructs the user to perform certain tasks. The prominent advantage of cue-based paradigm is that the timing of the tasks is recorded internally from the program, thus there is no need for external reference for the tasks (e.g.EMG). It is suitable to record motor imagery EEG, such as the comparison study in Section 2.2.

The self-paced paradigm is more frequently used in the following studies. It puts the user in the driver's place, and reflects the user's voluntary intention, which is basically the nature of a brain switch. Usually such a self-paced mode includes two sessions: a training session and a testing session. In the training session, the subject was asked to repeat some specific tasks (either self-paced or cue-based). The training data collected in this session was used to train a detector, which acts as the brain switch in the following testing session.

\subsection{Thesis structure}

The content of the thesis is organized as follows:

In Chapter 2, as the first step, the morphology of MRCP was investigated, with different external cues and motor tasks. Following that in Chapter 3, we developed a manifold learning method for real-time detection of MRCP, which realized a short-latency brain switch. Further, we studied three factors that may influence the performance of this brain switch. Based on these studies, two online BCI systems were implemented in Chapter 4, for appli- 
cation in stroke rehabilitation and communication, respectively. In Chapter 5, a pre-clinical study on spinal cord injured patients was performed, and the MRCP morphology in these patients was investigated . At the end, we summarize our work and discuss future directions in Chapter 6.

The thesis is based on the following conference and Journal papers:

1) R. Xu, N. Jiang, C. Lin, N. Mrachacz-Kersting, K. Dremstrup, D. Farina. Enhanced low-latency detection of motor intention from EEG for closed-loop brain-computer interface applications. IEEE Transactions on Biomedical Engineering, vol.61(2), pp.288-96, 2014.

2) R. Xu, N. Jiang, N. Mrachacz-Kersting, C. Lin, G. Asin, J. Moreno, J. Pons, K. Dremstrup, D. Farina. A closed-loop brain-computer interface triggering an active ankle-foot orthosis for inducing cortical neural plasticity. IEEE Transactions on Biomedical Engineering, vol.61(7), pp.2092-2101, 2014 .

3) R. Xu, N. Jiang, A. Vuckovic, M. Hasan, N. Mrachacz-Kersting, D. Allan, M. Fraser, B. Nasseroleslami, B. Conway, K. Dremstrup, D. Farina. Movement-related cortical potentials in paraplegic patients: abnormal patterns and considerations for BCI-rehabilitation. Frontiers in Neuroengineering, vol.7(35), 2014

4) R. Xu, N. Jiang, N. Mrachacz-Kersting, K. Dremstrup, D. Farina: Comparison of sensory-motor rhythm and movement related cortical potential during ballistic and repetitive motor imagery: an initial report. 3rd International Conference on Rehabilitation and Medical Engineering, 2014. 
5) H. Scheel, R. Xu*, N. Jiang, N. Mrachacz-Kersting, K. Dremstrup, D. Farina. Influence of external cues on synchronized brain-computer interface based on motor related cortical potentials. 7th IEEE EMBS Neural Engineering Conference, 2015 (*corresponding author).

6) R. Xu, N. Jiang, N. Mrachacz-Kersting, K. Dremstrup, D. Farina. Factors of influence on the performance of a short-latency non-invasive brain switch: evidence in healthy individuals and implication for motor function rehabilitation, Frontiers in Neuroscience, vol.9(527), 2015.

7) R. Xu, N. Jiang, S. Dosen, C. Lin, N. Mrachacz-Kersting, K. Dremstrup, D. Farina. Endogenous sensory discrimination and selection by a fast brain switch for a high transfer rate brain-computer interface IEEE Transactions on Neural System and Rehabilitation Engineering, 2016 (in press). 


\section{Chapter 2}

\section{MRCP morphology}

The morphology of MRCP may change according to the parameters of the movement, such as speed and force [19][20]. These changes may influence the performance of the detectors or classifiers [41], and thus lead to the instability of the BCI. In a synchronized BCI, a cue is usually used to indicate the timing of tasks, while different motor tasks may be used for inducing specific brain signal patterns. However, it is still unclear how these cues or motor tasks would affect the MRCP morphology. In this chapter, the impacts from the cue (visual or auditory) or motor task (ballistic or repetitive) were investigated respectively, and their potential influences on single-trial detection were discussed.

This Chapter is based on two conference papers [76][94], where I am the corresponding author and the first author, respectively. 


\subsection{The influence of the cue}

\subsubsection{Subjects and experiment}

Seven subjects (2 female, age: $22.7 \pm 2.2 \mathrm{yrs}$ ) participated in the experiment, in which they were instructed to perform ballistic dorsiflexions of the right foot according to a visual or an auditory cue. The visual cue was shown in the monitor in front of the subject, while the auditory cue was delivered via an earphone. In order to control the movement range and avoid fatigue, a bar indicating the force range of 20-40 \% maximum voluntary contraction (MVC) was also shown in the monitor. Additionally as a control, the subject was also instructed not to move or imagine but just follow the cues. These four trials, i.e. visual movement, auditory movement, visual no-movement, and auditory no-movement, were randomized in a block, so as to minimize the influence of sequence. Each trial started with a 6-s idle phase, followed by a preparation phase of $3 \mathrm{~s}$. For the visual cue, the preparation phase was indicated by a black cross on the screen, followed by a red arrow for the following task phase which lasted $1.25 \mathrm{~s}$ (see Figure 2.1). For the auditory paradigm, the preparation and task phase was indicated via the earphone with a single-beep and a double-beep sound, respectively (see Figure 2.1).

\subsubsection{Signal analysis}

Raw EEG signals were band-pass filtered at $0.05-3 \mathrm{~Hz}$ (2rd order butterworth), and then spatially filtered with a laplacian filter centered at $\mathrm{Cz}$, 


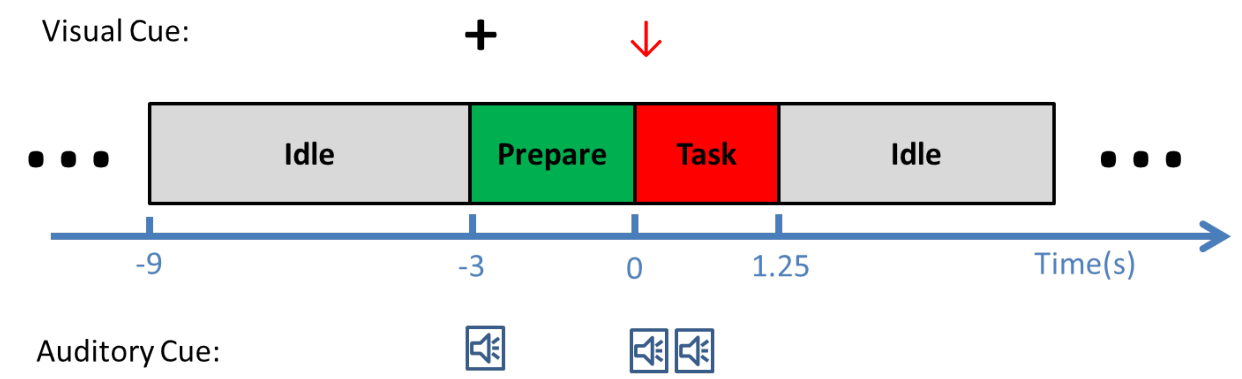

Figure 2.1: Visual and auditory cues. For a trial with either visual or auditory cue, it started with a 6-s idle phase. Following that was the preparation phase, indicated by a visual cross or single beep sound. The task phase in which the subjects performed the dorsiflexions was instructed with a red arrow or double beep sound

which resulted in a 'virtual $\mathrm{Cz}$ '. The MRCPs were segmented from -4s to 3s, with respect to the task onset, from raw $\mathrm{Cz}$ and virtual $\mathrm{Cz}$, respectively. Further, a paired t-test was performed to analyze the statistical difference across subjects between these two cues. The amplitude of MRCP, and the timing of its negative peak was compared in statistical analysis, and the significance level was set to 0.05 .

\subsubsection{Results}

The MRCPs from a typical subject are shown in Figure 2.2. It is clear that, for both raw and virtual $\mathrm{Cz}$, the auditory $\mathrm{MRCP}$ is larger in amplitude than the visual MRCP, while the negative peak of auditory MRCP was obviously longer delayed than that of the visual one. In addition, the amplitude of all signals were reduced after filtering. Especially for those with no-movement, 

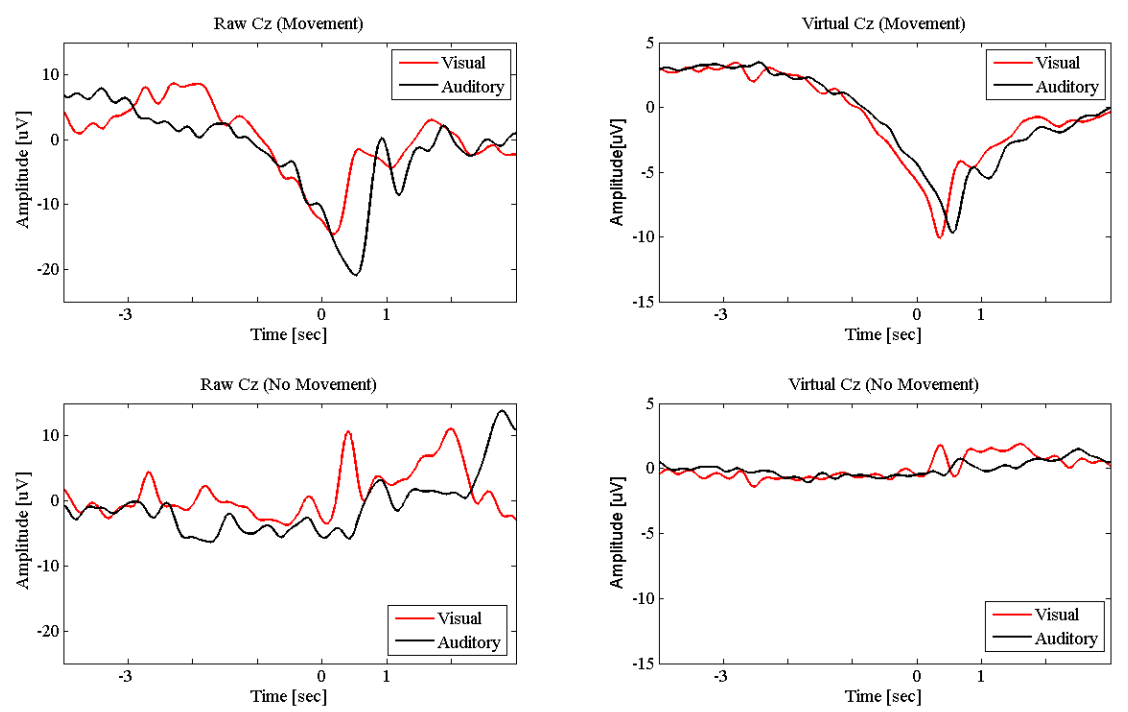

Figure 2.2: MRCPs with visual and auditory cues. The red and black lines stand for signals from visual and auditory trials, respectively.

the signal's amplitude after filter was almost $1 / 10$ of the original one. This demonstrated the ability of Laplacian filter in removing common noise and increasing signal-to-noise ratio.

The statistical analysis found that the difference in amplitude (visual vs auditory) was significant before filtering $(21.4 \pm 4.2 \mu \mathrm{V}$ vs $14.3 \pm 5.7 \mu V, p=0.008)$, while there was no significance afterward $(9.9 \pm 2.4 \mu V$ vs $9.4 \pm 1.5 \mu V, p=0.39)$, as shown in Figure 3.3. The negative peaks either before or after filtering were significant (Figure 3.4): $649 \pm 113 \mathrm{~ms}$ vs $488 \pm 232 \mathrm{~ms}(p=0.034)$, and $691 \pm 128 \mathrm{~ms}$ vs $414 \pm 54 \mathrm{~ms}(p<0.001)$, for raw and virtual $\mathrm{Cz}$, respectively.

The difference in amplitude may due to distinct origin of visual and auditory 


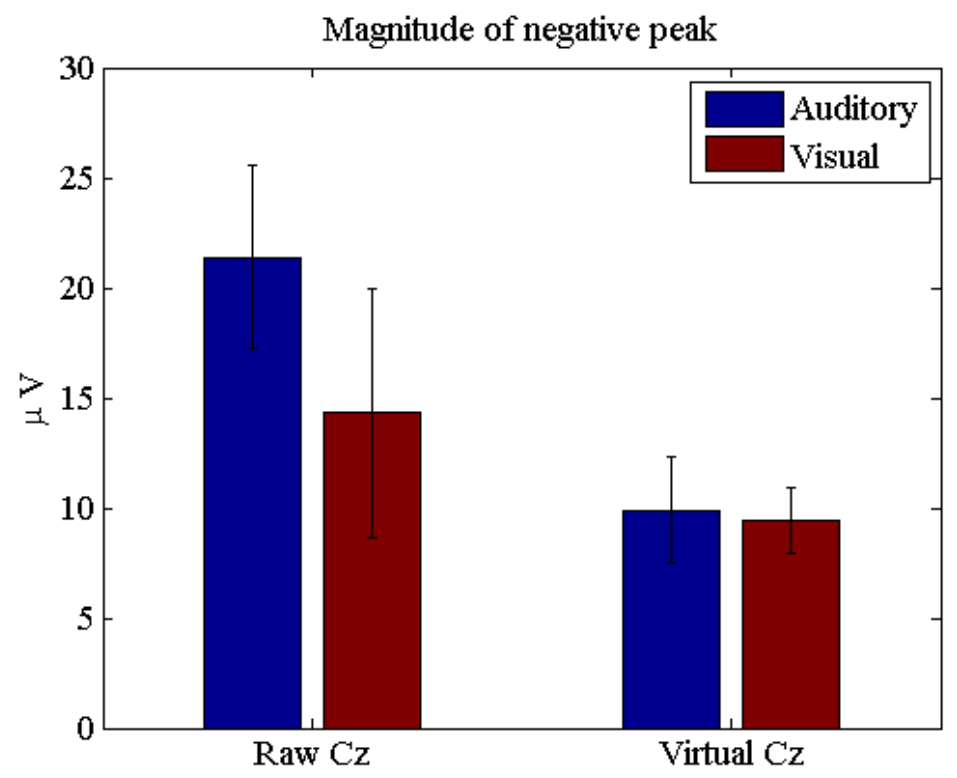

Figure 2.3: Magnitude of negative peak in MRCPs. MRCPs were from raw $\mathrm{Cz}$ and virtual $\mathrm{Cz}$, with auditory and visual cues, respectively.

input, which leads to a diverse impact on the motor cortex. The delayed negative peak of auditory MRCP may be mainly contributed from the long lasting cue (double-beep).We should acknowledge that the timing of the negative peaks may not exactly align, even in the same subject, due to the varied response time to the cue in each trial. This may also contribute to the difference in MRCPs between the two cues. 


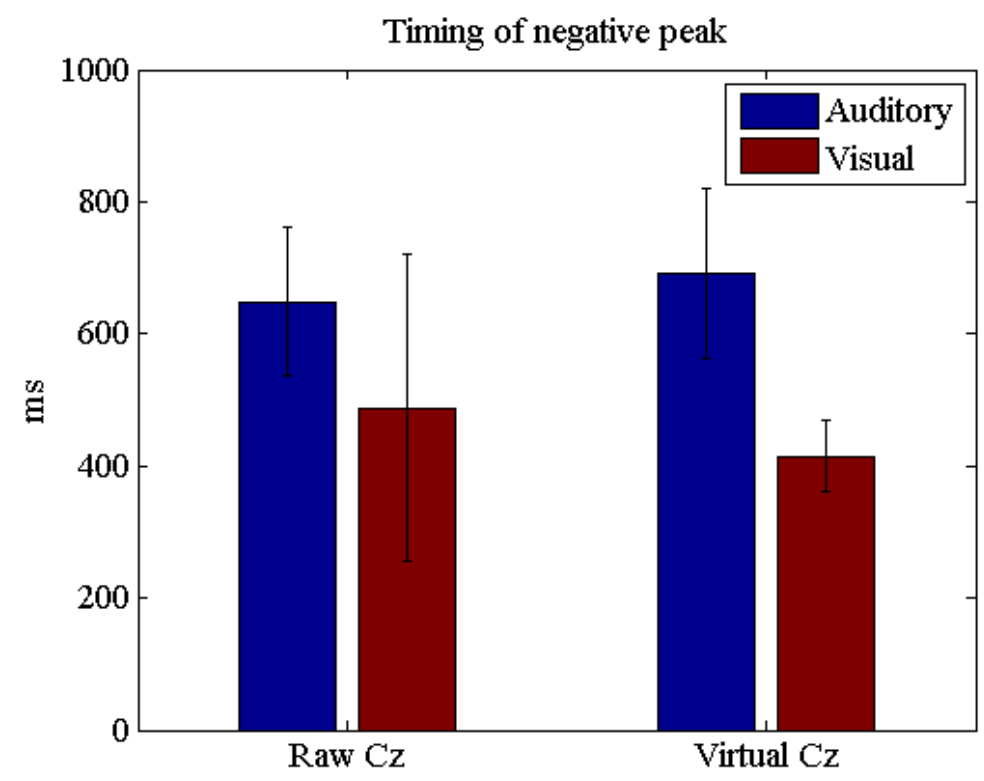

Figure 2.4: Timing of negative peak in MRCP

\subsection{The influence of the motor task}

In motor imagery based BCI, two types of motor task were frequently used. One is the ballistic task, which means the task was performed once and fast. The other task is the repetitive one, and the subjects were instructed to repeat the task for several times continuously. In this study, we recorded the EEG signals during these two motor tasks, and attempted to analyze their influence on MRCP morphology. 


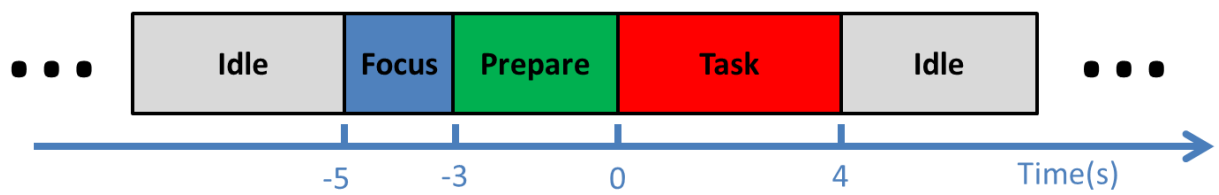

Figure 2.5: Experiment procedure with different motor tasks. Each trial began with an idle phase, followed by a 2 -s focus phase and a 3-s preparation phase. In the consequential task phase, the subject was instructed to perform ballistic or repetitive imagination of dorsiflexion.

\subsubsection{Experiment}

Ten healthy volunteers ( 3 female, age: $26.5 \pm 4.6$ yrs) participated in the study. During the experimental session, the subject was comfortably seated in a chair, approximately $1 \mathrm{~m}$ from a computer screen. Participants were instructed to look at the center of the screen and to follow the presented cue, minimizing eye movements. During the experiment, the cue on the screen indicated four states: idle, focus, preparation, and task. Each trial started from the 5-s idle phase, during which the subjects could adjust their position as they wished. In the second phase, the subjects were asked to focus on the screen without moving. This was followed by the preparation phase, where the subjects were instructed to follow the 3-s backwards counting presented on the screen, and to start imagining the movement immediately when it turned to the task phase, which lasted for $4 \mathrm{~s}$. One trial ended with the next idle phase before the next trial commenced.

Each experimental session was divided into six runs, which consisted of three 
ballistic and three repetitive runs. The ballistic and repetitive runs were identical, except in the task phase. For ballistic runs, the subjects were instructed to briefly imagine ballistic dorsiflexion, whereas they were continuously repeating motor imagery for the whole 4 -s task phase in repetitive runs. The TA muscle activity was monitored, and those trials with visible EMG signal, were not included in further analysis. Each run comprised approximately 20 trials of ballistic or repetitive imaginary movements. The duration of each run was approximately 6 min. The order of ballistic and repetitive runs was randomized.

\subsubsection{Comparison of MRCPs}

EEG signals were pre-processed as in Section 2.1.2, and in subsequent data segmentation, the data from $\mathrm{t}=-3 \mathrm{~s}$ to $\mathrm{t}=6 \mathrm{~s}$ of the filtered virtual $\mathrm{Cz}$ were extracted for each trial. The reference interval, from which the baseline value was calculated, was -3 s to -2 s (3 to 2 s before motor imagery onset).

A paired t-test was performed for MRCP morphology analysis. The comparison was between the amplitude of the MRCP for the reference interval (i.e. mean value of $-3 \mathrm{~s}$ to $-2 \mathrm{~s}$ w.r.t. task onset) and the magnitude of each 0.1s-length segments of the virtual $\mathrm{Cz}$ outside the reference interval. A Holm-Bonferroni correction was performed for this multiple comparison, and the significance level was set to 0.05 .

The MRCPs of a typical subject performing the two types of motor imageries are shown in Figure 2.6 (a) and (b). For the ballistic task (Figure 2.6 a), the 

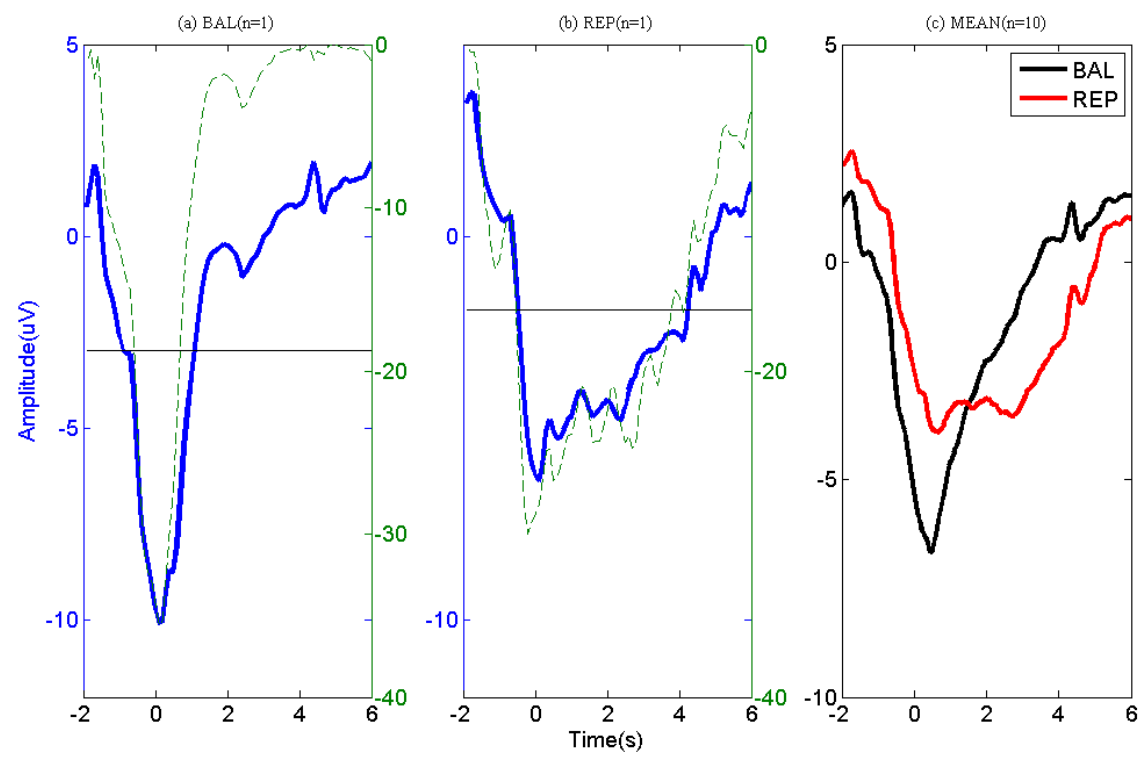

Figure 2.6: MRCP corresponding to (a) ballistic (BAL) and (b) repetitive (REP) motor imagery from a typical subject. The dashed lines indicate the logarithmic p-value of the paired t-test between the MRCP (either ballistic or repetitive) and its reference between -3 s and -2 s., while the solid horizontal line indicates significance level. (c): the average MRCP over the 10 subjects. The black line corresponds to ballistic imagery and the red line to the repetitive motor imagery.

MRCP started to decrease approximately $2 \mathrm{~s}$ before the task onset, reached the negative peak around $t=0 \mathrm{~s}$, and returned to the baseline in approximately 2 s. The repetitive MRCP (Figure $2.6 \mathrm{~b}$ ) had similar shape as for the ballistic case before $\mathrm{t}=0 \mathrm{~s}$ but the rebound phase was much longer in duration. Before task onset, both the ballistic and repetitive MRCP showed significant differences from the instant of -1 s. However, the situation of the two motor tasks was different for the rebound part. There was no significant 
difference for the ballistic MRCP from the time of $1 \mathrm{~s}$, indicating it already returned back to the baseline. On the other hand, the repetitive MRCP still showed significance as late as $4 \mathrm{~s}$ following peak negativity. The above difference between ballistic and repetitive tasks was consistent across subjects, as shown in Figure 2.6 c.

These above results indicate a strong predictive power of MRCPs in detection of movement intention of the subjects for both types of motor imageries.

\section{$2.3 \quad$ Summary}

In this chapter, the MRCP morphology under different conditions, i.e. the cue and motor task, were investigated. We found a larger amplitude and later negative peak of MRCP with the auditory cue than with the visual cue. The repetitive task resulted in a sustained negative phase in MRCP, while the MRCP returned back to baseline much faster in ballistic task. These difference may have direct impact on the single-trial detection of MRCP, and thus should be taken into consideration when designing MRCP based-BCI systems. 


\section{Chapter 3}

\section{Short-latency brain switch}

In the previous chapter, we investigated the factors that may influence the MRCP morphology, where the MRCPs were averaged from dozens of trials. For online BCI systems which desire accurate and fast decoding of brain signals, single-trials analysis of MRCP is required. However, due to the low signal-to-noise ration of EEG, as well as the large variability in MRCPs, the single-trials analysis is a challenging task.

In this chapter, we attempt to implement a short-latency brain switch by real-time detection of MRCP from single-trial EEG. Further, the key factors that may affect the performance of such a short-latency brain switch were investigated, so as to seek an optimal solution. Part of the materials used in this Chapter are from two first-authored journal papers: one has been published in IEEE [93], while the other in Frontiers [95]. 


\subsection{Real-time detection of MRCP}

For motor imagery based BCI, the motor intentions of the user need to be detected from brain signals with very short delay (several hundred ms), so that the resulting control can be perceived as a closed loop. For rehabilitation applications in particular, the afferents resulting from the peripheral stimulation triggered by the BCI detections have to reach the task specific cortical area within a very narrow time window so that the underlying Hebbian principle can be satisfied, and the rehabilitation can be effective [53]. For BCI based communication system, the performance is usually qualified by information transfer rate (ITR) [101]. The fast detection can reduce the time per trial, and thus increase ITR. Therefore, a short-latency brain switch can contribute to high performance BCIs for both rehabilitation and communication applications.

In a recent study by Niazi et al.[60], the MRCP was detected by a simple matched filter (MF), with supervised extraction of the MRCP template. This approach was a satisfactory balance between detection accuracy and latency [60] [58]. Furthermore, the MF is optimal for maximizing the signal-to-noise ratio when the noise is additive Gaussian. This condition is not necessarily valid in the current context. Thus, the MF is very likely to be suboptimal for this application. In the current study, a manifold learning algorithm, called Locality Preserving Projections (LPP) [33], followed by a linear discriminant analysis (LDA) classifier is proposed for the detection problem in 
MRCP-based BCIs. An offline pilot was first performed to tune the classifier parameter. Following that, an online BCI experiment was designed to compare the performance in detection accuracy and latency of the proposed algorithm with that of the MF approach.

\subsubsection{Locality preserving projection}

LPP is a manifold learning method for dimensional reduction, which preserves the local intrinsic structure of the data in the original high dimension. It has been successfully used in applications such as image processing [34]. The algorithm is presented as follows.

Given a graph $G$ with $N$ vertices, where each vertex represents a training sample, let $W$ be a $N \times N$ symmetric matrix with each $W_{i j}$ representing the distance between vertices $x_{i}$ and $x_{j}$, i.e., $W_{i j}=\exp ^{\left\|x_{i}-x_{j}\right\|^{2} / t}$. Here, $t$ is a constant. Then, $G$ and $W$ can be defined to characterize certain statistical or geometric property of the training samples.

Consider the problem of mapping samples on the graph $G$ to a line so that

the connected points are as close as possible. Let $y=\left[y_{1}, y_{2}, \ldots, y_{N}\right]^{T}$ be such a map. The optimal $y$ is given by

$$
\min \sum_{i, j}\left(y_{i}-y_{j}\right)^{2} W_{i j}
$$

Minimizing Equation 3.1 corresponds to ensuring that if the vertices $x_{i}$ and $x_{j}$ are 'close,' then the projections $y_{i}$ and $y_{j}$ are close as well [34], which explains 
the term 'locality preserving.' With algebraic formulations, we obtain

$$
\sum_{i, j}\left(y_{i}-y_{j}\right)^{2} W_{i j}=2 y^{T}(D-W) y=2 y^{T} L y
$$

where $D$ is a diagonal matrix whose entries are $D_{i i}=\sum_{j} W_{i j}$ and $L$ is the Laplacian Matrix. Finally, the minimization problem of Equation 3.1 equals to

$$
\min y^{T} L y \text { s.t. } y^{T} D y=1
$$

The constraint $y^{T} D y=1$ removes an arbitrary scaling factor in the embedding process. Note that $L=D-W$, so that Equation 3.3 equals to

$$
\max y^{T} W y \text { s.t. } y^{T} D y=1
$$

The optimal $y$ can be obtained by solving the following maximum eigen problem:

$$
W y=\lambda D y
$$

For classification purposes, a mapping for all samples, including new testing samples, is required. Suppose that $\alpha$ is a transformation vector, that is $y^{T}=\alpha^{T} X$, then the above eigenproblem can be transformed into

$$
X W X^{T} \alpha=\lambda X D X^{T} \alpha
$$


As illustrated in Figure 3.1, there are three steps for LPP [33][34]: first, the neighbors are selected to each sample of $X$ in the original high-dimensional space, after which the linear weights matrix $W$, i.e., the distance between each sample of $X$ and its neighbors, is calculated. Finally $X$ is projected to $y$, which is in the reduced-dimensional space.

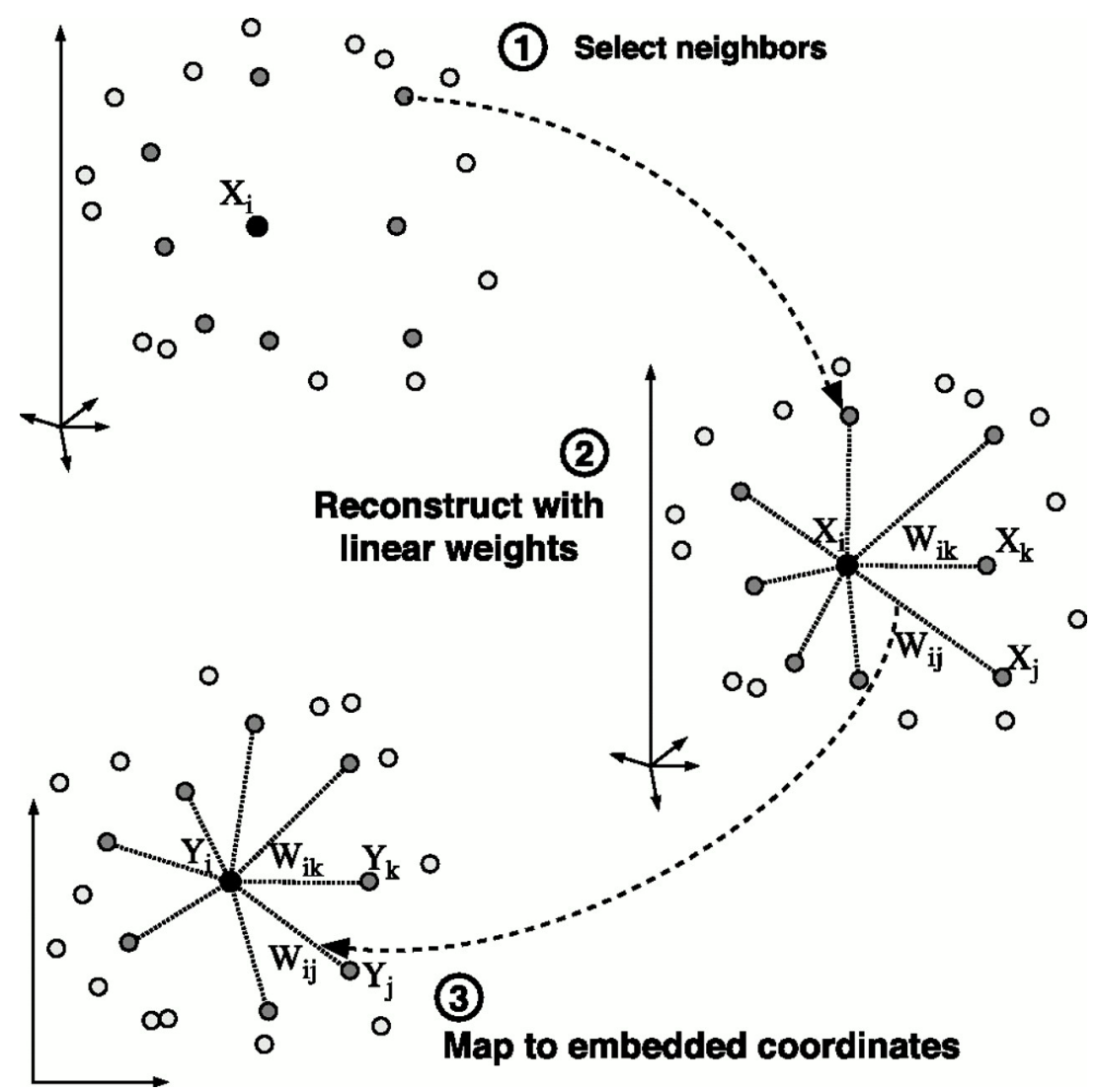

Figure 3.1: Three steps for LPP (adapted from [33], with permission to reproduce). Step 1: select neighbors for each sample, i.e. $X_{i}$ in original highdimensional space; step 2: reconstruct $X_{i}$ with linear weights $W_{i j}$, which represent the distance between $X_{i}$ and its neighbors, such as $X_{j}$; step 3 : project $X_{i}$ to the embedded coordinates, i.e. $Y_{i}$ in the reduced-dimensional space. 


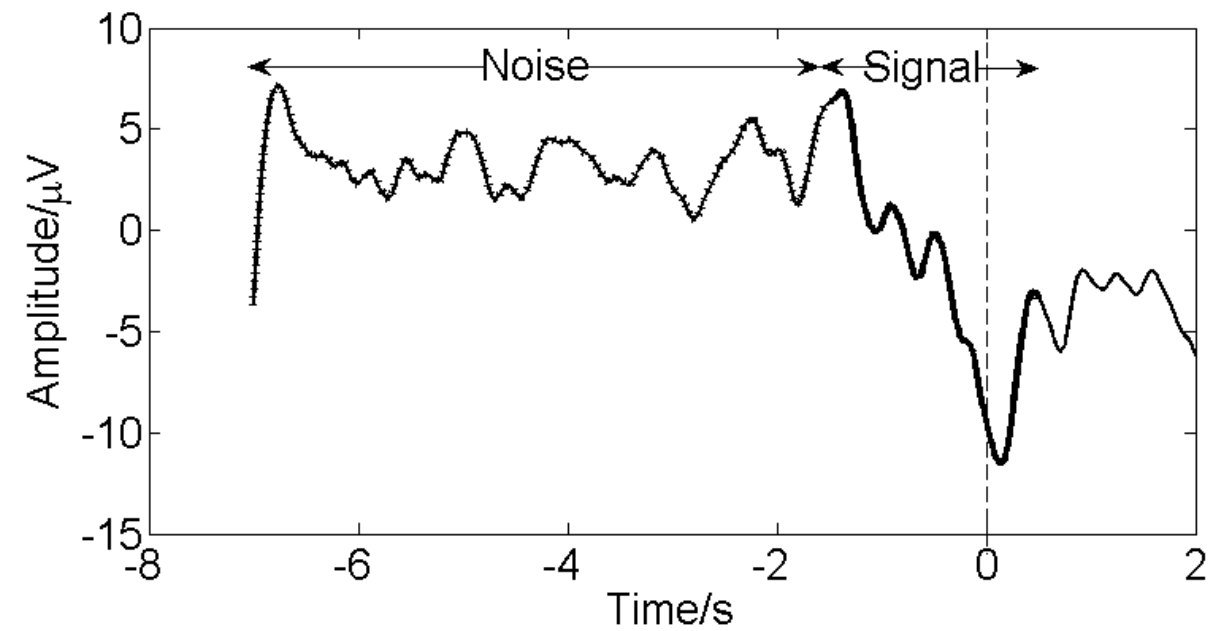

Figure 3.2: Single trial MRCP of self-paced dorsiflexion in training run $(n=1)$. Time zero is when the dorsiflexion was executed, as indicated by the dashed vertical line. For LPP training set, the signal from -1.5 to $0.5 \mathrm{~s}$ was taken as 'signal portion,' while others was taken as 'noise portion.'

\subsubsection{Offline parameter tuning}

For offline analysis, we investigated the effect of data dimensionality on the performance of LPP, and compared with a classic linear dimension reduction method: principal component analysis (PCA). The performance of LPP and PCA were also compared with that using the matched filter presented in [59]. PCA is a popular liner dimension reduction method. It projects the data (i.e. MRCP in this study) onto a lower dimensional coordinate system which kept the greatest variance (i.e. principal component). It has been frequently used in the signal processing field, such as EEG analysis [11], and thus the details are not repeated here. 


\section{Experiment}

A pilot experiment, in which four healthy subjects (age: $30.5 \pm 5.1$ yrs) participated, was used for the investigation on dimensionality. Subjects were instructed to perform ballistic ankle dorsiflexion movements following screen instructions (the same as in Figure 2.5), i.e. motor task execution in a synchronized mode. In each experimental session, two recording runs (continuous recording) were obtained. In each run, the subject performed 30 dorsiflexions, and the total time required for each run was on average seven minutes. Resting periods of approximately five minutes were provided between the two recording runs.

\section{Signal analysis}

The EMG signal was band pass-filtered from 10 to $500 \mathrm{~Hz}$. The TeagerKaiser energy operator was used to detect the onset of dorsiflexion as it was shown to be more accurate than using the amplitude of the surface EMG [46]. The timings of the onset detected from EMG were used as the reference timing for each dorsiflexion. The EEG signals were firstly band pass-filtered from $0.05 \mathrm{~Hz}$ to $3 \mathrm{~Hz}$, and then filtered by the Large Laplace Spatial Filter. The algorithms were trained or calibrated using data from the first run. Once they were trained, a pseudo-online processing approach, simulating online data streaming, was taken to process the data from the second run.

The EEG data between 1.5 second before and 0.5 second after each dorsi- 
flexion were extracted as the 'signal portion', while EEG data between 1.5 seconds before and 5 seconds after each dorsiflexion were extracted as the the 'noise portion' (Figure 3.2). For PCA-LDA, and LPP-LDA, MRCP detection is a two-class classification problem, where the 'signal portion' is one class, and the 'noise portion' is the other class.

The more conventional matched filter approach was also tested on the same data. For this approach, a MRCP template was extracted from the 'signal portion' of the training set, and detection threshold was selected from the receiver operating characteristic (ROC) curve obtained from the testing set.

Table 3.1: Offline performance

\begin{tabular}{clccccc}
\hline \multirow{4}{*}{ MF } & Subject & $\mathbf{1}$ & $\mathbf{2}$ & $\mathbf{3}$ & $\mathbf{4}$ & Mean \pm SD \\
& TP \% & 63.3 & 53.30 & 60.0 & 66.7 & $60.8 \pm 4.9$ \\
& FP/min & 1.4 & 4.1 & 3.3 & 2.7 & $2.9 \pm 1.0$ \\
& DL/ms & 229 & 58 & 263 & 694 & $311 \pm 234$ \\
\hline \multirow{3}{*}{ PCA } & TP \% & 76.7 & 86.7 & 90.0 & 83.3 & $84.2 \pm 4.9$ \\
& FP/min & 1.9 & 1.7 & 1.6 & 0.3 & $1.4 \pm 0.6$ \\
& DL/ms & 233 & 318 & 44 & 551 & $287 \pm 182$ \\
& Dimension & 73 & 95 & 57 & 93 & $80 \pm 16$ \\
\hline \multirow{2}{*}{ LPP } & TP $\%$ & 76.7 & 76.7 & 86.7 & 83.3 & $80.9 \pm 4.3$ \\
& FP/min & 2.3 & 2.7 & 0.6 & 1.3 & $1.7 \pm 0.8$ \\
& DL $/ \mathrm{ms}$ & 216 & 285 & 86 & 509 & $274 \pm 153$ \\
\hline
\end{tabular}

Summary of TP, FP, and DL of subject 1-4 for MF, PCA-LDA, and LPP-LDA. The optimal dimensions of PCA for each subject are also included.

\section{Results}

The performance of the two algorithms was calculated when the dimension 

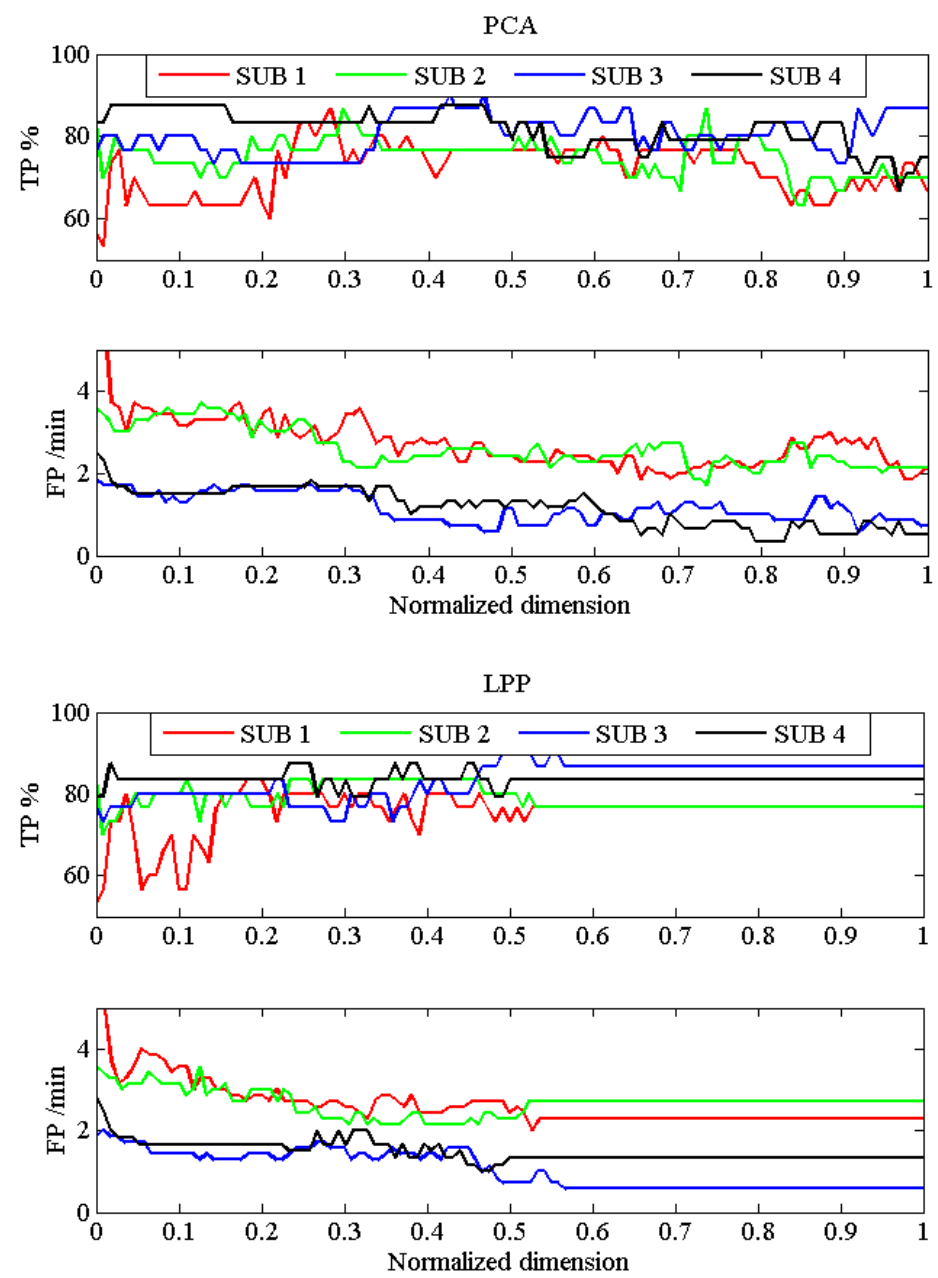

Figure 3.3: Offline performance with change of reduced dimension. PCA and LPP stand for the dimension reduction method of principal component analysis and locality preserving projection, respectively.

was varied from 1 to the rank of the covariance matrix of the training set. In order to qualify the dimension across different subjects, it was normalized with respect to the corresponding rank of the first run (i.e. the training run). Table 3.1 shows that the optimal dimension depended on the subject. 
The best true positive rate (TPR) was $84 \pm 5 \%$ and $81 \pm 4 \%$, with the false positive $(\mathrm{FP})$ of $1.4 \pm 0.6$ and $1.7 \pm 0.8$ per minute, for PCA and LPP, respectively. The detection latency for LPP and PCA was $287 \pm 182 \mathrm{~ms}$ and $274 \pm 153 \mathrm{~ms}$, respectively.

The important difference between the two methods was that, for LPP-LDA, both the TP and FP remained stable after the dimension exceeded 0.6 for all subjects, while no such trend could be observed from PCA-LDA (see Figure 3.3). This indicates that the optimal dimension of LPP-LDA can be set directly for all subjects. But the optimal dimension for PCA-LDA could only be obtained through a cumbersome search on a subject-by-subject basis, which is not desirable.

As shown in Table 3.1, PCA with optimal dimension yielded similar performance with LPP (TPR : $84.2 \pm 4.9 \%$ vs $80.9 \pm 4.3 \%$, FP: $1.7 \pm 0.8 / \mathrm{min}$ vs $1.4 \pm 0.6 / \mathrm{min}$, and DL: $274 \pm 153 \mathrm{~ms}$ vs $287 \pm 182 \mathrm{~ms})$. However, it requires a manually selected dimension, which would be a intractable issue for online BCI applications. On the contrary, LPP demonstrated its superior property with a subject-independent dimension, and thus was chosen for the following online study. Based on the above results of this pilot investigation, The optimal dimension of LPP was chosen at $60 \%$ of the original dimension, and applied to the online experiment.

\section{Representative detection output on the pilot data}

The performance of MF was also shown in Table 3.1. MF got a TPR of 60.8 
$\pm 4.9 \%, \mathrm{FP}$ of $2.9 \pm 1.0 / \mathrm{min}$ and $\mathrm{DL}$ of $311 \pm 234 \mathrm{~ms}$, which were clearly inferior to that of LPP. Figure 3.4 illustrated a representative example of the output of the two algorithms on the same training data in three-fold crossvalidation. LPP-LDA was capable of identifying true MRCPs yet rejecting non-MRCP signal segments with very similar morphology. In contrast, MF was shown to be easily influenced by small fluctuations, and missed true signals when the morphology changed.

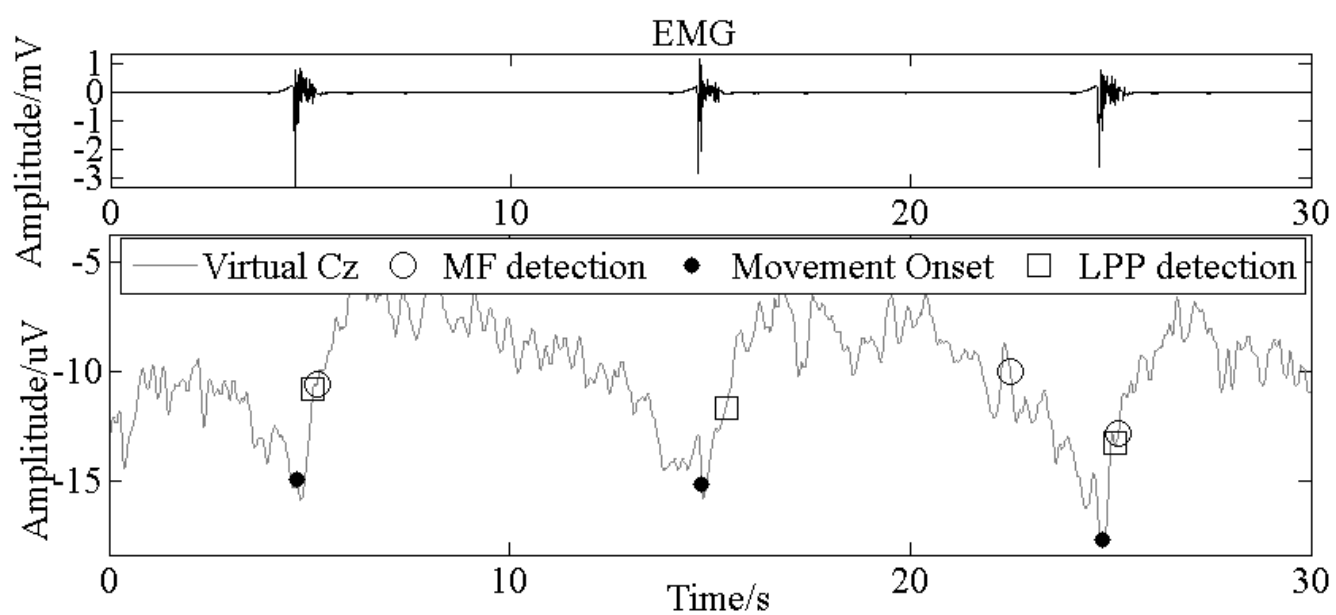

Figure 3.4: Representative detection output by MF and LPP-LDA on the same training data in three-fold cross validation. Filled cycles stand for the movement onsets detected from EMG; empty cycles are the MF detections, while squares are detections by LPP-LDA.

\subsubsection{Online experiment}

An online experiment was executed to test the validity of the proposed method since online performance is more relevant and closer to real-life ap- 
plications than offline analysis. The online experiment was executed on nine healthy subjects (age: $29.7 \pm 4.3 \mathrm{yrs)}$ and consisted of three sessions: preparation, training, and testing (see Figure 3.5).

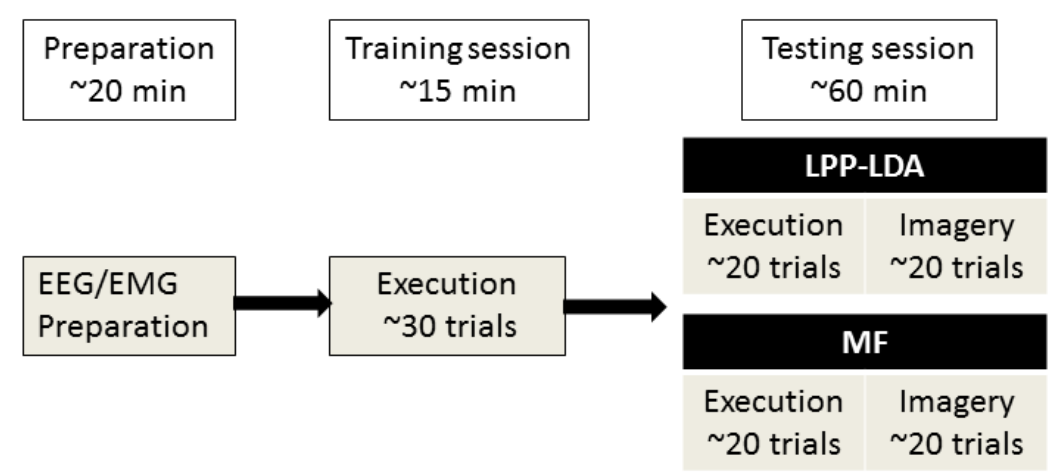

Figure 3.5: Online experiment flow. After preparation, the experiment started with a training session and a testing session in which self-paced ME and MI with LPP-LDA and MF were performed. In the last part the experiment, the order of the LPP-LDA runs and MF runs was randomized

\section{Session 1: preparation.}

In the preparation session, EEG and EMG electrodes were mounted according to the experimental setup described previously. The subjects were asked to perform ballistic dorsiflexions of their right ankle. The torque produced at the ankle joint was acquired and displayed in a subject-view GUI. In the beginning of each experiment, the subjects were instructed to perform a MVC for $10 \mathrm{~s}$. Then, the target contraction level in the training session was set between $40-60 \%$ of MVC. A vertical bar was displayed on the subject-view GUI for feedback purpose in the training session. The height of the bar was 
proportional to the torque value. The bar was green when the torque was within the target range and blue (red) when it was below (above) the target.

\section{Session 2: training.}

Once the target torque was set, the training session started. The subjects were asked to perform self-paced motor execution, i.e. real movements of dorsiflexions, with resting intervals of 10 - 20 s. Upon the detection of each movement from the EMG, an interval starting $2.5 \mathrm{~s}$ before and terminating $1.5 \mathrm{~s}$ after the movement onset of the preprocessed virtual $\mathrm{Cz}$ channel was displayed to the experimenter. Corrupted trials were flagged by the experimenter, and excluded from further processing.

There are two main reasons why real movements rather than cue-based imagery were used for the training set. First, the signal-to-noise ratio is higher for MRCP in real than in imagined movements [20]. Second, the MRCPs obtained from real movement can be aligned exactly by detecting the movement onset from the EMG, whereas there is an unpredictable alignment error for motor imagery. The use of real movements for training would be possible for patients who have residual muscle activity. Recently, high detection performance of voluntary movement onset was reported using EMG contaminated by spasm from stroke patients [102]. In our previous studies using MF on stroke patients, surface EMG from TA muscles was successfully used for the onset detection purpose [59] [52]. Cue-based imagery would be needed for patients without any residual activity, though. 
In the training session, the subjects were instructed to perform 30 successful executions. This number was chosen based on previous studies [59][60], as such reasonable length of repeated dorsiflexions would not make the TA muscle fatigue and thus the subjects could keep full concentration. It would be very difficult for stroke patient to stay alert and concentrate when the intervention protocol is too long. Because some executions were flagged as corrupted, the actual number of executions was usually slightly greater than 30. There were short rests upon the subjects requests. The data acquired in the training session were stored, and used to train the LPP-LDA classifier and the MF detector (see Section II-D).

For LPP-LDA classifier, there were five processing steps in the training stage of the signal processing (see Figure 3.6):

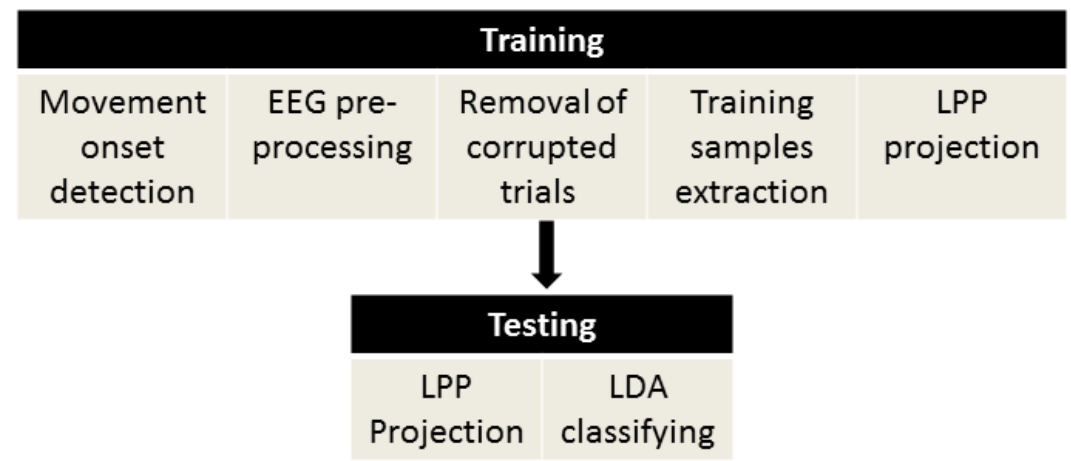

Figure 3.6: LPP-LDA classifier. The LPP-LDA classifier was trained offline using the data obtained from the training session, and then applied in the online testing session 
1) Movement onset detection

The Teager-Kaiser energy operator was used here to detect movement (selfpaced dorsiflexion) onsets from EMG online, which was shown to be more accurate than using the amplitude of the surface EMG [24].

2) EEG pre-processing

A band-pass filter from $0.05 \mathrm{~Hz}$ to $3 \mathrm{~Hz}$ and the Large Laplace Spatial Filter centered at $\mathrm{Cz}$ were used to enhance the signal-noise ratio of $\mathrm{Cz}$. This virtual $\mathrm{Cz}$ was used in subsequent processing steps.

3) Removal of corrupted trials

After the first two steps, $2.5 \mathrm{~s}$ before and $1.5 \mathrm{~s}$ after each movement onset of the pre-processed EEG, i.e. the virtual channel at $\mathrm{Cz}$, were presented to the experimenter immediately after each movement execution. Due to the subjects involuntary movement, such as eye blinking or jaw clutching during the training procedure, some trials were clearly corrupted. These trials were flagged by the experimenter, so that they could be excluded from subsequent processing.

4) Training samples extraction

The data between 1.5 second before and 0.5 second after the movement onset of virtual $\mathrm{Cz}$ were extracted as the signal portion, while the remaining EEG data were extracted as the noise portion (see Figure 3.2). Thus, the two-class training set X was compiled for the LPP-LDA classifier.

5) LPP-LDA classification

The LPP-LDA classifier was trained using the training set $X_{M \times N}$, where $\mathrm{N}$ 
is the number if training samples and $M$ is their original dimension. The training set $X_{M \times N}$, which includes both 'signal' and 'noise' samples, was projected to a lower dimensional feature space $y_{K \times N}^{T}$ (LPP space) using LPP transform vector $\alpha_{K \times M}^{T}$. K was the reduced dimension by LPP.

The MF training procedure was identical to LPP-LDA training from step 1 to step 4 (see II.D). MF only utilized the signal portion, from which an MRCP template was extracted. A three-fold cross-validation was applied to the training data, and the threshold of MF was chosen at the midpoint of the turning phase of the ROC curve [59].

\section{Session 3: testing.}

Once the classifiers were trained, the testing session started. Two runs (continuous recording) were performed in this session: LPP run and MF run, for the two detectors. In each run, the subjects performed first motor executions and then imagery. The order of the two runs was randomized. The execution tasks represents a test close to the real-life applications on patient with residual muscle activity while the imagery tasks represented the situation of patients who cannot execute any movement. Therefore, both of these tasks were carried out in the testing session and used to investigate the performance of the proposed method in detecting motor intentions from EEG.

In the LPP run, when the testing data stream (2-s windows with $0.1 \mathrm{~s}$ in-

crements) coming in, they were pre-processed as in the training session, and projected to LPP space using the same transform vector, and then LDA was 
used to classify them into 'signal' or 'noise'. When two consecutive decisions were classified as signal, an MRCP detection would be generated and a red bar in the subject-view GUI would be presented immediately.

In the online MF run, the incoming data was pre-processed, as done for the LPP-LDA. Then the likelihood ratio between the pre-processed incoming data and the templates was computed [59]. When two consecutive windows were above the MF threshold, a red bar in the subject-view GUI would be presented immediately, indicating an MRCP detection.

Both LPP-LDA and MF were applied on execution and imagery (see Figure 3.5). During execution, the subject was asked to perform self-paced dorsiflexions, as was done in the training session, for 15 - 20 times. During imagery, the subjects were asked to perform self-paced dorsiflexion imagery and to lightly say 'YES' $1 \mathrm{~s}$ after each imagery task in order to have a reference for the motor imagery events. In a preliminary investigation, it was determined that light simple speech did not influence the proper functioning of the two detectors. A red bar appeared in the subject-view GUI every time a detection occurred (either true or false detection). Both executions and imagery in the testing session were referred to as active phases. There was a passive phase before each active phase, in which the subjects were requested to totally relax for 1 min without any movement or motor imagery of the TA muscle. Normal eye movements, mouth movements, or slight trunk movement was allowed. This was to test the ability of the algorithms in rejecting potential artifacts produced by these factors under a realistic condition. In this phase, 
the number of passive false positive $(\mathrm{pFP})$ was counted for $1 \mathrm{~min}$.

During the testing session, the TPR, active false positive (aFP) and $\mathrm{pFP}$ were calculated online. The TPR was the ratio of true detections and total attempts, which were identified from the EMG channel for execution tasks and from subjects verbal indication for imagination tasks. The aFP was the number of false detection per minute in the active phases and $\mathrm{pFP}$ was the number of false detections per minute in the passive phases. Additionally, the detection latency (DL) was calculated offline. DL was the latency between the time of the detection and the EMG onset of corresponding movement for the executed tasks. For imagery, however, there was no EMG reference and thus the reference instant for latency calculation was the negative peak of the MRCP, which corresponds to the beginning of motor execution for executed tasks [41].

Two-way ANOVA tests were used to analyze significant differences among the performance of the BCI when varying the method for detection and the type of task. The response variable of the ANOVA tests included TPR, aFP, pFP, and DL. The two factors of the test were: tasks (two levels: execution and imagery) and algorithms (two levels: LPP-LDA and MF). For all tests, the full model was used first. When there was no significant interaction of the main factors, the reduced model with only the main effects was used. For all tests, the significance level was set to 0.05 . 


\subsubsection{Online performance}

The online performance, i.e. TPR, aFP, pFP and DL, were shown in Figure 3.7. The full model ANOVA showed that there were no significant interaction between algorithms and tasks for TPR, aFP, and pFP $(p=0.99,0.12$ and 0.27, respectively). Subsequently, reduced ANOVA tests with only main effects were performed. TPR with LPP-LDA was found to be significantly higher than that with MF (79 $\pm 12 \%$ versus $68 \pm 10 \%, p=0.001)$, and TPR of execution was also significantly higher than that of imagery $(78 \pm 11 \%$ versus $69 \pm 12 \%, p=0.015)$. Either aFP or pFP with LPP-LDA were found to be significantly lower ( $p=0.002$ and 0.022 , respectively) than those with $\mathrm{MF}(1.4 \pm 0.8 / \mathrm{min}$ versus $2.3 \pm 1.1 / \mathrm{min}$, and $2.1 \pm 1.9 / \mathrm{min}$ versus $3.5 \pm$ $1.3 /$ min respectively), while not significant with different tasks $(p=0.61$ and $p=0.10$, respectively). However, the full model ANOVA showed that there was significant interaction between algorithms and tasks for DL $(p=0.026)$. Subsequently, focused ANOVA tests were performed separately on the two factors. For execution, the DL with LPP-LDA was found to be significantly shorter than that with MF (235 $\pm 153 \mathrm{~ms}$ vs $462 \pm 147 \mathrm{~ms}, p=0.001)$, while for imagery, there was no significance in algorithms $(p=0.30)$. Also for LPPLDA, the DL of imagery was significantly longer than that of execution (396 $\pm 135 \mathrm{~ms}$ vs $235 \pm 153 \mathrm{~ms}, p=0.022$ ), while for $\mathrm{MF}$, there was no significance in tasks with $\mathrm{MF}(p=0.94)$. 


\subsubsection{Discussion}

In this study, a manifold based non-linear dimension reduction method (LPP) was applied, along with LDA, to perform online detection of MRCPs from EEG signals in execution and imagery of dorsiflexions. Across all subjects, the proposed algorithm had similar FP for execution and imagination and $\mathrm{TPR}>80 \%$ and $>70 \%$ for execution and imagery, respectively. These
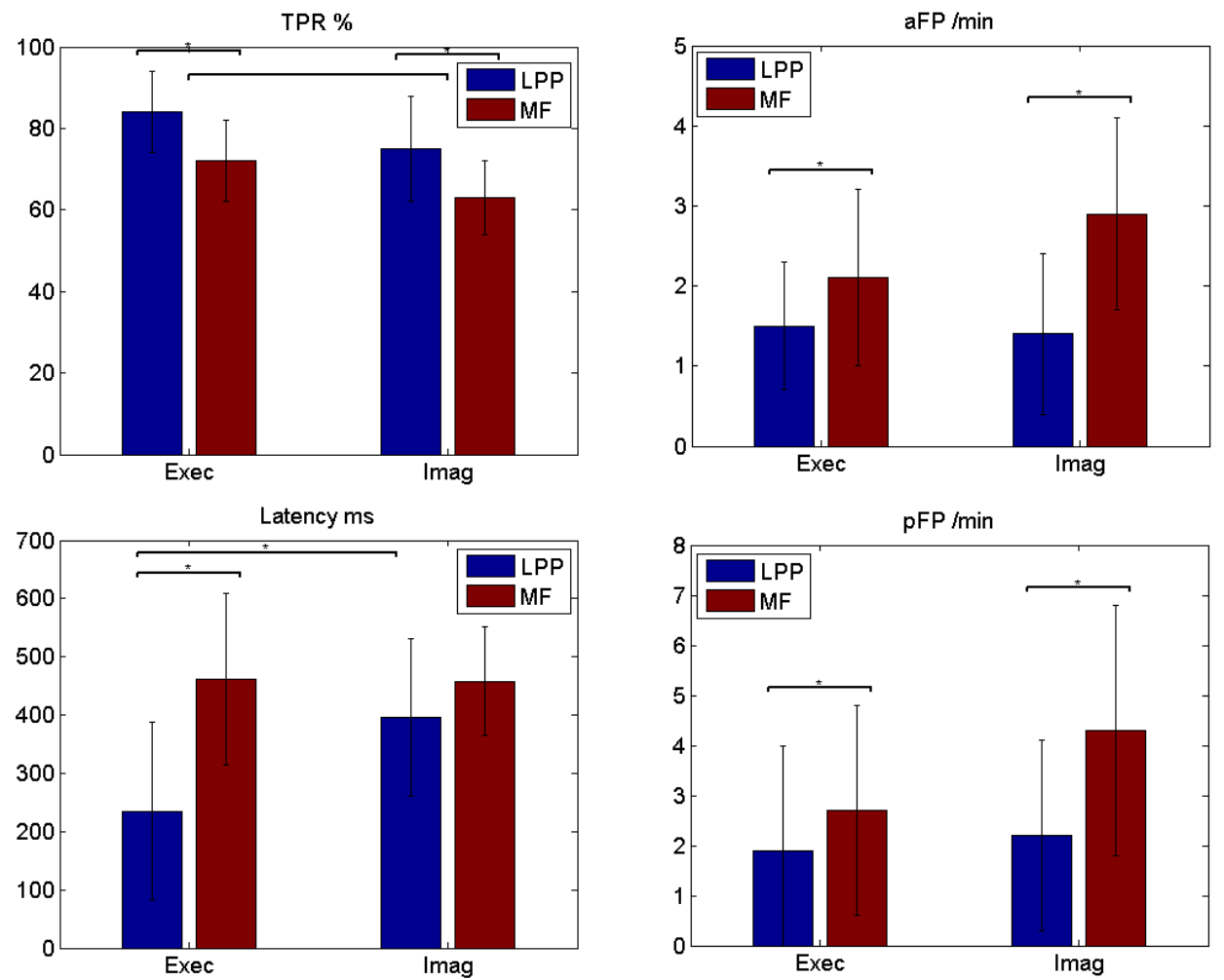

Figure 3.7: Online performance. TPR, aFP and pFP represent true positive rate, active false positive, and passive false positive respectively. The blue bars stand for the performance with locality preserving projection (LPP), while red bars are those with matched filter (MF). 
results were statistically better than those with the MF approach previously reported in the literature [59][60][58]. Further, the DL (219 $\pm 185 \mathrm{~ms})$ was also significantly shorter than that with MF in execution tasks.

\section{Robust performance}

The LPP-LDA yielded a significantly higher accuracy, compared with MF (see Table I). In addition, passive FP was tested in this study, and LPP-LDA also showed its superior ability in reducing $\mathrm{FP}$ when the subjects were in idle condition. More importantly, for LPP-LDA, there was no significant difference between the FP (both aFP and pFP) in both tasks. This insensitivity to task types demonstrated the robustness of LPP-LDA.

There are two reasons why LPP-LDA outperformed MF. One lies in the data organization. For MF, only the MRCP templates in the training session were used while the noise part was discarded. On the contrary, both signal portion (MRCP) and noise portion were used to train the LPP-LDA classifier, which thus made full use of the available information. Another reason is the powerful ability of LPP in preserving the intrinsic structure of the data in its original high dimensional space. That is why it could detect MRCPs when its morphology was changed, and reject false detections induced by signal fluctuation, while MF cannot (see Figure 3.4).

Moreover, LPP-LDA showed shorter latency than MF. In execution tasks, the DL with LPP-LDA was $\sim 200 \mathrm{~ms}$, which was significantly shorter than that with MF ( $400 \mathrm{~ms})$. In imagery tasks, the DL with LPP also tended 
to be shorter than that of MF, although no significance was found in this case, likely due to larger variability in the latency.

It should be noted that we used the signal of $0.5 \mathrm{~s}$ after the movement onset as part of the templates for both detectors, because the rebound phase (movement monitoring potential) is highly pronounced in many subjects. The DL could be further decreased by choosing a shorter portion of the MRCP as template, at the expense of detection accuracy.

\section{Subject independent parameters}

The threshold of MF output needs to be optimized. This step is usually

realized by a cross-validation of the training data on individual basis [59]. However, two issues should be treated carefully during this process. One is how to define the optimal parameters. There is a balance between the TPR and FP, but it is still not clear which one should have priority. Another issue is that the optimal parameter could be changed as the morphology of MRCP changes during different types of task (i.e., execution or imagery), and likely need to be calibrated from session to session. In the current study, even though the optimal parameter was chosen for the execution tasks, it may not be optimal for imagination tasks.

For LPP-LDA, no threshold needs to be set, and the only parameter that needs to be optimized is the dimension of the LPP space. As we demonstrated in Fig. 5, when the dimension was reduced to close to $60 \%$ of the total dimension of the training data, the performance of LPP-LDA kept con- 
sistently across subjects. This subject independent property is especially desirable for BCI applications.

In conclusion, we proposed a LPP-LDA classifier for detecting MRCPs in real-time, and compared its performance with the standard MF in an online experiment. We demonstrated that LPP-LDA performed significantly better than MF, with higher accuracy and lower latency. In addition, it showed the desirable property of subject-independent parameters. Based on these results, the proposed brain switch is an extremely promising tool for the development of user-intention-driven closed-loop BCI systems.

\subsection{Factors that influence the performance of the short-latency brain switch}

\subsubsection{Motivation}

In section 3.1, a shot-latency brain switch was proposed by real-time detection of MRCP using a manifold learning method called LPP. Specifically, MRCP was taken as the input signal, which is characterized by a very narrow frequency content $(0.05-3 \mathrm{~Hz})$ [93]. The same as other MRCP-based BCI studies, ballistic task, which means the real or imagined movement is executed once, was used for triggering motor task. Further, the time domain features were projected by LPP, and classified using a LDA classifier.

Apart from MRCP, several approaches have been proposed for motor inten- 
tion detection from EEG [85]. For example, SMRs have been intensively explored for the detection purpose [100].

In most SMR-based BCI studies for neurorehabilitation, the movement imagery that the subjects were instructed to perform was repetitive movement, such as foot tapping, with a few exceptions [67].

The above analysis indicates that a motor imagery based short-latency brain switch is predominantly influenced by three factors: the type of motor task (ballistic or repetitive), the frequency band (e.g. MRCP, Alpha or Beta band) of EEG, and the corresponding processing technique (subband power estimation or time-series analysis). In previous studies, the effect of some of these factors was partly investigated, e.g., SMR in brief and sustained movement [1][15] or MRCP vs SMR in finger movements [5][24][82]. More recently, there have been studies on the analyses of the frequency band in motor intention detection [27][37][48]. However, to date there has been no direct comparison of advantages and disadvantages of all the above factors in the context of short-latency brain switch for rehabilitation purpose. In this study on healthy subjects, these factors are directly compared in their influence on the detection of movement intention, attempting to provide a guideline for BCI researchers working toward neurorehabilitation applications.

\subsubsection{Methods}

The data were the same as those presented in Section 2.2. As the subjects and experiment procedure were described there, and thus not repeated here. 


\subsubsection{EEG processing algorithm}

\section{SMR morphology analysis}

Similar to the MRCP morpholoty analysis (see Section 2.2), the nine channels of EEG were band-pass filtered (2nd order Butterworth) at 4-40 Hz for SMR analysis [70]. Then a large Laplacian spatial filter centered at $\mathrm{Cz}$ was used to enhance the signal-noise-ratio of the virtual $\mathrm{Cz}$ channel, which was then processed in subsequent steps. In subsequent data segmentation, the data from $t=-3 \mathrm{~s}$ to $\mathrm{t}=6 \mathrm{~s}$ of the filtered virtual $\mathrm{Cz}$ were extracted for each trial. The reference interval, from which the baseline value was calculated, was $-3 \mathrm{~s}$ to $-2 \mathrm{~s}$ (3 to $2 \mathrm{~s}$ before motor imagery onset) for both SMR.

The power spectral density (PSD) of each trial was calculated over 1s windows overlapped for $0.5 \mathrm{~s}$ using Hamming windows (Matlab function pwelch). For each subject and each movement type, a Bootstrap test was performed between the PSD of the SMR at each time-frequency point and the reference PSD of the SMR in a baseline window. The time-frequency SMR characterization was quantified as follows:

$$
S M R_{f, t}=\left(A_{f, t}-R_{f}\right) / R_{f}
$$

where $S M R_{f, t}$ is the relative power of the SMR at time $t$ and frequency $f$, $A_{f, t}$ is the absolute power of the SMR at the same time-frequency point, and $R_{f}$ is the power of the reference interval for the frequency f. A positive $S M R_{f, t}$ value indicates an ERS, while a negative value of SMR indicates an 
ERD.

\section{Time-series feature extraction and motor imagery detection}

In order to analyze the information content in EEG for motor intention detection, six types of time-series features were extracted with band-pass filter at MRCP (0.05-3 Hz), Theta (4-7 Hz), Alpha (8-15 Hz), Beta (16-30 Hz), lower Gamma (31-40), and the full frequency band (0.05-40 Hz), followed by a large Laplacian filter centered at $\mathrm{Cz}$. In order to evaluate the BCI performance, a three-fold cross-validation was used, in which two runs of the virtual $\mathrm{Cz}$ (either ballistic or repetitive) were taken as the training set, while the remaining ballistic or repetitive run was used as the testing set. For both the training and testing set, the virtual $\mathrm{Cz}$ was segmented into portions of 2-s segments with 0.1-s increments. The segments between -1s to 1 s with respect to the task onset were labeled as signal portion, while all the remaining segments were labelled as noise segments. A 3-fold cross-validation classification was performed, as described in the following.

For the time-series feature, the proposed method, i.e. LPP-LDA, was used for motor imagery detection. The LPP-LDA classifier was trained using the training data and then used to classify the testing data into either 'signal' or 'noise'. A detection of motor intention would occur when a number of continuous windows (referred to window number (WN)) were classified as 'signal'. One detection would be determined either as a true or false detection according to the DL, i.e. the time difference between the detection and the 
task cue. If the DL was between $-1 \mathrm{~s}$ and $1 \mathrm{~s}$, the detection was considered a true detection, otherwise a false detection. It should be emphasize here that the target of this study is a short-latency brain swtich. Therefore we only considered these detections within a few hundreds ms, even though the later part (e.g. Beta rebound) may improve the accuracy. TPR in percentage, FP per minute, and DL were calculated to quantify the BCI performance. There is a trade-off between TPR and FP, depending on the WN. Both the TPR and FP decrease with an increase in WN. In order to objectively compare the BCI performance across these factors, we chose for all comparisons the WN value for which the FP was $\leq 8 / \mathrm{min}$. Thus only the TPR and DL were statistically compared in the following section.

\section{Subband power estimation and motor imagery detection}

The subband power was also used as direct feature for classification. For this purpose, the powers of the virtual $\mathrm{Cz}$ channel at the six frequency bands were estimated using the Welch periodogram with a resolution of $1 \mathrm{~Hz}$. As

for the time-series features, the window duration was of $2 \mathrm{~s}$, with increments of $0.1 \mathrm{~s}$. The 'signal' and 'noise' portions were the same as for the time-series features, and the same three-fold cross-validation method was used to test performance.

As the dimensions of the subband power features are small, no dimension reduction method was used. The signal and noise portions were directly used to train a LDA classifier, which was used for detection of motor intention from 
the testing set. All BCI performance criteria were calculated with the same steps as in the time-domain processing, and analyzed using the statistical methods described in the following.

\subsubsection{Statistical methods}

Three-way repeated ANOVA was used to investigate the effect of the three factors on BCI performance. The independent variables were TPR and DL. The three main factors were motor task (ballistic and repetitive imagination), frequency band (MRCP, Theta, Alpha, Beta, Gamma, and full frequency bands), and processing technique (time-series analysis and subband power estimation). A full model ANOVA with all interaction terms was performed first and, when significant interactions were detected, post-hoc tests (Tukey simultaneous test with significance level of 0.05) were performed.

\subsubsection{Results}

\subsubsection{Signal Morphology}

\section{MRCP morphology}

The morphology of MRCP was shown already shown in Figure 2.6, where we found its consistent feature cross subjects that the repetitive MRCP has a sustained negative phase.

\section{SMR mapping}



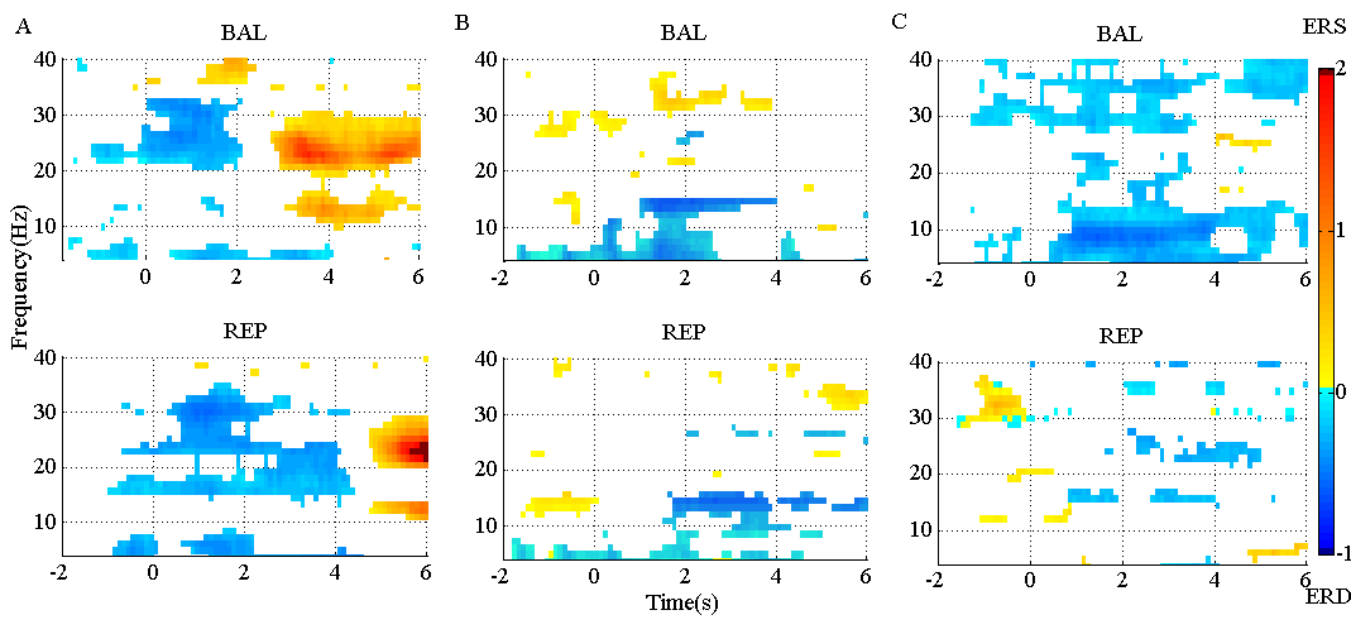

Figure 3.8: SMR mapping from three representative subjects. BAL and REP stand for ballistic and repetitive task, respectively. Only those points with significance in bootstrap test are presented. Red area indicates ERS, while blue area is ERD.

The SMR maps for three representative subjects for the two types of movement imageries are shown in Figure 3.8. For subject A, there was a evident ERD starting slightly earlier than the task onset, between the Beta and the lower Gamma band (above $\sim 20 \mathrm{~Hz}$ ) for the ballistic imagery, while it corresponded to a larger bandwidth for repetitive imagery. There was also a evident ERS in the Alpha and Beta bands for both tasks, but the repetitive ERS occurred much later than the ballistic one. The situation was very different for subject B, whose ERD and ERS mainly appeared in the Alpha and lower Gamma band, respectively. Subject C showed still other characteristics. The ERD occurred over almost the full band for the ballistic imagery, whereas it did not present a clear pattern in the repetitive imagery. 
Moreover, for all subjects, both the ERD and ERS occurred earlier in case of ballistic imagery with respect to repetitive imagery. ERS appeared before imagery onset in both ballistic and repetitive tasks of subject B, and also in the repetitive task of subject $\mathrm{C}$.

In summary, the SMR mapping differed substantially among the subjects, thus a general average across the subjects is non-meaningful and therefore not presented here.

\subsubsection{BCI performance}

The BCI performance in detection of motor imagery is summarized in Table

3.2. The ballistic MRCP with time-series analysis reached the highest TPR

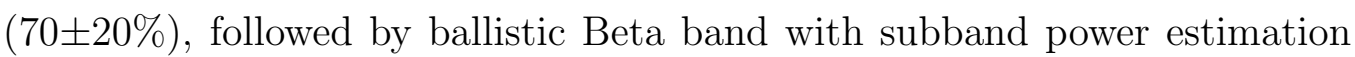
(57 $\pm 19 \%)$. SMRs with time-series analysis yielded shorter DL $(<100 \mathrm{~ms})$, however the corresponding detection accuracy was extremely low $(\sim 30 \%)$. For each frequency band of either motor task (except the full band of ballistic task), the time-domain technique resulted in shorter DL than the frequencydomain technique.

\section{Analysis of True Positive Rate}

For a meaningful comparison between methods, the TPR is reported in all cases for the same level of false positive $\left(\leq 8 \mathrm{~min}^{-1}\right)$. The three-way ANOVA on TPR found no three-way interaction $(p=0.074)$, and also no interac-

tion between motor task and processing technique $(p=0.283)$. Conversely, 
there was a significant interaction between motor task and frequency band ( $p=0.015)$, as well as between processing technique and frequency band ( $p<$ 0.001). Therefore, we performed post-hoc tests on the significant interactions.

Focusing on the interaction between motor task and frequency band, the post-hoc comparison found that MRCP with ballistic task yielded the highest TPR $(60 \pm 22 \%)$. This was comparable to the full band with the same task, and significantly better than all other combinations (no significance was found among them). In addition, for both MRCP and full band, ballistic task significantly outperformed repetitive one.

For the other significant interaction between processing technique and frequency band, the difference depended on each factor. MRCP with time-series analysis provided the highest TPR $(57 \pm 22 \%)$, which was significantly better than all other frequency bands with the same processing technique. For the other processing technique, i.e. subband power analysis, there was no significant difference among frequency bands. Furthermore, it was observed that, for Alpha and Beta band, subband power analysis significantly outperformed time-series analysis. 


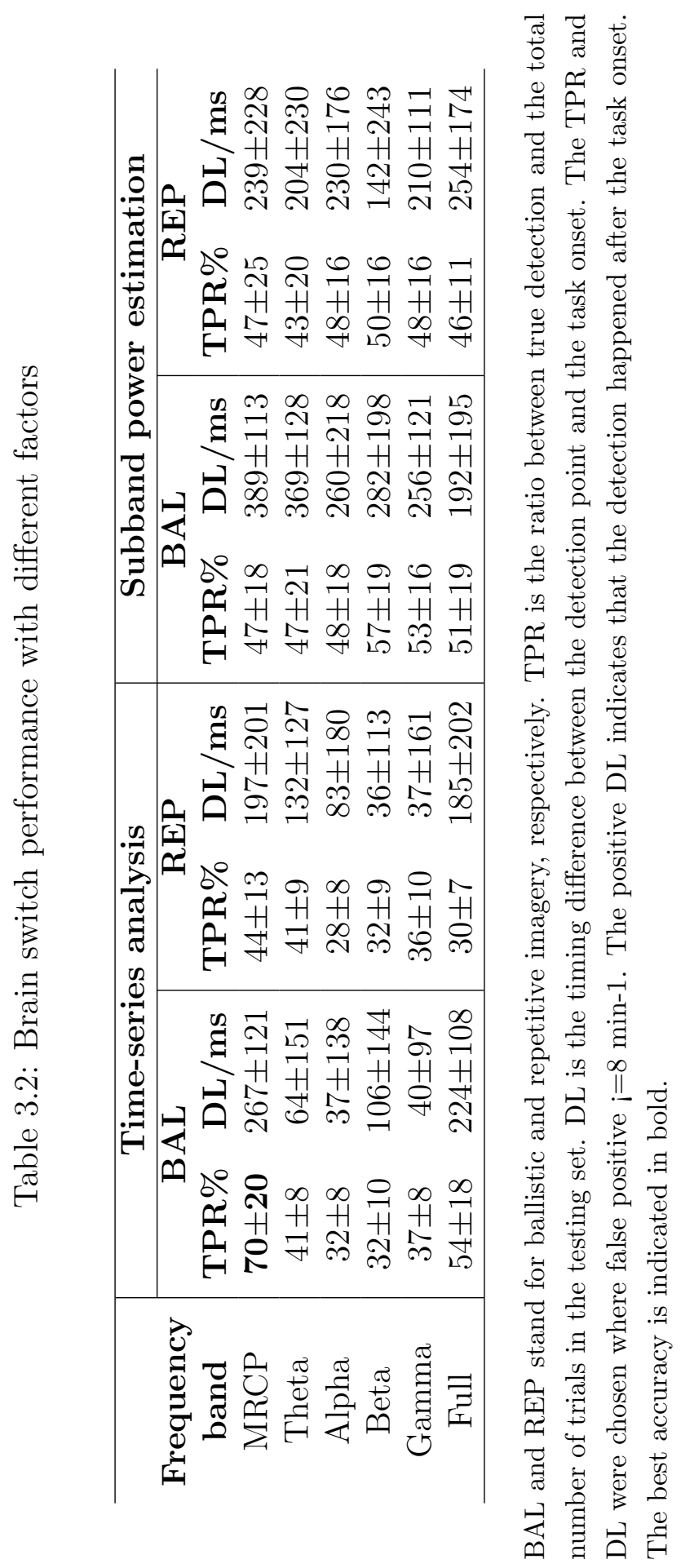




\section{Analysis of detection delay}

There was no three-factor interaction $(p=0.451)$, nor two-factor interactions ( $p=0.197,0.532,0.081)$. Both the processing technique and frequency band were significantly different $(p<0.001$ and $p=0.004$, respectively). The posthoc comparison revealed that the time-domain technique had lower DL than the frequency-domain technique (117 $\pm 169 \mathrm{~ms}$ vs $252 \pm 194 \mathrm{~ms})$, while the MRCP resulted in longer DL than Alpha, Beta and Gamma band (265 185 ms vs $145 \pm 205 \mathrm{~ms}, 129 \pm 193 \mathrm{~ms}$ and $125 \pm 159 \mathrm{~ms}$, respectively).

\section{Summary}

Based on the above statistical analysis of TPR and DL, we summarize the influence of the three factors here. The ballistic task, which yielded higher TPR for both MRCP and full band, is preferable over the repetitive one. Even though MRCPs DL was slightly larger than for some SMRs, MRCP was still the best choice among all six frequency bands, given its highest TPR with either ballistic task or time-series processing technique. Time-series analysis outperformed subband power analysis, mainly due to a significantly shorter DL.

In summary, this comprehensive comparison on motor intention detection with two motor tasks (ballistic and repetitive), six frequency bands (MRCP, Theta, Alpha, Beta, Gamma and full band), and two processing techniques (time-series analysis and subband power estimation) showed that, in general, the combination of ballistic, MRCP and time-series analysis significantly out- 
performed the other options.

\subsubsection{Discussion}

The challenge of detecting the motor intention from scalp EEG is an active topic in motor imagery based BCI. Recently, clinical studies using SMRbased BCI are being reported [73]. Moreover, MRCPs have also been proven as promising signal type, particularly because of the short detection latency [60][93]. Preliminary studies of MRCP-based BCI in chronic stroke patients have been reported, showing significant success as exemplified by functional improvements in the target population following a very short intervention $[54]$.

According to the Hebbian principle [35], neuroplasticity would only be induced when the motor intention and the task specific afferent feedback occur synchronously. Therefore, such kind of effective neuromodulation system requires a fast and accurate detection algorithm.

In the past decades, SMR has been the main signal modality used for this detection purpose. TPR above $80 \%$ was reported with Beta ERS [55][88], while the performance of ERD was also shown above $70 \%$ [70][99]. However, the detection latency was largely overlooked in these studies, with only one exception which reported a latency in the range of seconds [32]. On the other hand, slow cortical potentials such as MRCP have been investigated for movement intention detection in recent years [6][45]. In the current study, the average TPR and DL was $70 \%$ and $<300 \mathrm{~ms}$ for motor imagination, 
consistent with the results reported in previous studies [59][60][93][96]. The relatively good performance both in $\mathrm{TPR}(>70 \%)$ and $\mathrm{DL}(<300 \mathrm{~ms})$ is instrumental for the high efficiency of cortical plasticity induction of MRCPbased BCI system. In particular, the detection latency of a few hundred ms was demonstrated to be crucial for plasticity induction [53].

In this study, we found that ballistic motor imagery task, frequency band of MRCP, and time-series analysis is the optimal combination in terms of detection accuracy. Other options are sub-optimal mainly due to a trade-off between TPR and DL.

Repetitive SMRs with subband power estimation was found to be significantly better in accuracy than the ballistic one, which is in accordance with the discussion by Pfurtscheller \& Solis-Escalante that SMR in repetitive task is easier to classify [65].

The morphological difference between ballistic and repetitive tasks may mainly contribute to the detection performance of the two motor tasks. The prolonged negative phase in repetitive MRCP (in Figure 2.6) makes its rebound feature after task onset not as distinct as the ballistic one. That is why the accuracy of repetitive MRCP with time-series analysis is much lower than the ballistic one. On the other hand, in the case of subband power analysis, the frequency band did not have a significant influence on accuracy, as there is difference large variability in the optimal frequency bands among individual subjects (see Figure 3.8). 


\subsection{Summary}

In this chapter, we proposed a short-latency brain switch by real-time detection of MRCP from self-paced motor imagery. A manifold learning method called LPP, followed by a LDA classifier was used for this detection purpose. Its robust performance was demonstrated in an online experiment, with a TPR of $\sim 80 \%$ and FP of less than 2 per minutes. More importantly, it yielded a DL of $\sim 300 \mathrm{~ms}$, indicating a nearly real-time detection. These results were superior to classic methods, such as PCA and MF. Further, we investigated the factors of influence on the short-latency brain switch. The detection performance was compared among motor tasks, frequency bands and processing techniques, resulting an optimal solution with MRCP from ballistic task using time series analysis. Based on these work, we are able to develop totally self-pace, real-time closed-loop BCI systems. 


\section{Chapter 4}

\section{Closed-loop BCI applications}

The aim of this chapter is to develop closed-loop applications of the shortlatency brain switch proposed in Chapter 3. The first application is a mechanical stimulation system driven by the brain switch, aiming at stroke rehabilitation. The second one is a BCI communication system, in which the brain switch triggers a selective eletro-tactile menu.

The figures and text in this Chapter are partially from two journal papers where I am the first author. One paper has been published in IEEE [96], while another one is in press [92]. 


\subsection{Brain switch driven mechanical stimula- tion for rehabilitation}

\subsubsection{Motivation}

Motor disorder caused by central neural system (CNS) injury, e.g. stroke, is one of the main healthy problems worldwide. According to the statistic from World Health Organization, there are around 15 million people suffering stroke each year [91]. This number tends to increase due to several reasons, such as changing of diet structure and life style, and the aging population. On the other hand, the acute fatality rate following CNS injury has been decreasing with the improvement in medical treatment. This leads to an fast increasing population who urgently need proper rehabilitation.

Traditional rehabilitation, i.e. therapist centered approach, is currently the main treatment for these patients. However, in that process, the patients are moved passively by the therapist, receiving fairly primitive feedback, which usually results in a long-lasting period of rehabilitation without efficient progress.

Recently, BCI systems have been developing as a promising rehabilitation tool for neurological disorders, such as stroke [2][4][73][54]. Compared with the traditional therapy, the notable advantage of BCI rehabilitation system is that the patient was put in the driver's place who participated in the rehabilitation process in an initiative way. Specifically, in this kind of application, 
$\mathrm{BCI}$ is used to detect motor intentions of the user during motor imagination, triggering electrical stimulation (ES) [54], or visual feedback [73], in order to re-establish a closed-loop control.

One key issue for such kind BCI application to be effective is the precise timing between the motor intention and associated afferent feedback. This timing is critical for learning, as has been demonstrated by Hebb who proposed that only if the pre and post-synaptic cells are activated simultaneously, will the synapse be strengthened [35]. Numerous studies have verified this concept in animal preparations where long term potentiation (LTP), i.e. the long lasting increase in synaptic efficacy, has been proposed to be the primary mechanism for memory retention and learning. For a BCI to be implemented within the context of neuromodulation, it is thus imperative that the afferent feedback is timed to arrive in synchrony with the imagined movement [53]. Therefore, the short-latency brain switch, as demonstrated in Chapter 3, would be especially suitable for such application.

Proper sensory feedback is another key factor for effective BCI based rehabilitation. In previous study, the ES at sub-motor threshold was used for sensory input [60]. This may be replaced by other stimulation modalities that could provide more natural sensory feedback. Presumably, more natural afferent feedback would be more effective in plasticity induction.

In the current study, we apply the robust short-latency brain switch to trigger a Motorized Ankle-Foot Orthosis (MAFO), which generates more natural afferent feedback. The resulting system is extensively tested in the current 
study on healthy subjects to provide the first evidence of plasticity induced by triggering an active orthosis with precise temporal association with motor imagination detected by MRCP.

\subsubsection{System development}

The brain-computer interface driven motorized ankle-foot orthosis (BCIMAFO) system includes two main components: a brain switch which detects motor intention from EEG, and a mechanical stimulator which executes the intention with the passive dorsiflexion, delivering sensory feedback to the brain (see Figure 4.1). As the brain switch was detailedly described in last chapter, it is not repeated here.

The MAFO is a wearable ankle foot exoskeleton, designed specifically for ambulatory rehabilitation of drop-foot [72]. It provides natural approach for inducing sensory feedback by mimicking the real movement. It is a single degree-of-freedom (ankle dorsiflexion and plantarflexion) mechatronic device, which integrates state-of-the-art planar DC motors and planar harmonic transmissions for a compact and light system design. A Matlab Real-Time Workshop system (Mathworks Co.) was used as the real-time control interface of the MAFO. This allows easy interfacing with other equipment for bio-signal acquisition and processing. MAFO has been subject to extensive electromechanical testing which renders it suitable for clinical research. Various control modes can be implemented in the control architecture of MAFO, in particular it can block the joint for isometric states, and impose isokinetic 


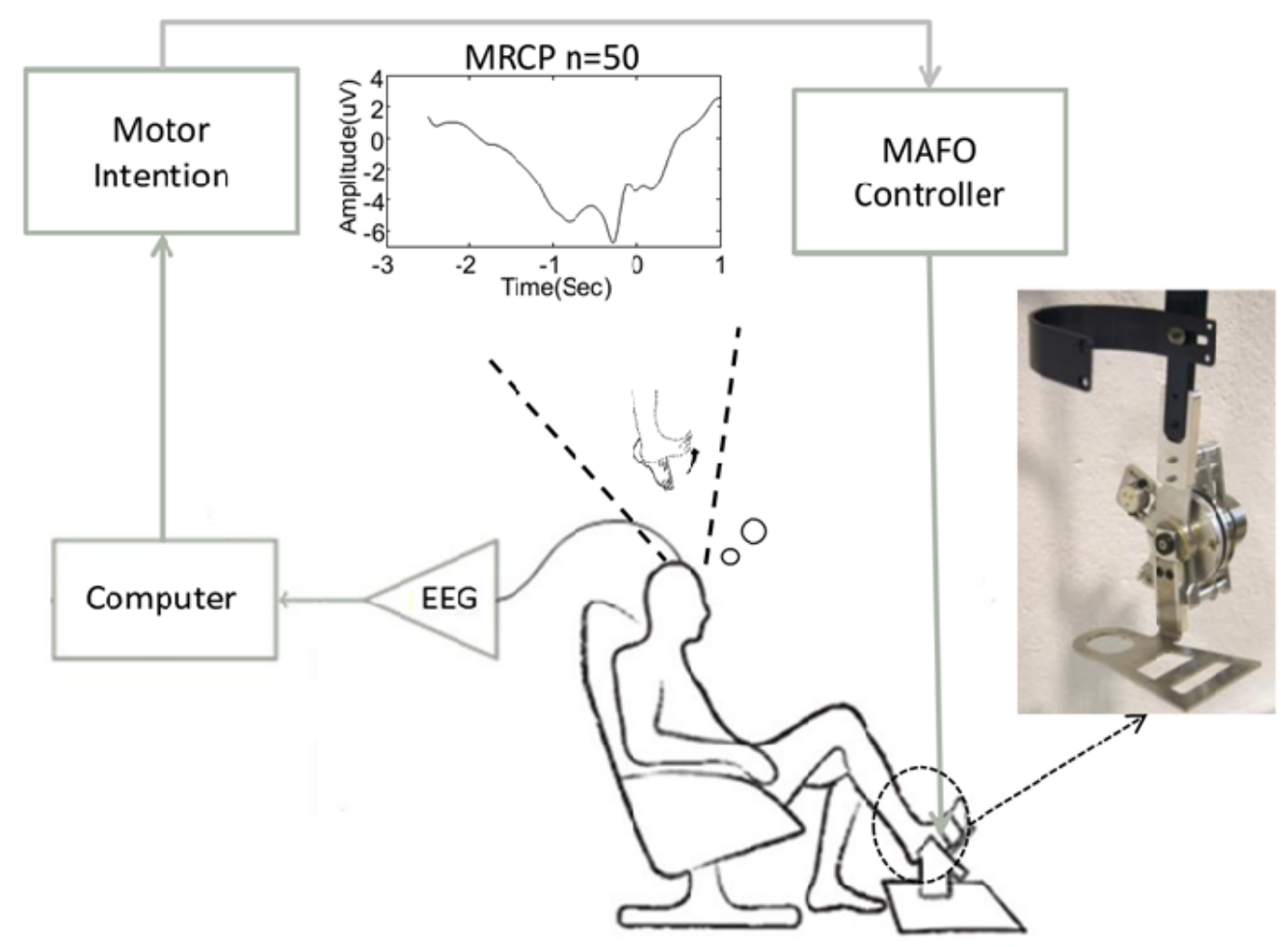

Figure 4.1: BCI-MAFO

conditions or arbitrary joint movements.

\subsubsection{BCI-MAFO intervention}

The intervention consisted of two parts (a continuous recording): a training run and a testing run. In the training run, subjects were asked to perform 30 trials of self-paced ballistic dorsiflexion (real movements) of their foot. In order to control the force output of the movement, the subjects received visual feedback of the force produced. First the subjects were asked to perform the 
MVC for $10 \mathrm{~s}$, and then they were instructed to control their force between $40 \%-60 \%$ of the MVC with a subject-view GUI. In the subject-view GUI, there is a bar indicating the force level in realtime. When the force was between the requested range (i.e. $40 \%$ - $60 \%$ ), the bar maintained green, otherwise it turned to red $(>60 \%)$ or blue $(<40 \%)$. The EEG and EMG of the training run were recorded simultaneously, and processed immediately after the training run. A brain switch was used to detect MRCPs. The brain switch was trained on the EEG data from the training run, and used online in the testing run of the intervention stage, as described below.

During the BCI-MAFO testing run, the subjects were instructed to perform imaginary dorsiflexion of the foot. The acquired EEG was processed in real time using the trained brain switch (see details in Chapter 3). Each positive detection triggered the MAFO to induce a passive dorsiflexion. The angle was $15^{\circ}$ and the velocity $40^{\circ} \mathrm{s}^{-1}$, which is in accordance with those of normal walking [9]. At the same time, a red bar appeared on the subject GUI as the visual feedback of the detection. The subjects was asked to say 'false' when the MAFO moved without a motor imagery, or 'miss' when the imagery was performed but MAFO did not move. In a pilot study, it was determined that speech of these words does not influence the proper working of the detector. The testing run of the intervention concluded when a certain number (e.g. 50) of MAFO movements had been triggered. This included true and false detections. Therefore, the number of paired events (Imagery and MAFO being triggered correctly) was always less than the number of 


\section{MAFO movements.}

The counting of true and false detections during the experiment was recorded and used to calculate the TPR and FP. The DL, defined as the time from the negative peak of single-trial MRCPs to the time corresponding to the detection, was calculated offline.

\subsubsection{The effect on MRCP morphlogy}

A pilot experiment was conducted to prove the concept of BCI-MAFO, and to investigate the effect of intervention on MRCP by comparing its morphology before and after the intervention. Three healthy volunteers (male, age: 29.3 \pm 6.6 years) participated in the experiment, and the MF was used as the brain switch for MRCP detection.

\section{Experiment protocol}

In the experiment, the subjects sat in a natural position, and wore the MAFO on the left leg. The consisted of three parts. For the first part or the pre-intervention, the subjects were asked to execute a total of 30 dorsiflexion movements following on-screen instructions by a customized user interface (synchronized mode). The MRCP template was extracted immediately from these pre-intervention EEG recordings [59]. The second part was the BCI-MAFO intervention. The subjects performed self-paced imagination of dorsiflexion (asynchronized mode). The motor imagination was detected from EEG in real time using the MRCP template extracted from 
the pre-intervention data. Upon each detection, the MAFO was triggered to perform a passive dorsiflexion at $40^{\circ} s^{-1}$ for $15^{\circ}$. The intervention ceased once a total number of 60 dorsiflexions had been triggered in this mode. The last part was post-intervention, which was the same as the pre-intervention, i.e. 30 dorsiflexion executions in the synchronized mode. Various parameters of the MRCP template, including peak-to-peak, depres-

sion rate, and rebound rate, were extracted from the pre-intervention and post-intervention data. The comparison was made to investigate if there are consistent changes from the pre-intervention to the post-intervention, which would indicate possible occurrence of cortical plasticity as a result of the BCI-MAFO intervention.

\section{Offline performance}

In this study, offline data from pre-intervention was used to gauge the MRCP detection performance through a 3-fold cross-validation. TPR of three subjects was $61.3 \%, 66.7 \%$, and $76.7 \%$, respectively. While the FP was 3.1, 2.7, and 0.8 per minute, respectively. This result was in agreement with prior offline studies [59] [60] [53].

\section{Effect of the intervention on MRCP parameters}

The peak to peak of the MRCP, depression rate prior to the MRCP peak negativity, and rebound rate following the peak negativity changed consistently from the pre-intervention to the post-intervention in all three subjects. The 

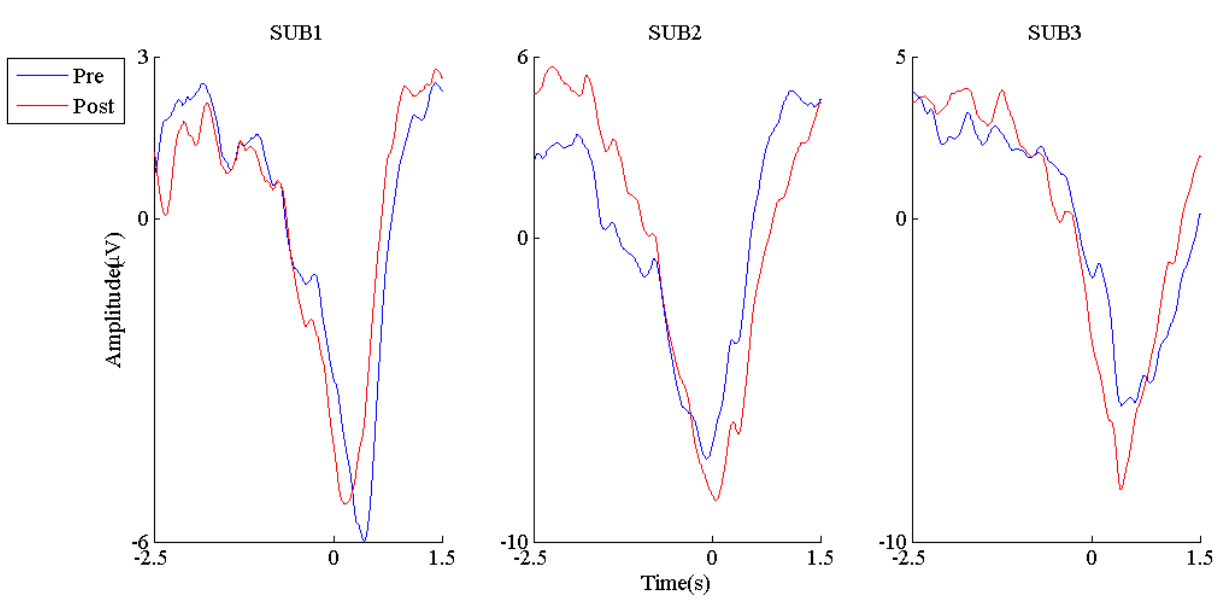

Figure 4.2: MRCP change after intervention

depression rates of the MRCP between post-intervention and pre-intervention increased by $2.4 \mathrm{~V} / \mathrm{s}, 2.3 \mathrm{~V} / \mathrm{s}$, and $2.5 \mathrm{~V} / \mathrm{s}$, respectively for the three subjects. The change of the peak-to-peak of MRCP was $3.3 \mathrm{~V}, 2.5 \mathrm{~V}$, and -2.8 V, while the rebound rates were $4.8 \mathrm{~V} / \mathrm{s},-0.9 \mathrm{~V} / \mathrm{s}$, and $4.1 \mathrm{~V} / \mathrm{s}$, respectively. As this is an ongoing study, no statistical analysis has thus so far been conducted.

\section{Summary}

In this study, we presented a BCI-MAFO ambulatory stroke rehabilitation system. The system extracts motor intentions from scalp EEG, and triggers passive muscle contractions by a wearable orthosis (MAFO). A pilot experiment demonstrated the feasibility of the system concept. Further, we showed that a short intervention of the system has the potential in inducing cortical plasticity, as indicated by the consistent changes in the MRCP parameters. 
Follow-up studies are underway to further investigate the potential of the proposed system, including fine tuning the experiment protocols and MAFO parameters.

\subsubsection{The effect on neural plasticity}

Following the pilot investigation of the effect on MRCP morphology, we conducted a full experiment with proper controls, to study the effect of BCIMAFO intervention on neural plasticity.

\section{Main Experiment}

Ten healthy subjects (9 male and 1 female, age: $28.1 \pm 4.2$ yrs) participated in this study. All but one subject were naïve to MRCP-based BCI systems. Nine of them participated in experiments consisting in three intervention conditions: BCI-MAFO, BCI-alone, and MAFO-alone (one male subject did not complete the latter two conditions). In addition, four of the subjects took part in the stretch-reflex and active MEP experiments (see below). Subjects were seated in a custom-made chair, and placed their right foot in an instrumented pedal, from which the torque at the ankle was measured by a torque transducer (Aalborg University, Aalborg, Denmark). Initially the optimal spot for TMS was identified using approximatley $50 \%$ of the stimulator output. This site was defined as that spot on the motor cortex where the motor evoked potential (MEP) remained consistent in amplitude within three consecutive stimuli and where the largest MEPs were attained 


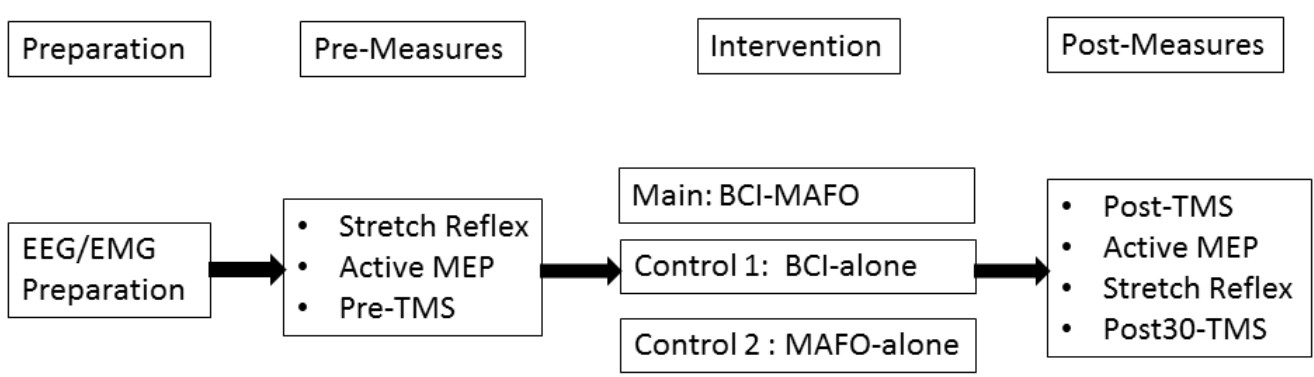

Figure 4.3: BCI-MAFO experiment protocol

within the TA. This spot was marked with a felt pen to ensure that pre, post and 30 min post stimuli were applied to the same area. Next, the resting threshold (RTh) was measured, as that intensity where 5 out of 10 consecutive stimuli provided MEPs above $50 \mu \mathrm{V}$. Subsequently, stimuli were delivered at $90,100,110,120$, and $130 \%$ of RTh, in random order. Ten stimuli were delivered at each level of intensity, with an interval between stimuli of 5-7 s. These were repeated immediately and $30 \mathrm{~min}$ following the intervention outlined below.

During the pre-measures, active MEPs and stretch reflexes and pre-TMS were induced as outlined above and in Fig 1. The post-measures stage would be performed after the intervention stage (in all three conditions). In addition to active MEPs and stretch reflexes, two TMS-measurements were performed: one immediately after the intervention stage (post-TMS), and another one $30 \mathrm{~min}$ after the intervention stage (post30-TMS), to determine the duration of the induced effects. 


\section{Control interventions}

Two control interventions were also performed, in which the BCI-MAFO intervention was replaced by either BCI-alone, or MAFO-alone. This was to ensure that any changes in the excitability of the cortical projections to the TA were indeed due to the main intervention. During the BCI-alone condition, the subjects performed the same tasks as those in the BCI-MAFO condition. The only difference was that no passive movements were induced. During the MAFO-alone condition, the subject did not perform the motor imagery task, and the MAFO moved randomly, with similar random intervals as the other two experimental conditions. For both control interventions, TMS measures at rest were performed prior to, immediately following and 30 min after the cessation of the intervention.

\section{Stretch reflex and active MEP measurements}

To investigate if any plasticity occurred at the spinal level following the intervention, stretch reflex and active MEP measurements were included as part of the pre- and post-measures for 4 subjects. For the stretch reflex measurements, the right leg of the subjects was affixed to a servo-controlled hydraulic actuator (MTS-systems Corporation, 215.35), such that the anatomical ankle axis of rotation was closely aligned with the fulcrum of the actuator. The foot segment of the right leg of the subject was firmly strapped to a custom made plate that extended from the actuator, thus producing a tight

interface between the arm of the motor and the foot of the subject, ensuring 
that the movement of the actuator was transmitted solely to the ankle joint. The angular position of the actuator was monitored by an angular displacement transducer (Transtek, DC ADT series 600). The subjects were asked to maintain a $5 \%$ MVC in their right TA while the perturbations were applied. The EMG level was displayed on a computer screen placed in front of the subject. The instructions to the subjects at all times were to maintain the $5 \%$ MVC EMG level without interfering with the imposed plantar-flexion perturbation.

Thirty stretches were randomly applied at intervals ranging from 5 to $7 \mathrm{~s}$ (velocity of $200^{\circ} \mathrm{s}^{-1}$ to $250^{\circ} \mathrm{s}^{-1}$; amplitude: $6^{\circ}$; hold-time $\left.460 \mathrm{~ms}\right)$. The angular velocity and the amplitude of the imposed perturbations were adjusted for each subject so that the amplitude of the response peaks observed in the TA EMG trace were approximately the same and also similar to the amplitude of the active TA MEP prior to the intervention. The latencies of the first response peak, termed short latency response (SLR) in the literature, were extracted from the data both prior to and immediately following the intervention. The root mean square (RMS) value of a window extending $10 \mathrm{~ms}$ on either side of SLR (thus $20 \mathrm{~ms}$ for each window in total) was calculated and used as an indication of the size of each of this purely spinal component of the TA stretch reflex. Post measures were expressed as a percentage of the pre values.

Active MEPs were recorded prior to and immediately following the intervention while the subjects maintained a TA contraction of $5 \%$ MVC. For the 
pre-measures, care was taken to ensure that the amplitude of the rectified MEP was approximately matched to the amplitude of the SLR component of the stretch reflex. This was to ensure that the motor units targeted by an imposed stimulus (mechanical or electrical) were from a similar pool. The magnitude of the MEPs measured was determined from the averaged RMS value of the area under the rectified MEP.

\section{Statistical analysis}

Three-way repeated measure ANOVA was used. The three main factors were intervention condition (BCI-MAFO, BCI-alone and MAFO-alone), TMS in-

tensity (5 TMS stimulation levels), and TMS measurement timing (pre, post and post30). Full model ANOVA with all interaction terms was performed first and, when significant interactions were detected, focused ANOVA tests were performed.

\section{BCI performance}

The BCI performance measures of the BCI-MAFO intervention, i.e. TPR, FP and DL, are presented in Table 1. The duration of the testing run was $13.9 \pm 3.8 \mathrm{~min}$. TPR, FP and DL were $73.0 \pm 10.3 \%, 34.2 \pm 9.2 \%$, and 14 $\pm 236 \mathrm{~ms}$, respectively. Thus the movement intention was detected around the peak negativity of the MRCP, which coincides with the onset of the movement imagination. For five subjects, the detections were 213-365 ms before the peak negative, while other three were less than 200 ms afterwards. 


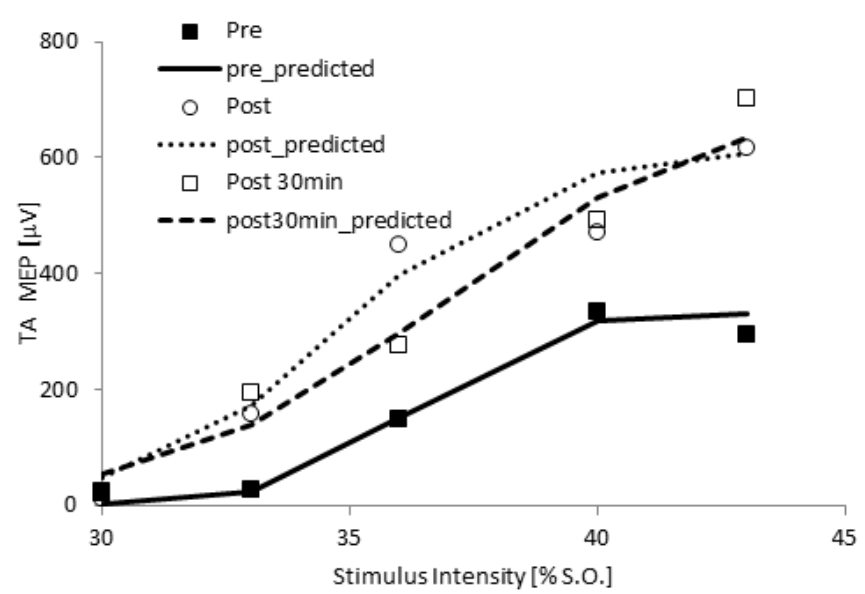

Figure 4.4: MEP change agter intervention: single subject

These intervals were within the range of effective intervention according to the study by Mrachacz-Kersting et al. [53].

\section{Changes in the excitability of the cortical projections to the TA}

The MEP of a typical subject before, immediately after, and 30 min after the BCI-MAFO intervention is illustrated in Figure 4.4. Each point represents the median value of the MEP size (peak-to-peak value) of the 10 stimuli at each of the five stimulation intensities. The markers indicate the timing of TMS measurement, i.e. pre, post or post30. Following the BCI-MAFO intervention, there were clear increases in the MEP amplitude at all stimuli intensities from pre-measure to the two post-measures. This indicates that this short-time intervention induced evident plasticity, which out-lasted the intervention. 


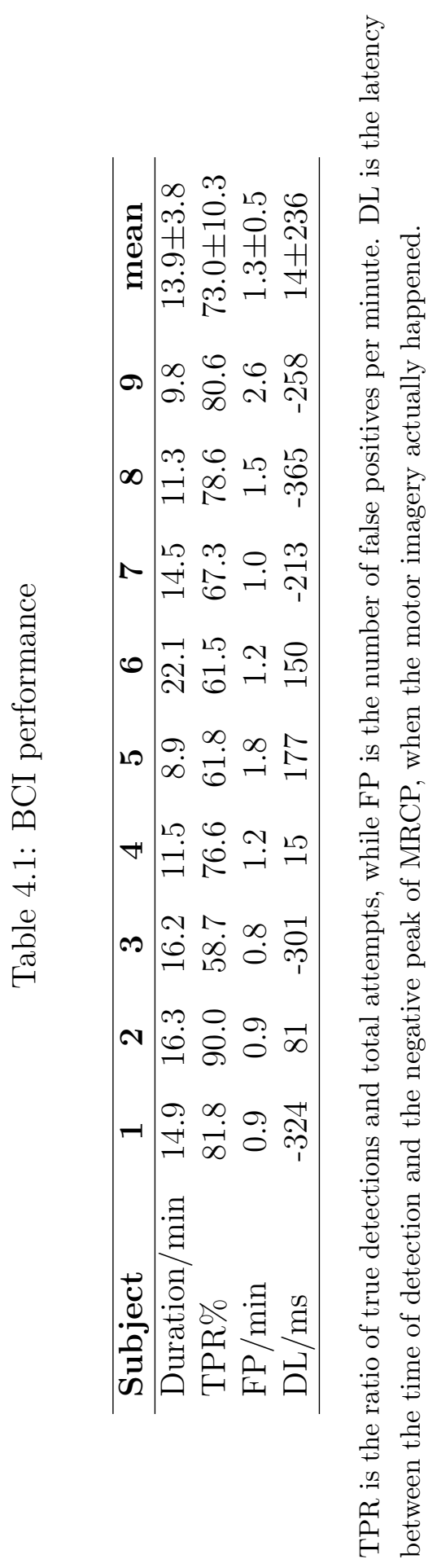




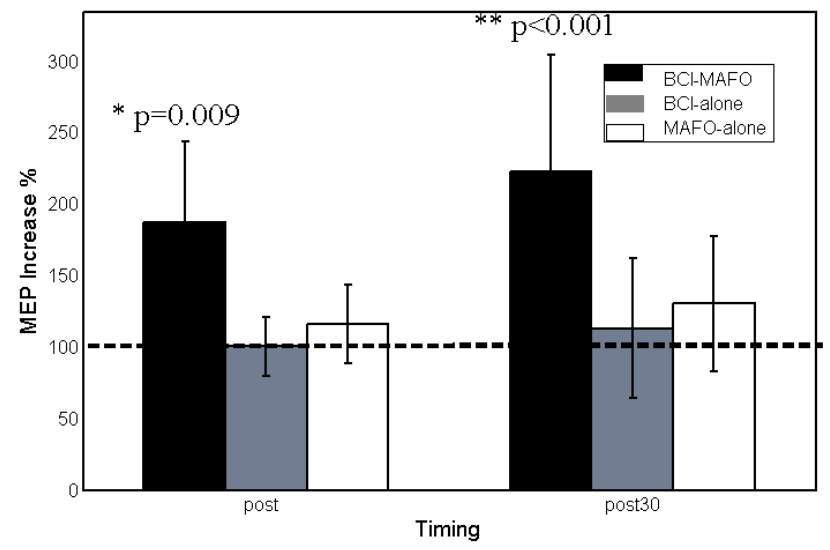

Figure 4.5: Comparison with controls

The full-model ANOVA revealed a significant interaction between intervention condition and TMS measurement timing $(p=0.026)$, while no other interaction was detected $(p=0.51$ and $p=0.56$ for the other two-factor interactions, and $p=0.87$ for the three-factor interaction). Subsequently, focused ANOVA tests were performed on each of the three intervention conditions. Whenever significance of a main factor was detected, post-hoc multiple comparison (Tukey simultaneous test) was performed. In all three focused ANOVA, no interaction between TMS intensity and TMS measurement timing was found ( $p=0.40,0.99$ and 0.59 , respectively), so reduced ANOVA only considering the main effects were performed. For BCI-MAFO, TMS measurement timing was found to be a significant factor $(p<0.001)$. Post-hoc comparison found that post and post-30 were significantly larger than pre $(p=0.009$ and $p<0.001$, respectively), and there was no statistical difference between post 
A Ankle Angle

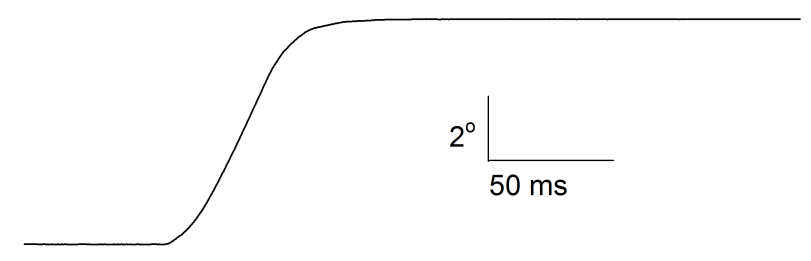

B TA stretch reflex SLR MLR

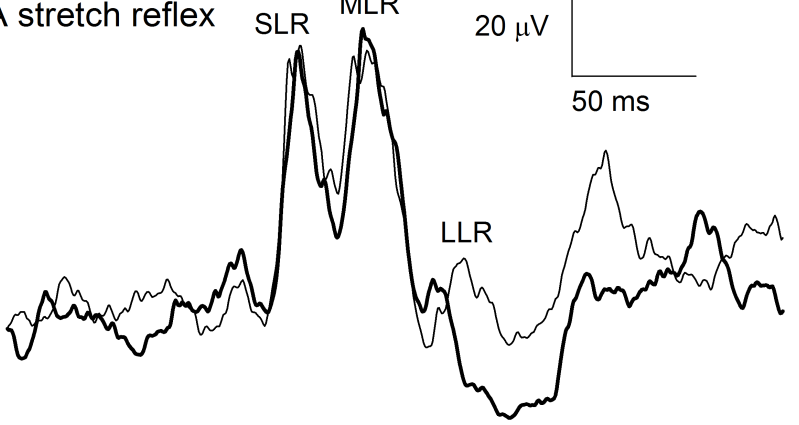

C TA active MEP

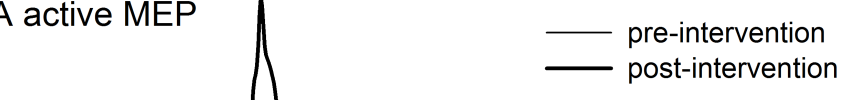

Figure 4.6: Stretch reflex and active MEP 
and post-30 $(p=0.38)$. Conversely, for both BCI-alone and MAFO-alone, TMS measurement timing was not a significant factor $(p=0.38$ and 0.15$)$. In four subjects, the changes in the TA stretch reflex parameters were compared to the changes of the TA MEP elicited during a similar level of TA contraction (i.e. $5 \% \mathrm{MVC}$ ) (see also Figure 4.6). The RMS value for the background level of activation was $36.0 \pm 11.0 \mu \mathrm{V}$ for the MEP trials and $36.3 \pm 9.8 \mu \mathrm{V}$ for the stretch reflex trials prior to the intervention. Post intervention, these values were $38.2 \pm 9.0 \mu \mathrm{V}$ and $35.0 \pm 9.0 \mu \mathrm{V}$. The $\mathrm{TA}$ active MEP increased across all subjects by $64 \pm 32 \%$, although as only four subjects were examined this did not reach statistical significance $(p=$ 0.22). The SLR component did not change, being on average $100 \pm 15 \%$ of the pre-intervention value following the intervention.

\subsubsection{Comparison with BCI-ES}

As a further investigation on the influence of different types of sensory feedback on the efficiency of plasticity induction, three subjects (1 female, age: $26.7 \pm 1.2 \mathrm{yrs}$, who have already participated in the BCI-MAFO experiment, were recruited for a comparison study.

\section{Experiment}

In this study, an intervention by BCI driven electrical stimulation (BCI-ES), similar to what was proposed in [60], was introduced. The BCI trigger used here was LPP-LDA, the same as that in BCI-MAFO intervention. A sub- 
threshold ES was delivered through an isolated stimulator (TremUNA, Una Sistemi, Serbia) to the nerve for TA muscle. The subject can only feel the sensory input by ES, but without any muscle contraction.

As done in the BCI-MAFO experiment, a pre-, post- and post30-TMS measurement was performed to qualify the cortical output, and compared with the results from the BCI-MAFO intervantion.

\section{Results}

As shown in Figure 4.7, the BCI-ES intervention yielded an increased MEP of $54 \pm 75 \%$ in the post-measurement, and the increase expanded to $131 \pm$ $158 \%$ after 30 minutes. For BCI-MAFO intervention on the same subjects, the MEP increased $95 \pm 104 \%$ and $67 \pm 69 \%$ in the post- and post30TMS, respectively. No statistical analysis was performed due to the limited number of subjects.

\subsubsection{Discussion}

In this study, we demonstrated that the proposed BCI-MAFO system induced plasticity following a very short intervention $(\sim 14 \mathrm{~min})$, during which self-paced motor imagery was detected online and used to trigger the MAFO to elicit passive dorsiflexion movements. The MEPs increased significantly

following and 30 min after the BCI-MAFO intervention, while there was no change in the M1 components of the TA stretch reflex. This indicates that the site of the plastic change was likely supra-spinal. 


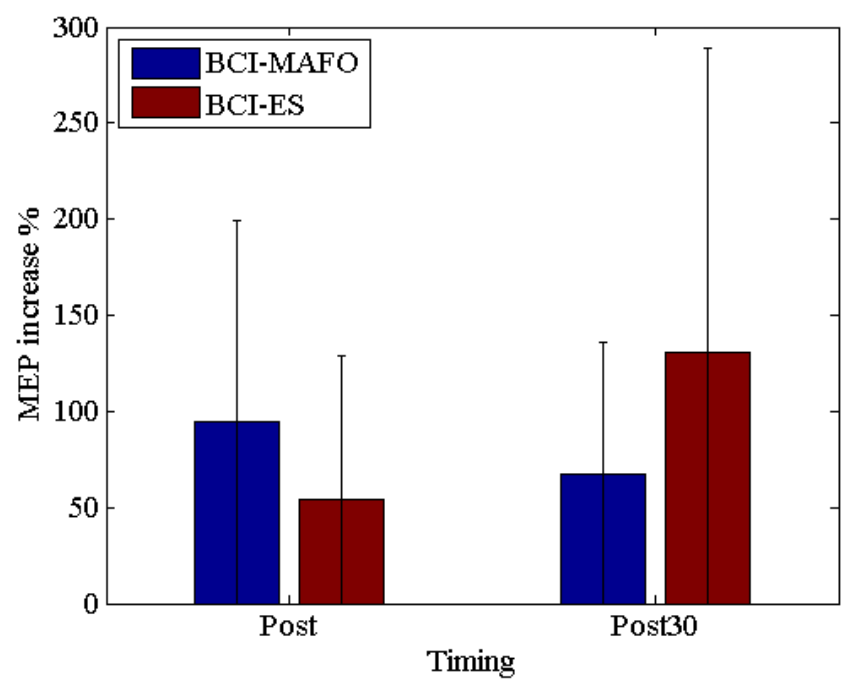

Figure 4.7: Comparison with BCI-ES

\section{BCI for rehabilitation}

In the past decades, the BCI studies for motor function rehabilitation have focused on SMR, since its desynchronization and synchronization (ERD/ERS) were found to be modulated by motor imagery [66]. In these studies, SMRbased BCI was used to provide visual feedback or to trigger external devices, such as ES or orthoses, aiming at the rehabilitation of motor functions [73][8][3]. Cortical plasticity did occur through a number of interventions over the course of a number of weeks [73]. In Ramos-Murguialday et al. [73], the intervention was conducted over a four week period with daily training. In Pichiorri et al. [69], the intervention consisted of six to eight 40-min long training sessions over a four-week period. Plasticity was not observed in all 
subjects.

In the current study, we showed that only one $\sim 14$-min intervention was necessary for the proposed BCI-MAFO system to induce significant cortical plasticity, quantified by increases in the TMS-induced MEP. The main difference between the approach used in the current study and the SMR-based BCI approaches relates to the timing of motor intention detection. Contrary to SMR-based BCI systems, the motor intention can be detected with high precision using the proposed MRCP approach. Therefore, the artificial sensory feedback reaches the cortex at almost the same time when the subject imagines the movement, This timing had been demonstrated to be crucial in inducing plasticity [53]. If the afferent volley arrived 400-500 ms too late or too early with respect to motor intention, the Hebbian association would not be satisfied [53]. Consequently the LTP-like effects, the primary association in plasticity, memory retention and learning process [31], would not be induced. Therefore, the latency between motor imagery and associated feedback is an essential factor of an effective BCI system in rehabilitation. In addition, all but one subject of the current study were BCI or EEG-naïve subjects in the sense that they never participated in any experiments involving BCI and EEG measurements. They were able to produce an MRCP in the first session naturally. MRCP is indeed generated in a variety of movements [19], and thus it is applicable to naïve subjects even without training [93]. This property is especially desirable for ambulatory applications in household environment. 


\section{Previous studies on MRCP-based systems}

There have been two paradigms proposed for MRCP-based BCI systems for rehabilitation. In the first, which was cue-based or synchronized, the subjects were asked to perform motor execution or imagery according to a cue within the subject-view GUI [53]. The second, which was a self-paced BCI, was applied by Niazi et al. [59]. Compared with cue-based paradigms, self-paced $\mathrm{BCI}$ is more natural because the user, rather than the machine, controls the timing of operation. This kind of totally patient-driven system with full voluntary involvement is more suitable for ambulatory applications. This is the reason why this modality was chosen also in the current study.

In this study, we proposed a self-paced BCI-MAFO system, and demonstrate for the first time the high efficiency in inducing plasticity when an active orthosis is triggered by brain waves within a precise temporal accuracy. The MEPs increased on average by $87.2 \%$ after the intervention with less than 50 imagery attempts in $\sim 14 \mathrm{~min}$, while in the study by Niazi et al. [59]

the MEPs increase was smaller $(53 \%)$, even if more attempts $(\sim 75)$ and longer time $(\sim 27 \mathrm{~min})$ were dedicated to the intervention. The improved performance of the current system is likely due to the improvement in the key components, as outlined in the following.

The first key improvement was the detection accuracy. The LPP based classifier used in this study yielded a higher performance, with $73.0 \pm 10.3 \%$ of TPR and $1.3 \pm 0.5 / \mathrm{min}$ FP in BCI-MAFO condition, comparing with pre- 
vious studies on MRCP detection [59][60][58]. Based on the previous result that the plasticity increase was linearly associated with BCI performance [60], we could speculate that the high performance of the new detection algorithm played an important role in the efficiency of plasticity induction shown in the current study. Importantly, the improved detection accuracy was obtained without increasing the detection latency, which still allowed the triggering the MAFO within a few hundred ms, from the instant at which the movement would be executed.

The natural sensory feedback provided by MAFO was another key component of the current system. The single ES applied in our previous studies was set to motor threshold which means that mainly low-threshold afferents were recruited, as the threshold for the other afferents, such as cutaneous afferents, is higher [80]. In addition, we applied it to one nerve that innervates the target muscle TA. By using MAFO, we stretched the target muscle and unloaded the antagonists, both of which may result in alterations in afferent feedback. We also recruited non-muscle afferents (mainly cutaneous afferents) and these may also be involved in the larger effects induced [61]. An important advantage in using joint movements to provide the afferent feedback lies in the fact that cutaneous and other high-threshold afferents have been implicated to play a large role in contributing to muscle activation during human walking [80][61], thus the type of feedback that the MAFO can supply is much more realistic. 


\section{Conclusion}

A BCI-MAFO system aimed at stroke rehabilitation was presented in this study, and its ability in inducing cortical plasticity with very short intervention time was shown in nine healthy subjects. The combination of a high performance BCI algorithm (i.e. LPP-LDA) and natural sensory feedback produced by MAFO resulted in a very effective way in increasing cortical excitability. Based on these results, the BCI-MAFO system could potentially be an ambulatory rehabilitation tool for stroke patients.

\subsection{Brain switch triggered selective electro- tactile menu for communication}

\subsubsection{Motivation}

Besides rehabilitation purpose, BCI has been developed as a assistive tool for replacing impaired functions of severe disabled people [18]. For example, a BCI system detects the users intention from the brain signal, e.g. EEG, and decodes it into computer command, either for communication or motor control [78]. For these purpose, various EEG modalities have been investi-

gated, including evoked potentials which were elicited externally by visual, auditory, or tactile stimuli, or event-related potentials produced by initiative motor or mental tasks.

Among those communication systems developed based on te above EEG sig- 
nals, gaze-dependant systems with visual or auditory P300 is not applicable to patients with visual or auditory impairment [75]. SSEP based systems are limited by the number of class, since the classification performance decease dramatically with the increase number of class. Multiple-MI systems have the same drawback as SSEP, and usually need intensive subject-training to form a distinguish EEG pattern among different motor tasks. Tactile P300 seems a promising approach to extensive users, but due to the similar eventrelated potentials between target and non-target stimuli, it usually needs to average several trials for higher accuracy.

In a series of recent studies, our group has been developing a fast, accurate and robust brain switch using MRCP [40]. The stable property of MRCP was demonstrated in healthy subject [93], as well as stroke [58] and spinal cord injured patients [97]. We further developed algorithms for robust detection of MRCP, with a true positive of 80\% and a latency of 300 ms [93]. This brain switch was already applied to control of external devices such as electrical stimulator and robotic [60][96], and its high efficiency in stroke rehabilitation was approved [54].

In this study, we attempt to extend the application of this versatile brain switch to communication purpose, aiming at developing a novel electrotactile BCI menu. With electrical tactile on the neck, it would be applicable to vast target user, such as patients with visual or auditory impairment, or high-level paraplegic patients without sensory function below neck. More importantly, the BCI menu is triggered by such a robust brain switch, which 
detects the motor intention fast and accurately by single-trial analysis of MRCP. Thus it would be initiative, and with barely limitation on number of class.

\subsubsection{BCI menu setup}

\section{Electrical stimulator}

The ES was delivered by an 8-channel current-controlled electrical stimulator (TremUNA, Una Sistemi, Serbia). This is a fully programmable stimulator designed for functional electrical stimulation (maximum stimulation intensity: $5 \mathrm{~mA}$ ). The unit is batter powered and fully isolated from the mains. Standard self-adhesive, disposable stimulation electrodes were utilized (PALS Platinum and PALS Valuetrode, Axelgaard Manufacturing, US).

\section{Stimulation location}

Two locations, i.e. the fingertips and the neck, were investigated in a pilot study on one subject. The main aim of this pilot is to investigate the ES artifacts on EEG.

For the fingertips, the stimulation electrodes were cut into small pieces, leaving only the central positive pole, to fit the small available area. Four of such custom-made electrodes were placed on index, middle, ring and small fingertip, respectively. A fifth electrode with only negative pole was placed on the wrist as the common reference of all those four stimulation electrodes. For the neck, since there were enough space for the electrode, four original 
electrodes, without any further process, were located in front, right, back and left area of the neck.

The experiment consisted of two parts: 1) individual setting of ES threshold, which can be clear distinguished by the subject without any discomfort; 2) self-paced movement of dorsiflexions with ES on ad off, to test the effect of ES on the EEG signal.

The results were shown in Figure 4.9. For stimulation on the neck, there was clear stimulation artifact on raw EEG signal, while nothing visible for the locations of fingertips. However, after the band-pass filter and spatial filtering, the MRCPs with stimulation on these two locations looks very similar to each other.

The main advantages of locations on fingertip is that it produced less artifact, which may due to its larger distance to brain, as well as its lower stimulation threshold, when compared to that of the locations on the neck. On the contrary, the stimulation on the neck produced strong artifact on raw EEG, but were almost removed by these filters, resulting an comparable MRCP with that from ES located on fingertips. More importantly, the locations on the neck has broader applications, especially for some paraplegic patients those who preserve sensory sensation above neck level. Given this high priority, we consider the ES locations on the neck as a better solution, and used it the BCI menu in the following study.

\section{ES menu}




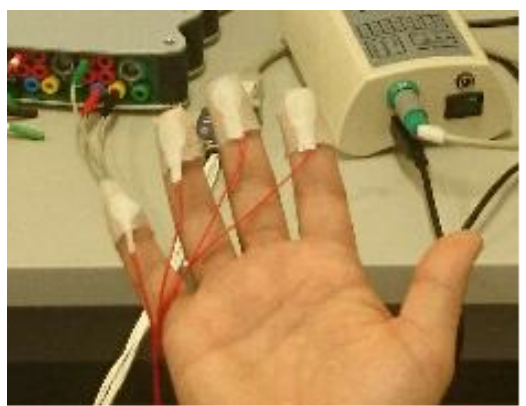

(a) Fingertips

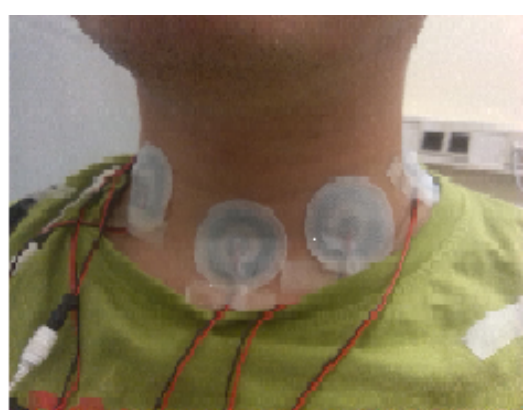

(b) Neck

Figure 4.8: ES locations
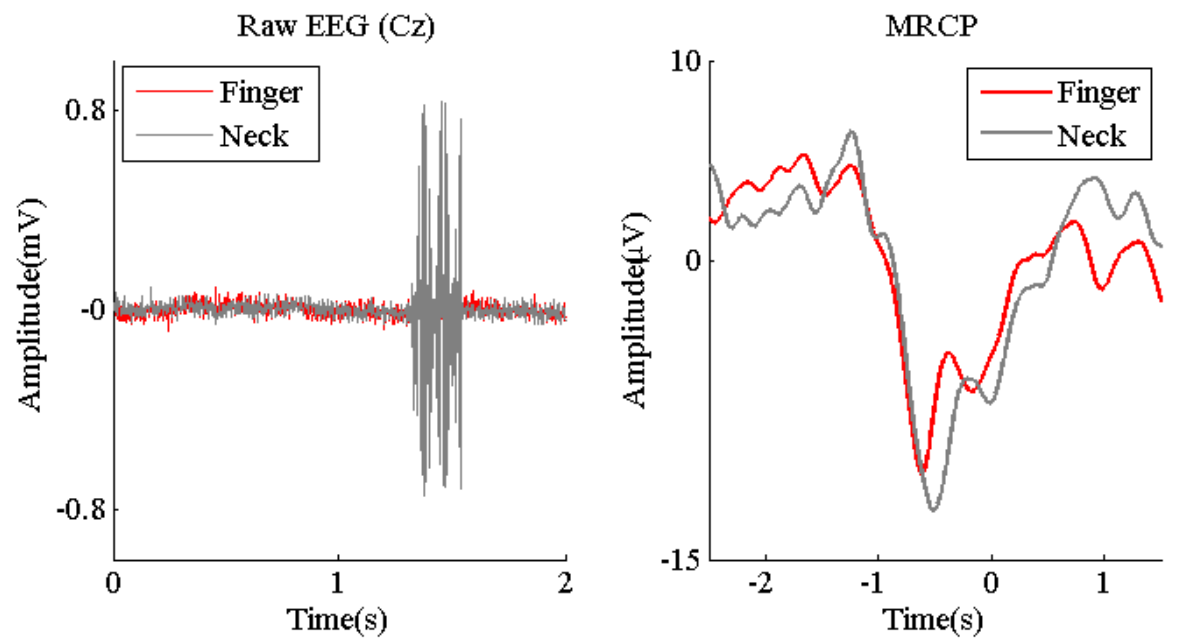

Figure 4.9: ES artifact 
1) Four-class menu

As shown in Figure 1, 4 stimulation electrodes (No. 1-4) were located clockwise around the neck as the following sequence: front, right, back and right. The stimulation lasted $0.2 \mathrm{~s}$ at each location, and the interval between each two stimuli was $1.8 \mathrm{~s}$, making up a 8 s stimulation with $2 \mathrm{~s}$ per menu.

2) Eight-class menu

An 8-class menu was presented as an extended version. Similar to the 4-class menu, eight stimulation electrodes were placed around the neck clockwise with equal distance. The stimulation interval was shorten to $1.5 \mathrm{~s}$, including a stimulation interval of $0.2 \mathrm{~s}$ (same as 4-class menu) and a rest interval of 1.3s between each two stimuli. Therefore, each cycle of the 8-class menu lasted $12 \mathrm{~s}$.

For both 4-class and 8-class menu, the stimulation intensity was set individually at each stimulation location, to make sure the subject could clearly identify the stimulation at each location without any pain or uncomfortable sensation.

\section{BCI trigger}

The BCI is triggered by the short-latency brain switch, which was presented in the previous chapter and thus not repeated here. 


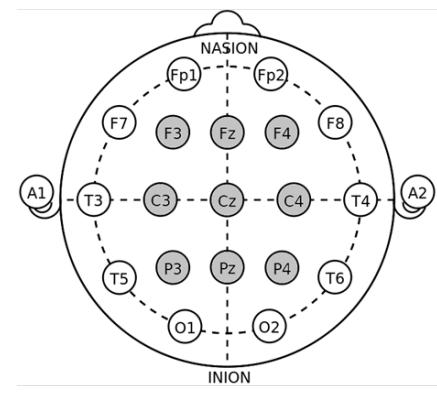

(a) EEG channels

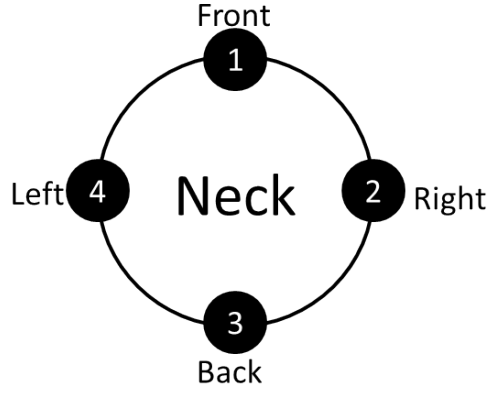

(b) ES locations

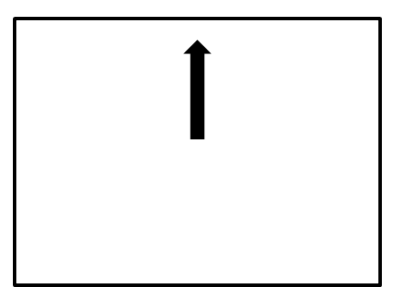

(c) User view

Figure 4.10: BCI menu setup

\subsubsection{Experimental Procedure}

\section{Main protocol with 4-class menu}

The main experiment consisted of a training session and two test sessions. In the training session, the subjects sit in a comfortable chair, with the right foot fixed on a self-made pedal. They were instructed to perform self-paced dorsiflexions of their right foot. The intervals between every two of the movements were control by the subjects, who may have a short rest whenever they felt tired. The MRCPs produced by each movement were presented to the experimenter simultaneously, and those abnormal trials, which were obviously corrupted by unintended movements (e.g. eye blinking), were marked and removed from following analysis. After removal, around 30 trials were recorded and used to train the LPP-LDA classifier.

The first testing session was a movement execution (ME) session, in which the subjects performed real movements of dorsiflexion, the same as they did 
in the training session, to trigger the BCI. It includes four phases: passive mode, BCI phase, passive mode with ES, and BCI-menu phase.

1) Passive phases

In the passive mode (with or without ES), the subjects were asked to be relax for one minute. Unintended movements such as eye blinking and swallowing were allowed in this passive mode, and the number of passive false positives (pFP) was counted as a robust index of the algorithm. 2) BCI phase

The BCI phase, which was the same as what have been done in [93], is to verify the detection performance and set the benchmark for BCI-menu. In this phase, the subjects performed 30 trials of dorsiflexions, and the number of true detections and false detections were counted. 3) BCI-menu phase The last phase, i.e. BCI-menu, is also the main part of this experiment. In order to provide a closed-loop feedback, a subject-view was shown in the monitor. A black arrow appeared in the screen, pointing to front, right, back or left, indicating the number of channel that the subject should choose. For example, the subject would be required to perform dorsiflexion after the front stimulation if the black arrow was in the front direction. When the subject performed properly and his/her motor intention was detected within the current menu (i.e. the period after the current stimulation onset, and before the next stimulation), the arrow would turn to green, indicating a successful task. Otherwise a red arrow would appear in another direction, showing a false task there, which could be induced either by ill-timed movement of the subject or false detection of the algorithm. As all the subjects 
were the first time to the BCI-menu system, an adaptation process ( $\sim 5 \mathrm{~min}$ ) was performed, in which the subjects were instruct to get familiar with the system. In the BCI-menu phase, 2 runs were performed after adaptation. Each run consists of 40 randomized tasks (10 repeats at each ES location). The second testing session was almost identical to the first one, but the BCI was triggered by MI of dorsiflexions without any real movements. It also includes four phases as above, but there were some differences due to some specific nature of motor imagery. In the BCI phase, the subjects were required to say 'YES' after imagination, which was used for counting the number of attempted motor imageries. Yet this requirement was not executed in the BCI-menu, avoiding multiple tasks for the subjects.

The second testing session is even more relevant to real applications on patients, who could barely perform real movement and thus the motor imagery is the only output. However, as there is no direct reference for self-paced imagination, the objective measurement of performance is difficult. As such, the first testing session is also essential for system verification, where the timing of real movements were recorded with EMG.

\section{The extended experiment with 8-class menu}

The extended experiment was identical to the main protocol, but with the increased menu number (8-class) and a reduced between-class interval (1.5s). It also includes a training session and two testing session, but only the BCImenu phase was performed in the testing sessions. In addition, since the 


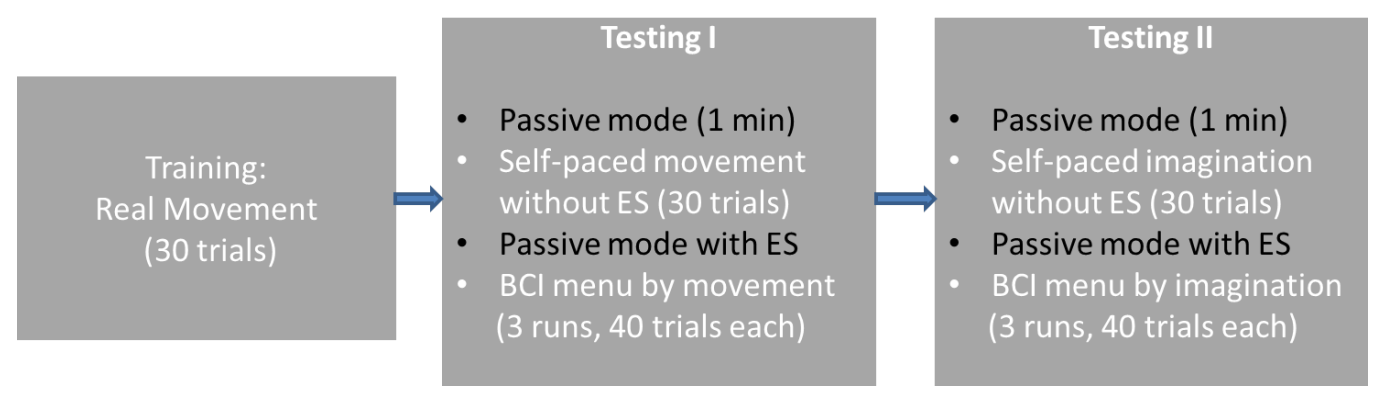

Figure 4.11: BCI menu experiment

two subjects have already taken part in the main protocol, the adaptation process in the BCI-menu phase was not performed here. Besides, only one run of BCI-menu (both execution and imagination) was performed as a proof of concept.

\subsubsection{Evaluation criteria}

\section{Detection performance}

The TPR and FP were calculated for measuring the performance in the BCI phase. TPR was the percentage of the number of detected movements or imaginations with respect to the total number of attempts. In the BCI and BCI-menu phase, aFP (active false positive) was the number of false detections per minute. Similarly, pFP is the false positives in the passive mode. For BCI-menu phase, FP was calculated the same as above, while the TPR was estimated by the ratio of the number of corrected tasks and total tasks. In addition, the latency was calculated as the interval between the onset of ES and when the detection happened, including the duration of ES (0.2s), 
the response time from the subjects, and the detection latency from the algorithm.

\section{Information transfer rate}

As usual in BCI based communication system, ITR was calculated for further evaluate the performance of BCI menu. It is defined as follow:

$$
B=\log _{2} N+P * \log _{2} P+(1-P) \log _{2}((1-P) /(N-1))
$$

Where $\mathrm{B}$ is the ITR in bites per symbol, $\mathrm{N}$ is the number of class, and $\mathrm{P}$ is the accuracy. More frequently, given the time needed for transferring a symbol (T), ITR is calculated in the form of bits per minute:

$$
I T R=B /(60 / T)
$$

\section{Statistical analysis}

A Mann-Whitney U-test was performed on the performance, i.e. TPR and aFP in the BCI phase. The variable is the motor task: movement or imagination. The same statistical analysis was performed on the comparison of pFP with or without ES.

A two-way ANOVA was utilized for comparison of BCI- menu performance (TPR, FP and latency). Two variables are motor task (movement or imagination), and number of runs (2 levels). Once the interaction was found, a focus ANOVA on single variable would be performed. 


\subsubsection{Results}

\section{MRCP morphology}

As shown in Figure 4.12 (a), the strong artifact from ES were almost fully removed from EEG after the pre-processing steps, i.e. band-pass and spatial filters. The MRCPs with and without ES were compared in Figure 4.12. It is clear that there was no obvious effect from ES, resulting the identical MRCP morphology.

\section{BCI performance}

The detection performance in the BCI phase was shown in Table I. For most subjects, the TPR was above $70 \%$, and the aFP was below 2 per minute. The pFP was a bit higher, around 3 per minute, though. No significance was found for TPR between ME and MI and $\mathrm{aFP}$ ( $\mathrm{p}=0.30$ and 0.80 , respectively), nor between for $\mathrm{pFP}$ with or without $\mathrm{ES}(\mathrm{p}=0.97)$. Thus we polled the results together, and got the average TPR, aFP and pFP of $74 \pm 10 \%, 2.0 \pm 1.0$ $\min ^{-1}$ and $2.9 \pm 1.1 \mathrm{~min}^{-1}$, respectively.

\section{BCI-menu performance}

1) Four-class BCI-menu performance

The performance of the 4-class BCI menu, including TPR, FP, latency and ITR, was shown in Fig.4. The TPR was $\sim 80 \%$ and $\sim 70 \%$ for ME and MI, 

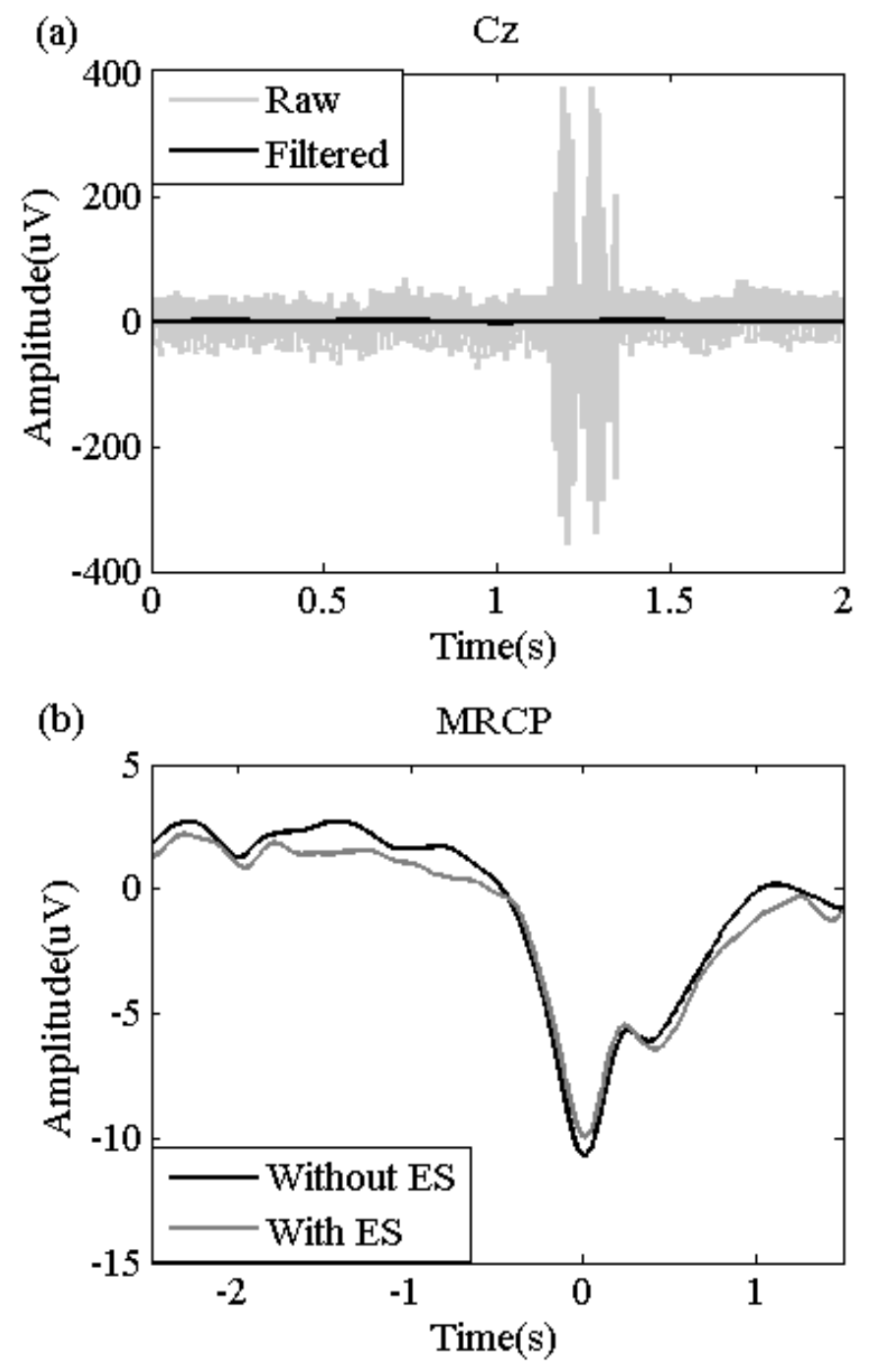

Figure 4.12: MRCP morphology with/without ES 
Table 4.2: Detection performance

\begin{tabular}{c|cccccc}
\hline & \multicolumn{2}{|c}{ TPR \% } & aFP & min & \multicolumn{2}{c}{ pFP $/$ min } \\
\cline { 2 - 7 } SUB & ME & MI & ME & MI & NoES & ES \\
\hline $\mathbf{1}$ & 85 & 70 & 0.4 & 0.8 & 0 & 2 \\
$\mathbf{2}$ & 80 & 77 & 1.6 & 1.2 & 3 & 3 \\
$\mathbf{3}$ & 87 & 70 & 2.0 & 1.6 & 4 & 3 \\
$\mathbf{4}$ & 58 & 71 & 4.4 & 2.8 & 2 & 2 \\
$\mathbf{5}$ & 90 & 67 & 2.8 & 2.4 & 4 & 4 \\
$\mathbf{6}$ & 56 & 77 & 1.5 & 3.3 & 3 & 4 \\
$\mathbf{7}$ & 74 & 71 & 1.9 & 1.5 & 4 & 3 \\
\hline Mean & $\mathbf{7 4 \pm \mathbf { 1 0 }}$ & $\mathbf{2 . 0} \pm \mathbf{1 . 0}$ & $\mathbf{2 . 9} \pm \mathbf{1 . 1}$ \\
\hline
\end{tabular}

respectively, while $\mathrm{FP}$ was $\sim 2$ per minute. These results were similar to the BCI performance. The two-way ANOVA found no interaction $(p=0.446$, 0.119, 0.904, 0.397, respectively) between motor task and run, nor no significance ( $p=0.729,0.626,0.487,0.403$, respectively) between two runs, for each performance criteria (i.e. TPR, FP, latency and ITR). Therefore, in the following, we polled the results from two runs together.

TPR with ME was found significantly higher than that with MI (78 $\pm 8 \%$ vs $69 \pm 11 \%, p=0.017)$. This significance also applies to latency $(1.00 \pm 0.15 \mathrm{~s}$ vs $1.11 \pm 0.13 \mathrm{~s}, p=0.010)$, and ITR $(7.3 \pm 2.2 \mathrm{bits} / \mathrm{min}$ vs $4.9 \pm 2.6 \mathrm{bits} / \mathrm{min}$, $p=0.011$ ), for ME and MI, respectively. However, no significance was found for FP between two motor tasks $(p=0.089)$.

\section{Eight-class BCI-menu performance}

The 8-class BCI menu was calculated from the extended experiment on two 
subjects, and compared with the same subjects (see Fig. 5). The TPR of ME and MI was $70 \%$ and $65 \%$ for subject 1 , and $63 \%$ and $50 \%$ for subject 2 , respectively. These results slightly decreased with the increased number of class, for both motor tasks of both subjects. However, the two subjects got a FP of $2.4 / \mathrm{min}$ and $3.2 / \mathrm{min}$ in ME testing, and $2.0 / \mathrm{min}$ and 4.0 /min in MI testing, which were comparable when they were testing with the 4-class menu, without showing a clear trend. Interestingly, the latency was obviously short in the 8-class menu, with a decrease of $0.25 \mathrm{~s}$ and $0.46 \mathrm{~s}$ in ME session, and $0.23 \mathrm{~s}$ and $0.30 \mathrm{~s}$ in MI session, for subject 1 and subject 2,
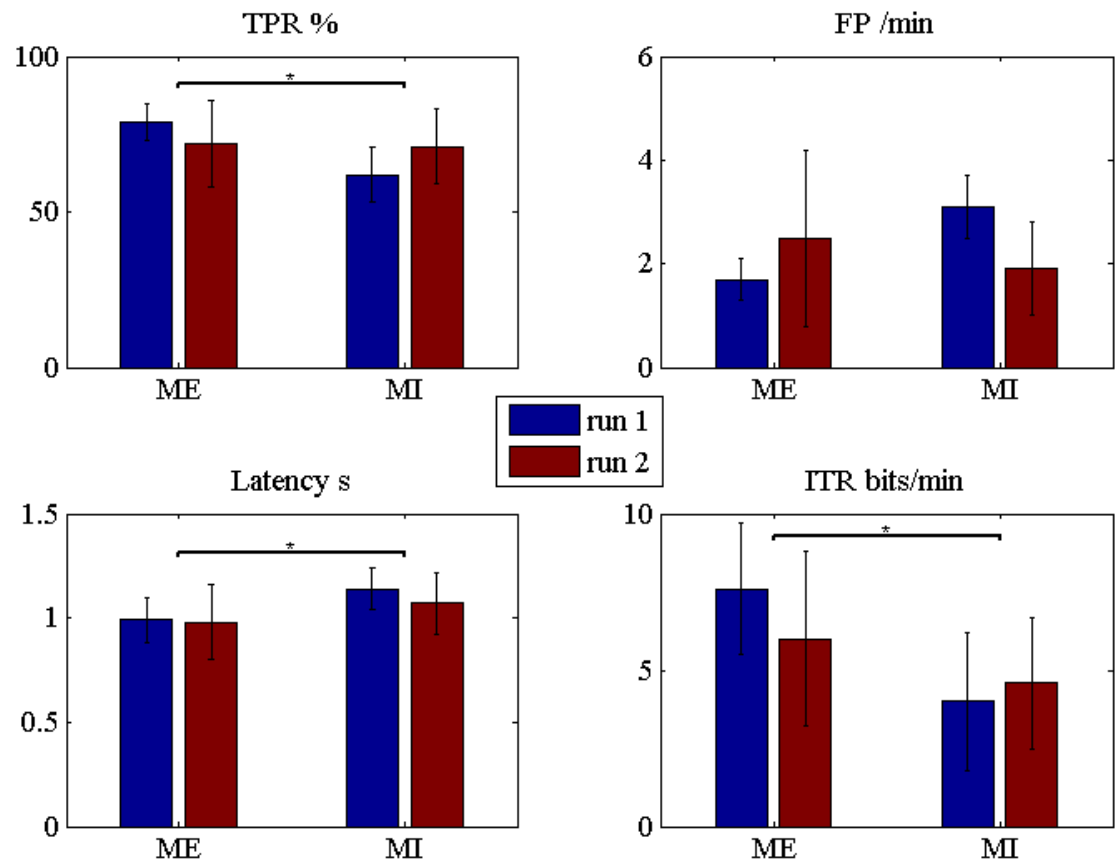

Figure 4.13: Performance of 4-class BCI menu 


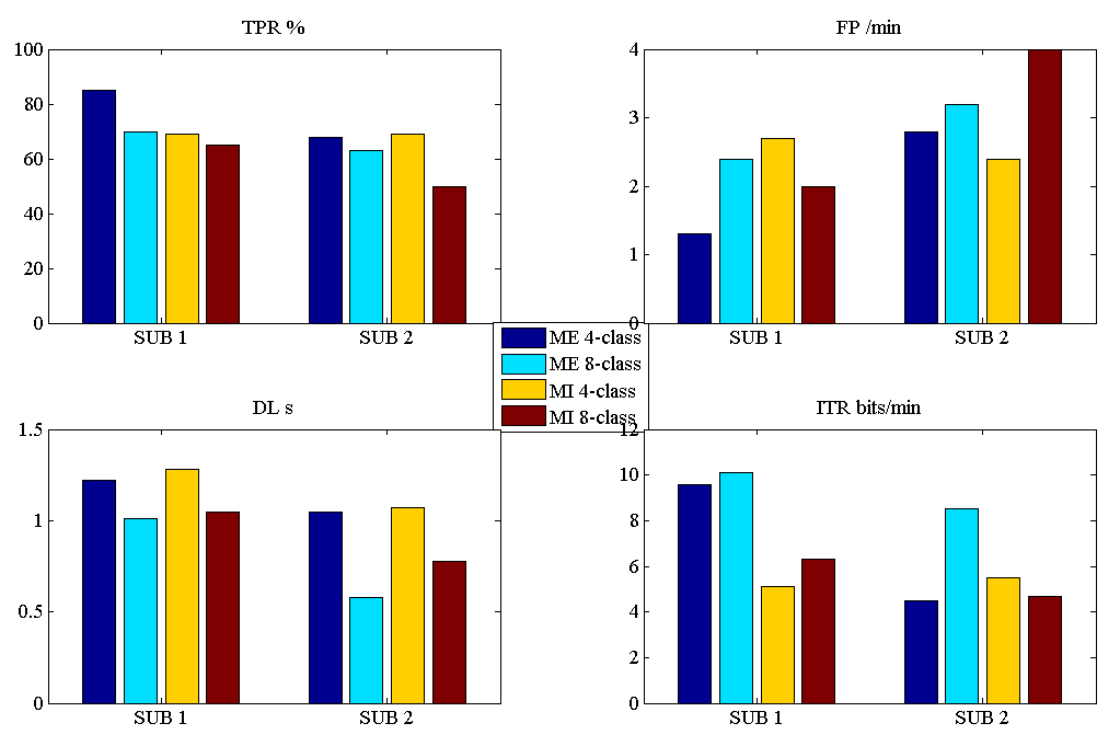

Figure 4.14: Performance of 8-class BCI menu

respectively.

The above changes in TPR, FP and latency resulted in the performance change of ITR. Even though the 8-class menu got lower TPR, due to the increase in class number and decrease in between-class interval, the ITR still tended to increase (increase: $0.4 \mathrm{bits} / \mathrm{min}$ and $1.2 \mathrm{bits} / \mathrm{min}$ for both $\mathrm{ME}$ and MI of subject 1 , and $4.6 \mathrm{bits} / \mathrm{min}$ for ME of subject 2).

\subsubsection{Discussion}

In this study, we proposed a novel multi-class BCI for communication purpose, which was implemented by a special combination of a fast brain switch and an ES menu. The multiple options were provided by the users endoge- 
nous sensory discrimination of an ES loop on the neck, while the choice was intuitively made by a fast brain switch through real-time detection of MRCP from scalp EEG. For the main experiment with 4-class BCI, it yielded a TPR of $\sim 80 \%$ and $\sim 70 \%$, and ITR of $>7 \mathrm{bits} / \mathrm{min}$ and $>5 \mathrm{bits} / \mathrm{min}$, for the ME and MI based brain switch. Further, we extended this BCI to 8-class, and a comparable performance was shown in two subjects, indicating the expansibility of the current system.

\section{Main advantages of the multi-class BCI in the study}

1) Gaze-independant

In the past a few decades, visual speller has been the most investigated BCI based communication system [23][68][49]. In a typical paradigm, visual P300 would be detected when the user fix his/her gaze on the desired symbol, which flashes in an oddball sequence [22]. The past intensive investigations resulted in well-developed P300 speller system, with a large number of class, e.g. 66 matrix [77][21], and a fairly high ITR of up to $40 \mathrm{bits} / \mathrm{min}$ [23]. Some

commercial products are also emerging, such as intendiX [28]. However, the performance would dramatically decrease if the user lost part of the gaze control [14], which is usually the case for patients suffering from vision impairment.

Compared with these visual spellers, a prominent advantage of current BCI is its gaze-independent property. This opens a door for those patients who cannot use the P300 based BCI. For other visual BCIs which do not require 
gaze control, e.g. those use SSVEP [63], the user still needs to focus on the monitor which delivers visual stimuli. This kind of system is thus not optimal for applications such as locomotion through wheelchair control, as it requires the user to shift their focus between the monitor and the surroundings. On the contrary, the monitor is not necessary for our BCI, and thus an ambulatory system would be possible.

There are other gaze-independent BCIs using SSSEP [56] or Tactile P300 [12]. A 4-class tactile BCI was reported with the accuracy of $\sim 60 \%$ and the ITR of $2 \mathrm{bits} / \mathrm{min}$, which is much lower than our results (TPR: 70 80\%, and ITR of 57 bits/minutes). Besides, in these studies, the tactile stimulators were placed on the fingers [56][12], and thus would not be used by high-level paralyzed patients without sensory below neck.

2) Endogenous discrimination

Another group of multi-class BCI is realized by the classification of a number of motor imageries [13], mental tasks [25], or the combination of both [26]. This kind of BCI extremely relies on the classification performance of different EEG patterns, which is a very challenging task due to the poor signal-to-noise ration of EEG. Therefore, it usually requires an intensive subject training, but still limited by the number of class. Still, the performance is low in the reported studies, such as the maximum accuracy of $62 \%$ for a 4-class motor imageries in an offline study [13], and online performance $\sim 50 \%$ for a 4-class BCI with both motor and mental tasks [26]. No ITR was reported in these studies, but the approximate value could be deduced 
according by equation 4.1 and 4.2 , which would result a very small number, given the low accuracy and long period for single trial (8-13 s).

Instead of struggling in the challenging multi-class classification, our BCI system got an alternative solution from the users endogenous discriminant sensory. The systems performance relies directly on the users distinguishing ability in external stimuli, rather than the discriminant EEG patterns induced by these stimuli. That is why the performance of the proposed BCI I superior to those based on classification of multiple EEG patterns. Moreover, for patients who have preserved sensory on upper limbs, e.g. complete locked-in patients, but without discriminant information from the EEG pattern induced by external stimuli [57], our system can still work effectively.

\section{Factors that influence the performance}

Several main factors which may affect the performance of the proposed multiclass BCI, including performance of the brain switch, number of class, and period of ES loop, were discussed as follows.

1) Peformance of the brain switch

The accuracy of the brain switch may directly determine the accuracy of the multi-class BCI, and thus influence the ITR according to equation 4.1. In addition, the detection may contribute to the BCIs latency, which determines the minimal period of ES loop, and thus also affect the ITR (see equation 4.1). Therefore, the performance of the brain switch is the crucial factor for the proposed BCI system. 
In previous studies, as well as this one, we demonstrated the robust brain switch by real-time detection of MRCP. It yielded a TPR $\sim 80 \%$, and more importantly, a latency of $\sim 300 \mathrm{~ms}$ [93]. This ensured the high performance of the multi-class BCI in this study.

Beside MRCP, there is another brain switch using movement related potentials, i.e SMRs, from similar motor imagery. The high accuracy using SMRs was reported in literature, such as Beta ERS detection of TPRs above $80 \%$ [55][88], and the performance of ERD above 70\% [70][98]. However, the detection latency was reported to be several seconds [32].

Therefore, even the SMR based brain switch yielded similar TPR as the one using MRCP, the seconds long latency would obviously extend the time for each time, resulting a dramatically decrease of ITR. Beside, the type of motor task, i.e. ME or MI, affects the TPR, and further influence the BCI performance. As shown in Fig. 4, the ME based BCI got significantly better performance than that with MI. This is mainly due to the better signal-tonoise ratio of MRCP from real movement [19], which resulted in a improved performance of the brain switch.

Actually, the second MI testing session is even more relevant to real applications on patients, who could barely perform real movement and thus the motor imagery is the only output. However, as there is no direct reference for self-paced imagination, the objective measurement of performance is difficult. As such, the first testing session is also essential for system verification, where the timing of real movements were recorded with EMG. 


\section{2) Period of ES loop}

The period of ES loop is another key factor that may contribute to the performance of the current BCI. The decrease of this period would reduce the transfer time for each symbol, and according to equation (2), resulting the increase of ITR.

This period includes two parts: an ES stimulation interval $(0.2 \mathrm{~s}$ in this study), and an inter-stimulation interval. In a pilot study, the discriminant sensory was found to be weaken when the stimulation interval was set to less than $0.2 \mathrm{~s}$. Given the current short interval $(0.2 \mathrm{~s})$, there is not so much space for improvement. On the other hand, the optimizing of inter-stimulation interval might be of more practical value. We should notice that the interval, e.g. 1.8 in 4-class BCI, is used for the user to trigger the brain switch. Therefore, it should be longer enough for the user to make such a choice (i.e. dorsiflexion) which can be detected by the brain switch. Otherwise if this inter-stimulation interval was too short, false positives may increase due to the delayed dorsiflexion or the delayed detection of such movement or imagery. This would lead to a decrease of TPR, and further reduce the ITR. Based on the above analysis, we know that the inter-stimulation, which depends on the BCIs latency, should be taken carefully to reach an optimal solution. As indicated in Section 2.5.1, this latency came from the users response time and the detection latency of the brain switch. The improvement in the latency of the brain switch would definitely improve the BCI performance, but it would require a superior algorithm. For the response time, the 
users training may contribute to a significant decrease. Since we found that the latency was short than $1.5 \mathrm{~s}$ in the 4-class BCI, we set the ES period to this value in the 8-class BCI. And interestingly, this latency further reduced to $<1 \mathrm{~s}$, indicating the effect of subject training.

3) Number of class

In this study, we started with a 4-class BCI in the main experiment. Fourclass BCI has a large number of potential applications, such as communication command of YES/NO/PASS/END for clocked-patients, and direction for wheelchair control. However, the performance of non-invasive BCI with four classes, especially for those gaze-independent, still needs further improvement. The main reason is that, as discussed above, the multi-class classification problem was limited by the quality of EEG signal. As a first step of the current setup, we demonstrated a 4-class BCI with a TPR up to $80 \%$, and ITR $>7$ bits/min. Further, we showed the concept of a 8-class BCI, whose ITR appears to be even higher. This gives us a hint for the possibility of increased performance with a large number of classes, which would be a big step towards real applications.

\subsection{Summary}

In this Chapter, we proposed two closed-loop BCI systems based on the short-latency brain switch. In the first system, the brain switch was used to trigger a MAFO in real-time, which induced passive dorsiflexion immedi- 
ately, providing timely feedback to the brain. Such a self-paced system was demonstrated to be able to induce neuroplasticity on healthy subjects with a very short intervention, indicating a promising tool for stroke rehabilitation. The second system was implemented by combining the brain switch with a tactile menu, resulting in a multi-class BCI. The concept of this novel system was intensively tested on healthy subjects, where its desired properties, such as gaze-Independence and high ITR, were fully demonstrated. Based on these results, the application of this system on communication and motor control is feasible. 


\section{Chapter 5}

\section{Clinical measurement}

In previous chapters, we intensively investigated the short-latency BCIs on healthy subjects, which could be potentially used by paralyzed patients. Before large scale clinical studies, a pre-clinical measurement was performed on SCI patients in this chapter, to verify the MRCP characters in this patient group.

This Chapter was modified from a first-author publication in an open-access journal [97].

\subsection{MRCP in paraplegic patients}

\subsubsection{Motivation}

MRCPs are influenced by impairments of the sensory-motor system. Castro et al. [16] compared MRCPs in three subject groups: healthy individuals 
who executed movement of the left and right leg, healthy subjects who only prepared for the same movements, and chronic complete SCI patients who imagined the same movements. They observed that the amplitudes of the readiness potential and motor potential were lower in SCI patients than in healthy subject executing the movement, but were comparable between SCI patients and healthy participants who only prepared for the movements. All SCI patients had a complete injury with no preserved sensation under the level of the injury, thus in that study it was not possible to distinguish the effects of sensory and motor loss, specifically complete/incomplete injury in the sensory pathways. Therefore, our first research question is related to the role of sensory information, which can be investigated in complete/incomplete SCI subgroups.

A frequently overlooked co-morbidity of paralysis is Central Neuropathic Pain (CNP), present in $40 \%$ of the SCI population, equally affecting paraplegic and tetraplegic patients with complete or incomplete injury [79]. CNP is a consequence of an injury to the somato-sensory system [30], and as such it originates at the cortical level. Functional magnetic resonance imaging (fMRI) studies showed that this type of pain modulates the activity of the motor cortex [29] of both paralyzed painful limbs and non-paralyzed limbs. In a recent study, Vuckovic et al. [86] compared the ERD/ERS [66] in patients with paraplegia and CNP, patients with paraplegia and no pain and healthy individuals with no pain. Patients with CNP had strongest ERD in the theta, alpha and beta bands, while ERD was less expressed in healthy 
participants. Patients with no pain had the weakest ERD. However, it is not clear if the presence of CNP would equally affect MRCP and ERD/ERS, as it is believed that the two signal modalities have different origins [5][66]. This raises our second research question: the role of pain in MRCPs of SCI patients, which can be studied in pain/no-pain SCI subgroups. The role of abnormality patterns in MRCP is relevant in BCI applications. In previous chapters, two BCI systems based on MRCP were proposed and tested on healthy subjects, for rehabilitation and communication purpose, respectively. The MRCP was used in these systems as a trigger signal (brain switch) to control an external device, such as an active orthosis or electrical stimulator. SCI patients are ideal candidates for the proposed rehabilitation and communication systems. Especially those patients with incomplete injury might benefit from a combined sensory-motor therapy, as the one proposed by using brain switch driven mechanical stimulation. However, the characteristics of MRCPs in these patients are not known yet and this information is relevant for the design of a detector based on MRCP waveforms. Hence, the implication on potential applications of BCI for SCI patients is the third research question we will address.

This study presents the initial step in developing an MRCP-based BCI system for SCI patients. For this purpose, we investigated the difference in MRCP morphology between SCI patients and healthy subjects, as well as the unique features of MRCP in sub-groups of patients with different degrees of CNP and scale of impairment (complete or incomplete paralysis). Further, 
the related issues for BCI application are discussed.

\subsubsection{Subjects and experiment}

\section{Subjects}

Eight healthy volunteers (HV) and 14 SCI patients, with either complete or incomplete paralysis, participated in this study. The neurological level of injury was determined using the American Spinal Injury Association (ASIA) Impairment Classification [50]. SCI patients were further classified on the basis of presence or absence of CNP, below the level of the injury. Inclusion criteria for patients with pain (PWP) was that they were at least one year post-injury, were treated for CNP for at least 6 months, had a pain level 5 on the Visual Numerical Scale (VNS) and had the injury at level T1 or lower. Inclusion criteria for patients with no pain (PNP) were that they were at least one year post-injury, with injury at level T1 or lower. General exclusion criteria for all three groups were age under 18 or over 55, existence of any other chronic or acute pain at the time of the experiment, brain injury or other known brain condition that would influence EEG interpretation or would prevent the patients from understanding the experimental task. Details about the subjects self-reported information are presented in Table 1. 


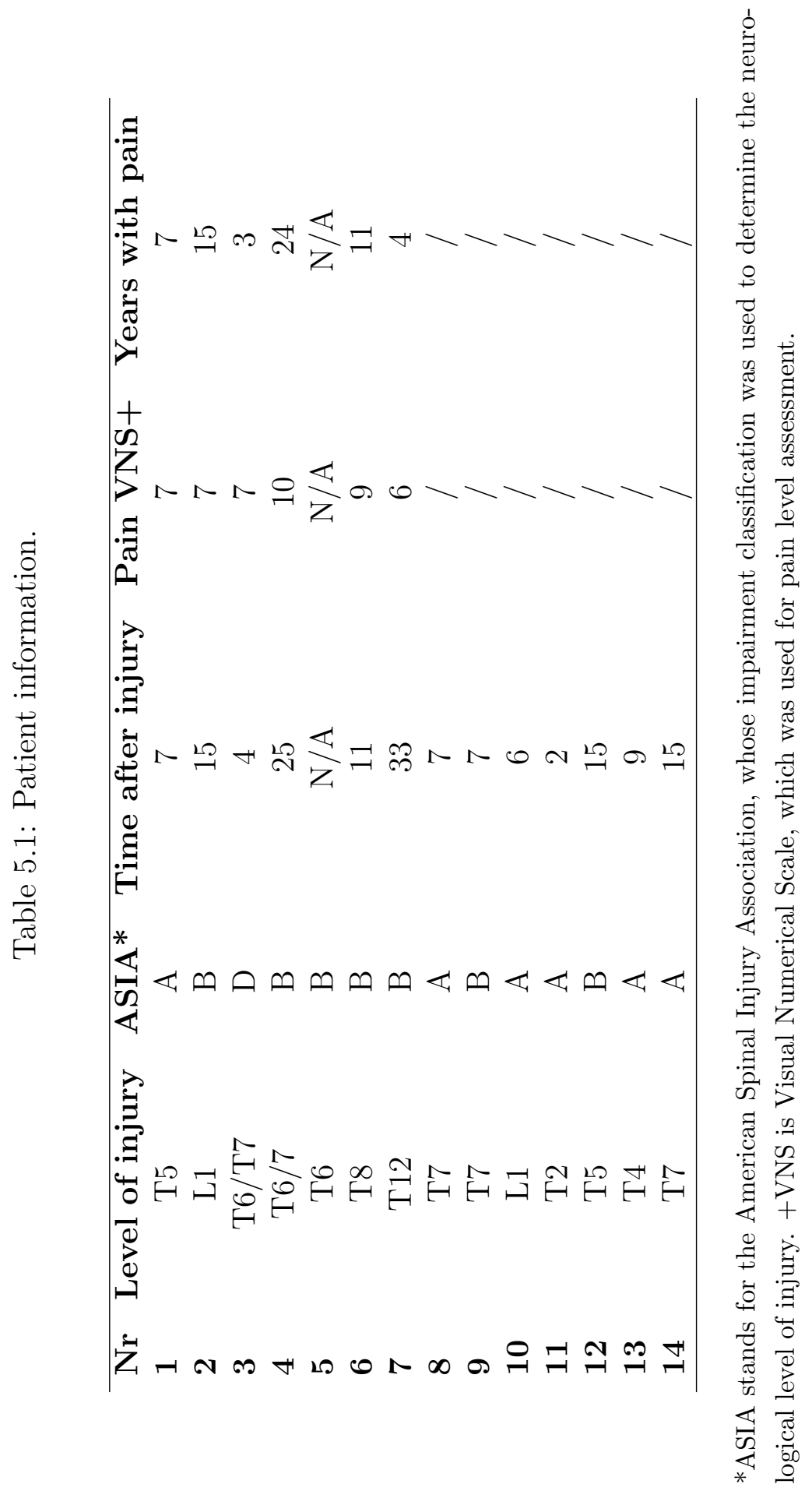




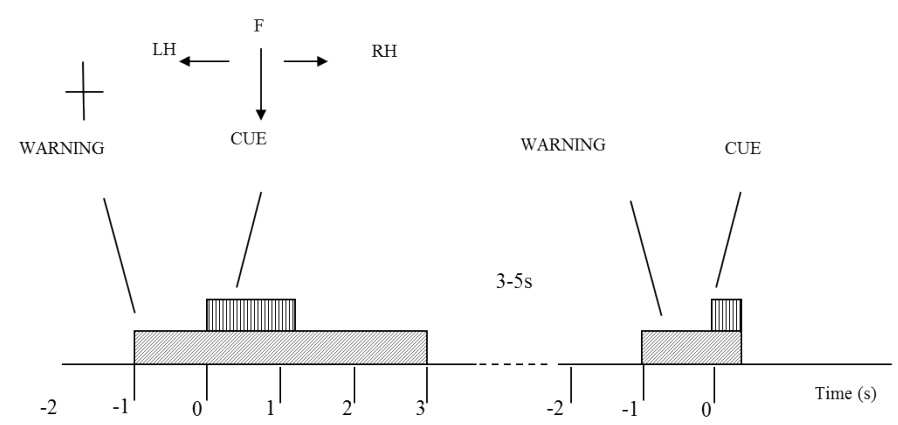

Figure 5.1: Clinical experiment protocol. At $t=-1$ a cross appears on the screen, indicating the participant should prepare for a motor imagery. One second later, an arrow pointing to right, left or down, and the participant were asked to perform a motor imagery of their right hand $(\mathrm{RH})$, left hand $(\mathrm{LH})$ or feet $(\mathrm{F})$ accordingly, till the cross disappear at $\mathrm{t}=3 \mathrm{~s}$. Afterwards there is a rest period of $3-5 \mathrm{~s}$, before the next trial begins.

Informed consent was obtained from all participants, and the study protocol was approved by the University of Strathclyde Ethical Committee for the HV group and the National Health Service for Greater Glasgow and Clyde Ethical Committee for the SCI group.

\section{Experiment protocol}

The participants were comfortably seated at a desk, at a distance of approximately $1.5 \mathrm{~m}$ from the computer screen on which visual instructions were provided. They were instructed to look at the center of the screen and to perform motor imagery following visual cues while minimizing eye movements. For each trial, at $\mathrm{t}=-1 \mathrm{~s}$ a readiness cue (a cross + ) appeared and 
would remain for $4 \mathrm{~s}$ (Figure 5.1). At $\mathrm{t}=0 \mathrm{~s}$ an initiation cue, presented as an arrow, appeared next to the cross sign, for a duration of $1.25 \mathrm{~s}$. The arrow pointed either to the left, right or down, corresponding to the motor imagery tasks of left hand waving ( $\mathrm{LH})$, right hand waving $(\mathrm{RH})$ and tapping with both feet $(\mathrm{F})$, respectively. The participants were asked to continue to perform imaginary movements until the cross disappeared from the screen $(3 \mathrm{~s}$ after the initiation cue appeared). In total, 60 trials of each of the three type of motor imagery were performed by each subject in one session. The trials were divided in groups of 10 for each type of imaginary movement (LH, RH and F). Sequences of motor imagery tasks appeared in a random order and at random 3-5 s intervals.

We instructed the participants to perform motor imagery, and we asked them specifically to imagine, not to attempt movements. However, it should be noted that while HV practiced motor imagery, SCI patients might have also attempted to move their paralyzed limb.

A 61-channel EEG recording was performed with an EEG device (Synamps2, Neuroscan, USA). The electrodes were placed according to the standard 1010 locations. EOG was recorded from 3 channels around the right eye. EEG and EOG were recorded with respect to the linked ear reference and the ground was at $\mathrm{AFz}$. In addition, EMGs were recorded from the right and the left wrist extensor muscles and right foot dorsiflexor using the bipolar inputs to the Synamps device. The purpose of EMG recording was to check for the absence of any voluntary movements when the subjects attempted motor 
imagery. The sampling frequency was $1000 \mathrm{~Hz}$. The electrode impedance was kept below $5 \mathrm{k} \Omega$ during all measurements.

\subsubsection{MRCP morphology in paraplegic patients}

\section{Signal pre-processing}

The EEG signal was down-sampled to $250 \mathrm{~Hz}$ and pre-processed with a bandpass filter at $0.1-3 \mathrm{~Hz}$ (second order butter-worth), followed by the large Laplacian filter of the respective central channels $(\mathrm{Cz}$ and $\mathrm{C} 3$ for foot and right hand, respectively) and eight second-nearest channels around them $(\mathrm{Fz}$, FC1, FC2, C3, C4, CP1, CP2, and Pz for foot, and F3, FC5, FC1, T7, Cz, CP5, CP1, and P3 for right hand, respectively). This was done to reject the

common mode noise and thus increase the signal-to-noise ratio [51][59]. For each trial, the segments between $\mathrm{t}=-2 \mathrm{~s}$ and $\mathrm{t}=6 \mathrm{~s}$, with respect to the cue onset, were extracted as MRCP. All trials were visually inspected to reject trials that were potentially corrupted by artifacts and noise. All trials were of good quality and no trial needs to be rejected.

\section{Statistical analysis of MRCP morphology across subject groups}

We performed the following three pair-wise analyses:

1. HV vs SCI: healthy volunteers vs all SCI patients independent of the level of injury or the presence of pain (8 HV vs $14 \mathrm{SCI}$ );

2. PNP vs PWP: SCI patients with no pain vs SCI patients with CNP (7 PNP vs 7 PWP). Both PNP and PWP group contained patients with com- 
plete and incomplete injury;

3. CP vs IP: SCI patients with complete injury (ASIA A complete loss of motor and sensory functions under the level of the injury) vs SCI patients with incomplete injury (ASIA B/C/D with some sensations preserved under the level of the injury), (6 CP vs 8 IP). Both CP and IP groups contained patients with or without pain.

In order to compare MRCP morphology between groups, a Wilcoxon ranksum test was utilized for statistical analysis. Three comparisons were performed for each type of movement imagery. The null hypothesis was that, for each type of movement within each group, the MRCPs have the same average value at the same temporal location. For each comparison, the entire $8 \mathrm{~s}$ long interval was divided into $0.1 \mathrm{~s}$ long segments and statistical analysis was performed between groups separately for the 80 segments of each case. The statistical significance level was set to 0.05, with a Holm-Boniferroni correction [36] applied (smallest p-value was 0.05/80).

\section{Results}

The MRCPs of foot imagery are compared for the subject groups (i.e. HV vs SCI, PNP vs PWP, and CP vs IP) in Figure 5.2. The amount of MRCP segments is equal to the product of number of trials and number of subjects. As stated in Section 5.1.2, the number of trials is 60 for each type of task by each subject. E.g. we had $8 \mathrm{HV}$ and 14 SCI, the amount of segments for HV and SCI is 480 and 840, respectively. The difference is particularly 
pronounced for the case of HV vs SCI. In general, the amplitude of MRCP for the SCI group was significantly greater than that for the HV group (peakto-peak value: $5.6 \pm 6.3 \mathrm{~V}$ vs $2.7 \pm 3.4 \mathrm{~V}$ ). The $\mathrm{CP}$ groups MRCP amplitude was also slightly greater than the IP group, while there was only a small difference in amplitude for PNP vs PWP. The evoked responses following the readiness cue (the ' + ' sign) and the initiation cue (arrows) are clearly visible in all cases.

Period of general preparation for movement: During the period $t=-1 \mathrm{~s}$ to $\mathrm{t}=0$ $\mathrm{s}$, i.e. after the + appeared and before the arrow appeared, SCI subjects had a significantly larger positivity than HV subjects. This significant difference lasted until $-0.7 \mathrm{~s}$, i.e. $300 \mathrm{~ms}$ after the appearance of the '+' sign. This is an indication of altered (enhanced) response to a movement related visual cue from the SCI patients. A significant difference was found for the CP vs IP group: CP patients had larger visual-motor positivity than IP subjects. This result suggested that complete loss of sensory information (CP group) from the foot enhanced the potential, compare to subjects with some remaining sensory input (IP group). These results imply that the level of deafferentation is positively related to the magnitude of visual evoked potentials. On the other hand, no statistical significance was found in the PWP vs PNP group, indicating that presence of pain does not affect the magnitude of the preparation potentials.

The visual-motor potential from motor imagery of the hand at C3 during this period (Figure 5.3) was smaller in magnitude compared with that of foot im- 
agery at Cz. For HV vs SCI groups, similar to foot imagery, a difference was found around $-0.7 \mathrm{~s}$. The difference did not reach statistical significance, probably because it was much smaller both in amplitude and duration than in the case of foot imagery. No statistically significant difference was observed for the CP vs IP group. Similar to the case of foot imagery, no significant difference was found for PNP vs PWP group.

A difference at $\mathrm{Cz}$ between $\mathrm{HV}$ and PWP is because SCI had stronger positivity at around $\mathrm{t}=-0.7$ at $\mathrm{Cz}$ (painful limbs) than they had at $\mathrm{C} 3$ which corresponds to a non-paralyzed limb. It is true that we showed $\mathrm{Cz}$ for $\mathrm{MI}$ of legs and C3 for MI of right hand, but we also checked that SCI has larger positivity at $\mathrm{Cz}$ than $\mathrm{HV}$ even for motor imagery of hands. For $\mathrm{HV}$ there does not seem to be a difference between $\mathrm{Cz}$ and $\mathrm{C} 3$ in a period $\mathrm{t}=-1 \mathrm{~s}$ to $\mathrm{t}=0 \mathrm{~s}$.

In summary, during this period of general preparation for movement, the subjects did not know what type of motor imagery should be performed, so the visual-motor potential was not task-specific. Therefore, the consistent results between foot imagery and hand imagery, other than the overall magnitude difference, are expected. This is particularly the case for HV vs SCI group, where significance was detected at this point for foot imagery. While for hand imagery a distinct peak of the p-value existed, no statistical significance could be established.

Period of movement specific-preparation and covert motor execution: As presented in Figure 5.2, during the period of $t=0 \mathrm{~s}$ to $\mathrm{t}=3 \mathrm{~s}$ of foot motor 
imagery, a statistically significant difference can be noticed in all three pairs of comparisons. The largest difference was observed between HV and SCI. SCI patients had significantly larger amplitude of the positive peak at 300 ms. The MRCP negativity of the SCI group was also significantly larger than for the HV subjects. The rebound from the negativity of the HV group appeared around $t=1 \mathrm{~s}$ and then returned back to baseline around $t=3 \mathrm{~s}$; for the SCI group, the rebound was more gradual, reaching the baseline at approximately $\mathrm{t}=6 \mathrm{~s}$ without a second positive peak. The main difference between PNP group and PWP group was located between $t=0 \mathrm{~s}$ and $\mathrm{t}=1$ s, where descending for the PWP group was faster than that for the PNP group. Similarly the largest difference between CP and IP group could be noticed in the first $0.5 \mathrm{~s}$ following the directional cue. The CP group presented higher amplitude of the positive peak and faster decreasing slope than IP group.

For hand imagery, the differences after the initialization cue were much smaller than difference for feet imagery for all groups. This is expected since none of the subjects had sensory or motor impairments of the upper extremities. Still, there was a statistically significant difference between HV and SCI groups in part of the rebound phase (from $1.2 \mathrm{~s}$ to $2.3 \mathrm{~s}$ ). However, there is no statistical difference between PNP and PWP group and between CP and IP group.

In summary, largest differences during the period following the directional cue were noticed, as expected, between HV and SCI group and they were 

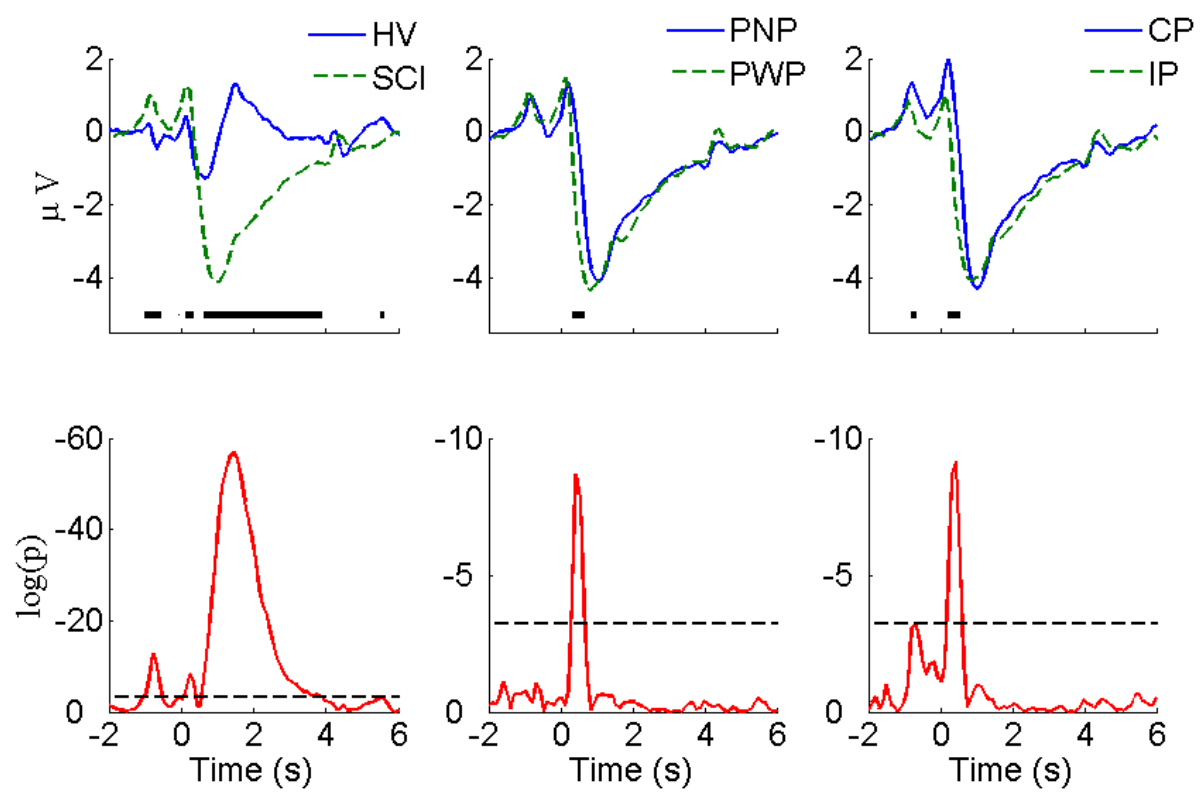

Figure 5.2: MRCP morphology and statistical comparison for large Laplacian $\mathrm{Cz}$ (motor imagery of foot). The figures in the upper row present the average MRCP from different groups (HV vs SCI, PNP vs PP, and CP vs IP). The thick horizontal lines indicate the portions in which statistically significant difference was detected using the Wilcoxon rank-sum test with Holm-Boniferroni correction. The p-values of the statistical tests were presented in the lower row, with logarithm scale (logp). The dashed horizontal line indicates the minimal significance level in the Holm-Bnifeernoi correction procedure, i.e. $\log (0.05 / 80)$, since there were 80 simultaneous tests in each case. 

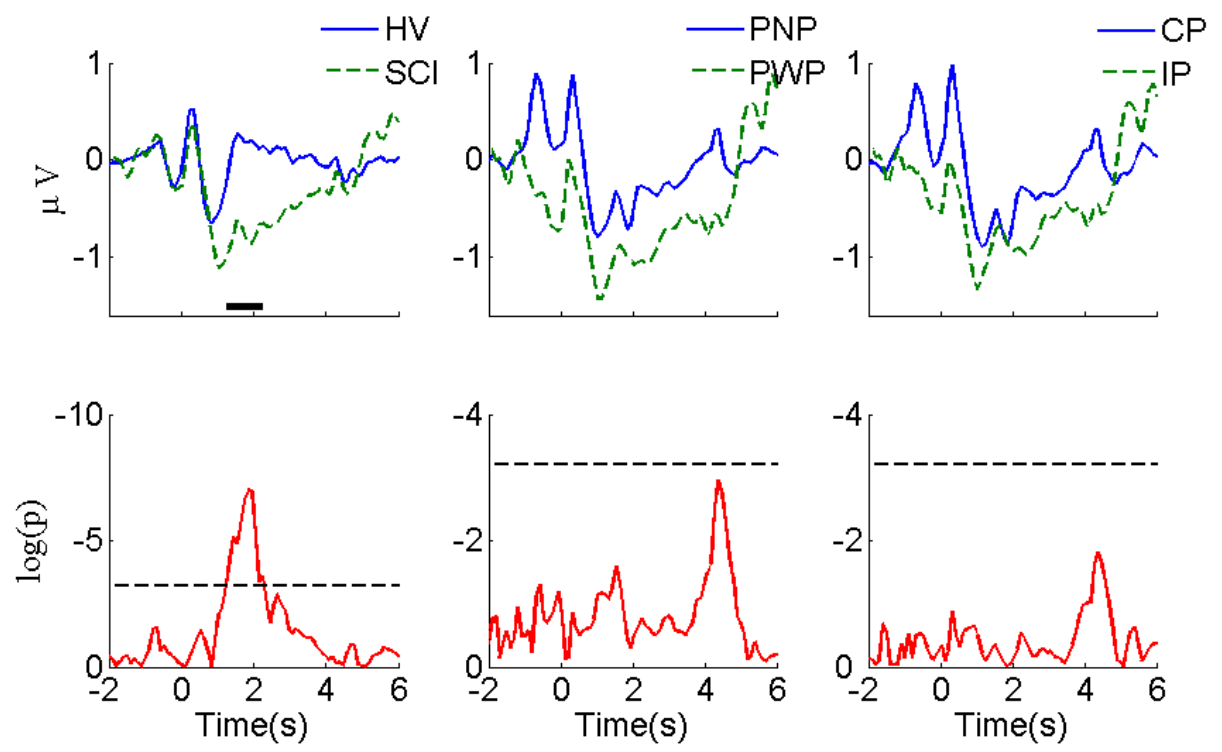

Figure 5.3: MRCP morphology and statistical comparison for large Laplacian C3 (motor imagery of right hand). The convention and legends are the same as in Figure 5.2.

present for both paralyzed and non-paralyzed limbs. Smaller differences, both in magnitude and duration, also existed between PWP and PNP group and for CP and IP group, for motor imagery of feet. However no statistically significant difference was observed for motor imagery of right hand in either of the patient sub-groups.

\subsubsection{Classification of MRCP}

\section{Classification method}

Following the above statistical analysis, a two-class classification was performed on MRCPs of each task (feet and right hand) corresponding to the 
three pairs defined above, i.e. HV vs SCI, PNP vs PWP, and CP vs IP. The classification was performed with a dimensionality reduction algorithm called locality persevering projection (LPP) [33][34], followed by a k-nearestneighborhood $(\mathrm{kNN})$ classifier. LPP, a manifold-based method, was demonstrated to be superior than linear methods such as PCA and LDA when data have clear nonlinear characteristics [34]. LPP can preserve the data structure in the original manifold when projecting data into lower linear feature space, of which classic linear dimensional reduction algorithm such as PCA or SVD is not capable. It was previously used for MRCP detection, in which it outperformed linear match filter method [93]. A 5-fold cross-validation was used to validate the classification accuracy. The classifier was trained with randomly selected $4 / 5$ of single-trial MRCPs and the remaining $1 / 5$ were considered as testing sets. The LPP algorithm was used to project the training samples into a lower dimensional space, while preserving its intrinsic structure in its original manifold, as in [93]. The reduced dimension was chosen as $60 \%$ of the original data dimension, which was proved to be optimal for MRCP detection [93]. Next, the projected data in this LPP subspace were used to train the classifier. In the subsequent testing step, testing samples were projected into the LPP sub-space obtained through training, which was then classified using the trained $\mathrm{kNN}$ into either class of the pair (e.g., HV or SCI). The classification performance was quantified with the classification accuracy, i.e. the percentage of correctly classified trials with respect to the total number of testing trials. 
Aiming at investigating the temporal discriminant information in the MRCPs among different groups, the classification was not performed on the entire MRCP segments, but with processing window of segments at different temporal location as well as with different segment lengths. This process was done by sliding the starting point of the processing window, from $t=-2$ $\mathrm{s}$ to $\mathrm{t}=3 \mathrm{~s}$ ( step size $0.1 \mathrm{~s}$ ). At each starting point, the length of the window also changed from $1 \mathrm{~s}$ to $3 \mathrm{~s}$ (step size $0.1 \mathrm{~s}$ ). As the movement imagery was performed until $\mathrm{t}=3 \mathrm{~s}$, it was not practically useful to process signals $3 \mathrm{~s}$ after the movement onset.

\section{Classification performance}

Figure 5.4 illustrates the classification accuracies of the three groups, as a function of the starting point and the length of the processing windows. It was possible to classify between foot imagery of the HV and SCI group with higher accuracy than the two patient sub-groups (Figure 5.4). This is in accordance with the largest statistical difference found between the MRCP of HV vs SCI, as presented in Figure 5.2.

The highest average accuracy of HV vs SCI was $90.5 \%$ (at window start=-1 s and window length=3 s), while those of PNP vs PWP and CP vs IP were 68.7 $\%$ (at window start=-0.1 s and window length=1.4 s) and $65.1 \%$ (at window start $=0.2 \mathrm{~s}$ and window length $=2 \mathrm{~s}$ ), respectively. In addition, the accuracies changed according to the window start and length, and the patterns of this change are different among the three pairwise groups. For HV vs SCI, the 

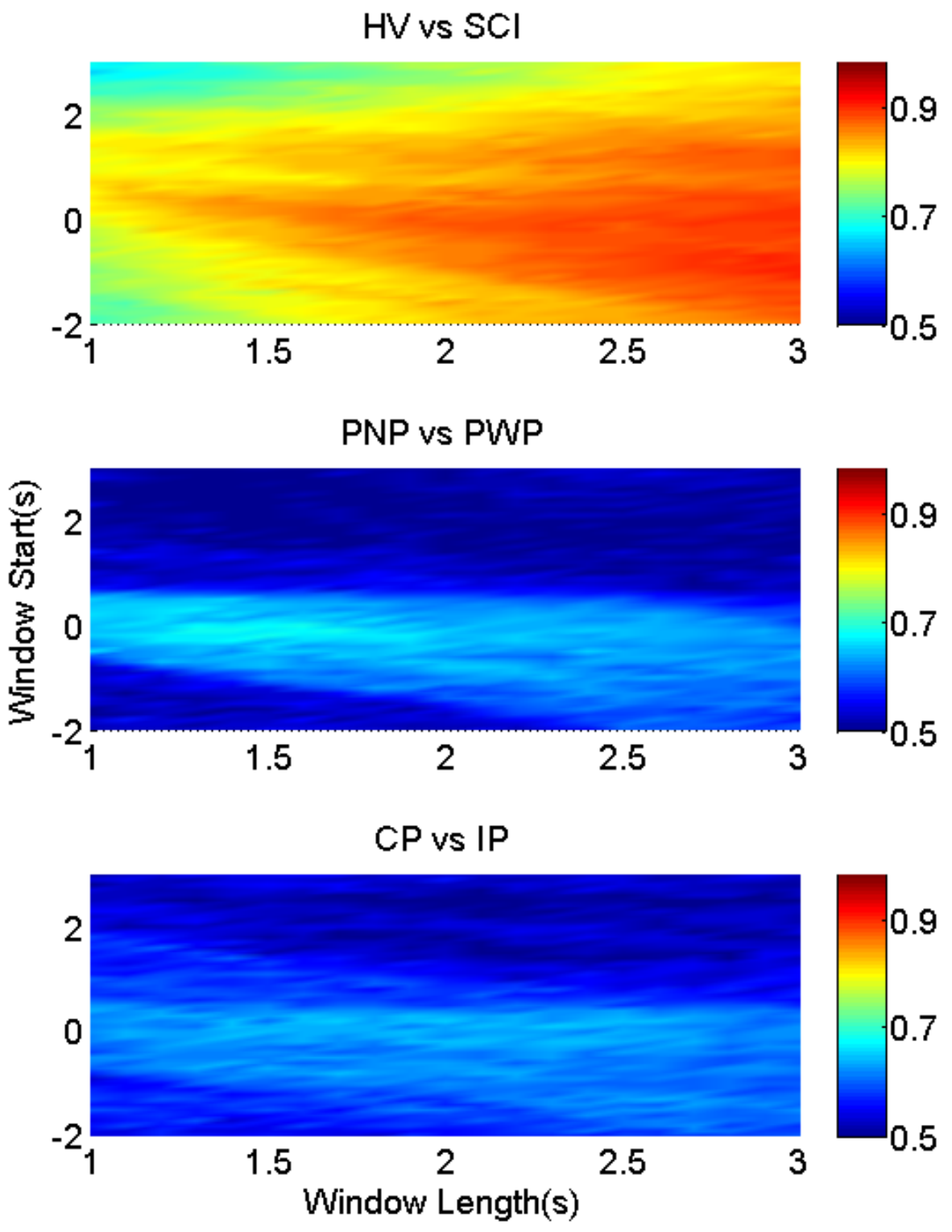

Figure 5.4: Classification accuracy for foot imagery. The color-bars identify the classification accuracies of MRCP segments from three pairwise groups, with onset from $t=-2 \mathrm{~s}$ to $t=3 \mathrm{~s}$, and length between $1 \mathrm{~s}$ and $3 \mathrm{~s}$. These three figures from upper to bottom represent the results of HV vs SCI, PNP vs PWP, and CP vs IP, respectively. 

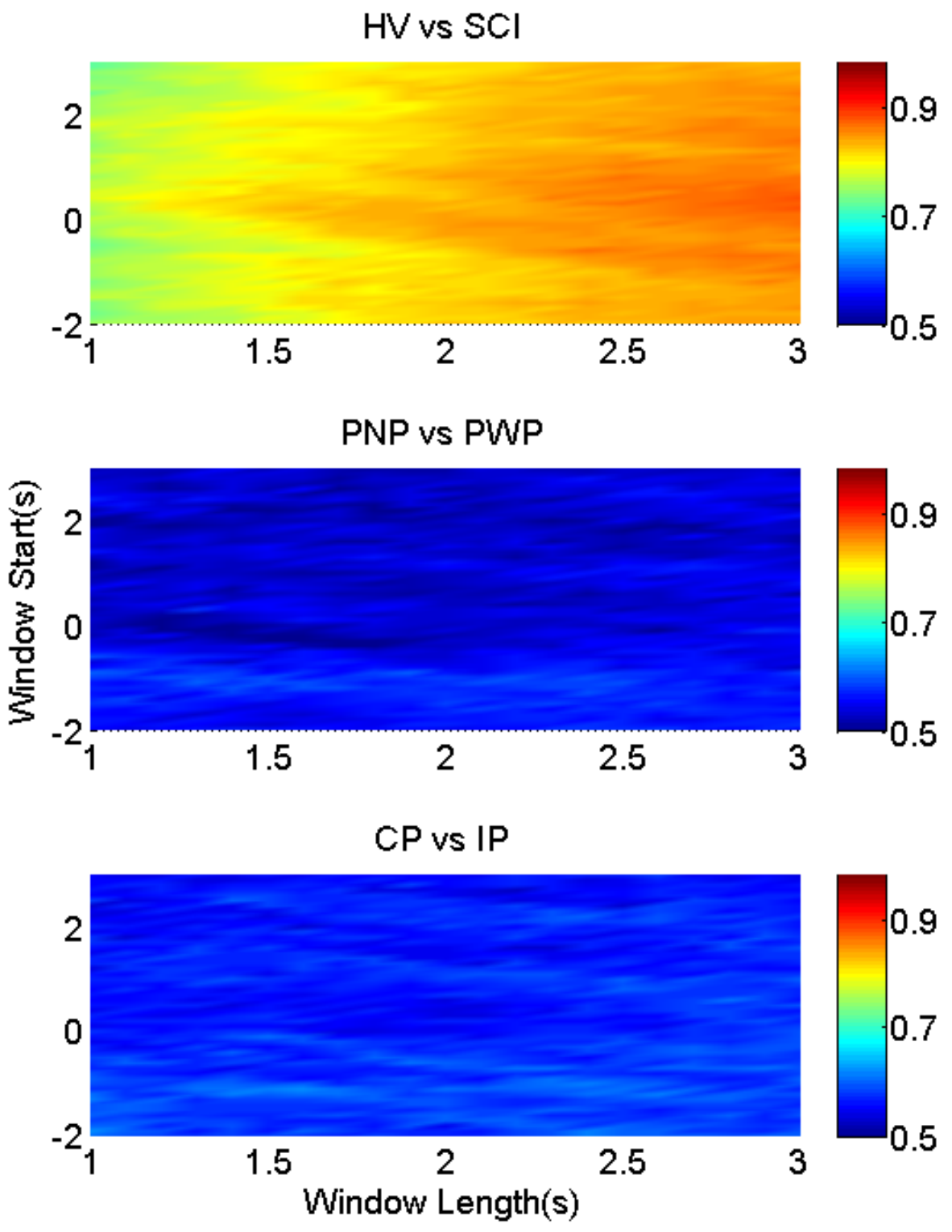

Figure 5.5: Classification accuracy for right hand. The color-bars identify the classification accuracies of MRCP segments from three pairwise groups, with onset from $t=-2 \mathrm{~s}$ to $\mathrm{t}=3 \mathrm{~s}$, and length between $1 \mathrm{~s}$ and $3 \mathrm{~s}$. These three figures from upper to bottom represent the results of HV vs SCI, PNP vs PWP, and CP vs IP, respectively. 
part with accuracies $\sim 90 \%$ was located in the bottom right corner, where the starting point was mostly before $t=0 \mathrm{~s}$, and the length was larger than 2.5 s. High classification accuracies (74\%) were achieved even when only a $1 \mathrm{~s}$ period of general preparation $(t=-1 \mathrm{~s}$ till $\mathrm{t}=0 \mathrm{~s})$ was used to classify between the two groups. As the analysis time window moved towards the movement specific period, shorter windows were sufficient to achieve high classification accuracy, indicating largest difference between HV and SCI during the period of task specific motor imagery. This high classification performance resulted from the large difference in MRCPs between HV and SCI, as shown in Figure 5.4 .

Similar observation holds for motor imagery of the right hand (Figure 5.5), indicating a general influence of paralysis on the signal characteristics. This indicates that paralysis globally changes preparation of movement, not restricted to the paralyzed limb. Nevertheless, the distributions of accuracies for the other two groups are notably different. For imaginary movement of feet the higher accuracy $(>65 \%)$ part for PNP vs PWP were limited in a small strip around window onset $t=0 \mathrm{~s}$, with window length of $1.2 \mathrm{~s}-1.7$ s. This strip with higher classification accuracy exactly matched the MRCP range with lower p values, and higher statistical significance in Figure 5.2. These results indicate statistically significant difference between these two groups in a period of general preparation and in the period of the early preparation/initiation of the covert movement.

The area with accuracies higher than $60 \%$ for CP vs IP was also small, 
but the shape was evidently different from that of PNP vs PWP. It was an approximately horizontal strip where the onset was around $t=0 \mathrm{~s}$ and the length ranges from $1.5 \mathrm{~s}$ to $2.5 \mathrm{~s}$. This shows that preserved sensation do not considerably influence MRCP in the general preparation of movement but it does influence preparation for specific movement of a part of the body with preserved sensation vs part of the body with no sensation.

The classification rate between PNP and PWP in motor imagery of the right hand could reach $50 \%$ only if the period of general preparation was included in the analysis. This indicates that the presence of CNP influences the general preparation of movement in the painful/non-painful and paralyzed/nonparalyzed limb. In a study on the ERS/ERD of the same group of patients [86], a generalized influence of pain on movement of painful and non-painful limbs was also found.

For right hand between CP and IP group, classification accuracy was slightly higher for the period of general preparation, but it has no clear pattern anywhere else.

\subsubsection{Discussion}

This study presented analysis of the difference in MRCP morphology of covert movement between healthy volunteers and patients with spinal cord injury, through direct statistical comparison and through pattern classification. This has implications on performance of BCI control systems based on MRCP which has mostly been tested on healthy individuals. 
The aim of this study was to compare MRCP between healthy volunteers and patients during both general and movement specific preparation, therefore a period of general focus (general non-specific movement preparation) was also taken into account.

While healthy volunteers presented a relatively homogeneous group, the situation of chronic paraplegic patient is more complex. In the current study, we further categorized the SCI patient volunteers into two sub-groups, based on: the severity of paralysis and presence of chronic CNP pain. By combining patients with respect to different criteria, we investigated the influence of loss of motor control ( $\mathrm{HV}$ vs SCI), loss of sensation (CP/IP), and presence of CNP.

\section{Distinction Between HV and SCI groups}

The largest differences in MRCP morphology were found between healthy and general mixed group of paraplegic patients during covert movements of feet in all phases of MRCP. It is interesting that significant difference was found even during the period of visual stimuli. Presenting a general warning sign produced significant difference between the groups (with a peak around 300-400 ms post-stimuli). This can be explained by the combined visual-motor nature of this potential, especially as the motor area is heavily

involved in their generation but do not show much sensitivity to motor task parameters at this specific positive peak [84]. It may be speculated that this positivity is generated by the increased firing rate of cortical neurons 
in motor areas, as in similar instruction delay experiments on primates that showed comparable delays after the cues [17]. Higher amplitude of the peak in SCI patients might be possibly related to higher effort/concentration in SCI patients expecting to imagine/attempt movement of a paralyzed limb. In SCI patients a motor potential in period $\mathrm{t}>0 \mathrm{~s}$ had significantly higher negative peak with a rebound potential also called reafferent potential [16]being delayed for several seconds. The amplitude of the rebound potential was also much lower in SCI group, which is explained by its relation to the kinesthetic feedback.

Statistically significant differences in MRCP morphology between these two groups were also found for motor imagery of the right hand, over electrode location C3, though to a smaller extent. This demonstrated the global influence of paralysis on modified EEG responses, and is in accordance with

previous studies looking into either spontaneous [83][10][86] or evoked brain activity [86] in SCI patients. While the larger negativity during imagination of movement in paraplegic patients resembled the study by Lacourse et al., [43], there were many detailed differences that may originate from different cue type or EEG referencing.

\section{Distinction between SCI subgroups}

The analyzed group of patients was mixed with respect to the severity of paralysis and presence of chronic pain, therefore the results could not be conclusive. Therefore we further compared MRCP in patients with and without 
CNP. CNP is known to affects the activity of the motor cortex [86], thus potentially influencing the morphology of MRCPs. Assuming that CNP is unrelated to the completeness of injury, patients with complete and incomplete injury were mixed. Analysis showed much smaller difference between patients with and without pain than between healthy and general SCI population.

1) The Effect of CNP

It is known that CNP equally affects patients with complete and incomplete SCI [79]. A previous EEG study by Vuckovic et al. [86], performed on the same group of volunteers, and the same experimental paradigm, demonstrated a difference in brain response between SCI patients with and without CNP, as well as between both groups of SCI patients and able-bodied volunteers. Those differences were wide spread over the sensory-motor cortex and were not restricted to imagination of paralyzed, painful part of the body. The study was based on ERD/ERS and was primarily interested in a time period after presentation of the directional cue, in a period $t=0.4-2 \mathrm{~s}$.

The MRCP results in the current study are therefore not in accordance with the ERD/ERS analysis on the same patient group [86]. While paralysis resulted in reduced ERD, presence of CNP increased ERD. Therefore that study showed larger difference in cortical response between patients with and with no CNP than between patients with CNP and healthy subjects. The differences were pronounced within the first two seconds after presentation of the directional cue, while in the current study, the difference in MRCP 
morphology was significant in a short interval (0.3 s-0.6 s). This supports the idea of different origin of ERD and MRCP, which has been reported in the literature. The source of MRCP is related to the cerebellar-thalamus-cortical pathway [74][5], while ERD is related to the thalamo-cortical feedback loops [66]. Since CNP is known to be not related to cerebellum activities [86], the difference in the neurophysiological origin of MRCP and ERD supports the observed difference of MRCP and EDR with respect to the presence/absence of CNP.

2) The effect of the Completeness of Injury

Finally patients MRCP were compared on the basis of the completeness of the injury, assuming that presence of CNP does not have a large effect on MRCP. For MRCP measured over $\mathrm{Cz}$ for motor imagery of feet, the largest difference was found in periods of both general preparation and covert movement execution in patients with complete injury.

Castro et al. [16] compared MRCP in chronic paraplegic patients with complete injury and in healthy subjects during covert movement execution of left or right leg. Although in that study larger MRCP could be noticed over electrode locations $\mathrm{C} 3, \mathrm{C} 4$ and $\mathrm{Cz}$, no difference was found when MRCP was averaged over all electrodes. In the current study, we analyzed only electrode location where we expected largest MRCP. We also preprocessed the signal using large Laplacian filter that might have additionally enhances MRCP over these areas.

A general conclusion is that while both CNP and presence/absence of sensa- 
tion affect the morphology of MRCP in paralyzed limb, the factor that most strongly influencs the MRCP is the lack of motor control, resulting in large difference between healthy subjects and general SCI group.

\section{Implications for BCI application}

Results of MRCP classification supported the morphological analysis. In general the highest classification accuracy was found in the time windows which corresponded to the time windows of statistically significant difference between the groups. While classification accuracy between able-bodied group and patients exceeded 90\%, classification between different patients groups was not higher than $65 \%$. This further supports the idea that for MRCPbased BCI systems, paralysis is a factor that needs to be considered as it has a strong influence on the MRCP morphology. Therefore, the following issues should be seriously taken into consideration when developing MRCP-based BCI, especially cue-based BCI, for SCI patients.

Firstly, although the larger magnitude might probably improve the BCI performance in SCI patients, the prolonged rebound should be treated carefully with a long interval between trials. On the other hand, SCI patients with no pain have weaker ERD than the able-bodied volunteers [86], resulting in reduced BCI classification accuracy [65]. This implies that for SCI patients, BCI systems which relay on MRCP might have better classification accuracy, with greater consistency among patients.

Further, the lack of statistical difference of patient sub-groups with the dis- 
tinct peaks in the corresponding p-value curves (lower panels of Figure 5.2) probably resulted from a much larger variability of MRCP in patients (both within and between subjects). This would affect the performance of BCI system for these patients.

Although almost no significant difference was found in the MRCP morphology between patients with pain and with no pain, chronic SCI patients with CNP might experience worsening of pain during prolonged MI practice [29]. Finally, while it was the motor impairment (compared to the remaining sensory function or presence of pain) that had a considerable effect on the MRCP waveforms and can affect BCI performance, the clinical practice and therapy is by no means independent from these factors.

\section{Limitations}

The healthy group, which was comparable to the size of SCI subgroups, was not large. As the magnitude of MRCPs for hand was smaller than that of the foot task (see Figure 5.3), it would be better to have more subjects to increase statistical power of the analysis, so that statistical significance might be revealed in some cases where no significance was detected in the current analysis. Nevertheless, there were 60 trials for each type of task by each subject, so we had hundreds of segments (e.g. 480 for HV and 840 for SCI) for statistical comparison. In fact, we did find significance for HV vs SCI for hand motor imagery (see Figure 5.3), but not for SCI subgroups. Given the very large p-value for PNP vs PWP and CP vs IP (only one peak is close to 
significance level), we believe the likelihood of missing potential significant differences was not large. 


\section{Chapter 6}

\section{Summary and future work}

In this thesis, we presented a series of comprehensive studies on MRCP based BCI applications. We started with two physiological studies in Chapter 2, where we investigated the characters of MRCP morphology under different conditions, i.e. the type of cue and motor task, respectively. Following that we developed algorithms a short-latency brain switch by real-time detection of single-trial MRCP. The results in Chapter 3.1 showed the desired properties of LPP over classic methods, with higher accuracy, lower latency, and subject-independent parameters. In addition, by comparing these key factors that may influence the performance of such a short-latency brain switch, we conclude that ballistic MRCP with time-serial analysis is the optimal solution. Based on this brain switch, in Chapter 4, we proposed two closed-loop

applications. In Chapter 4.1, we implemented a system of brain switch driven mechanical stimulation, aiming at stroke rehabilitation. While in Chapter 
4.2, the brain switch was combined with a electrical stimulation menu, for multi-class BCI applications in communication and motor control. Both systems were intensively tested on healthy subjects, and is ready for clinical investigation. In Chapter 5, a pre-clinical measurement was performed on SCI patients, where we demonstrated the potential application of the proposed closed-loop BCI system on these paralyzed patients.

For the near future, there are mainly two aspects that we need further investigation: 1) the further improvement of the brain switch, and 2) clinical application of the proposed systems.

\subsection{Further improvement of the brain switch}

As demonstrated in Chapter 4, the performance of BCI systems directly relies on the performance of the brain switch. Thus the improvement of the brain switch is a fundamental issue that needs further investigation. Basically the brain switch is a decoder which transfer the input brain signals into commands. Therefore, in order to improve its performance, we could either increase the useful information by adding more features of the input signal, or enhance the decoder's ability in obtaining existing information.

Hybrid brain signal modalities would be the first direction for improving he brain switch's performance. We could make use of the existing brain signals, but utilize more frequency or spatial information. For example, SMRs can be used together with MRCP, which was shown to be able to increase the 
detection accuracy of hand movement [48]. Further, we could increase the number of input channel with other brain signals ,e.g. Electrooculography (EOG). EOG was usually taken as noise in low frequency band of EEG, but it can also be used for hybrid BCI. However, multiple signal modalities would definitely increase the system complexity, as well as computation time, which might be problematic for real-time applications. In addition, the previous studies were performed for hand movement, which was believed to have more spatial information than foot movement, e.g. dorsiflexion in this thesis. Therefore, there issues need to be taken into consideration when we are going to try hybrid signal modalities. On the contrary, we could develop advance machine learning technique for better decoding of the current signal modality. In stead of LPP, there are other manifold learning methods which have been successfully used in complex classification, such as face recognition [34]. One example is Locality Sensitive Discriminant Analysis (LSDA) [47]. Different from LPP, LSDA is a supervised learning method, which constructed two maps for 'signal' and 'noise' respectively. During the projection to lower dimension (as done in LPP), it maximize the margin between the 'signal' and 'noise' samples while at the same time preserve their local information respectively. In this way, the label information in the training data was fully used, which could potentially further increase the TPR and also reduce the FP of the brain switch. 


\subsection{Clinical applications}

The other future work following this thesis is to carry out clinical studies, where we could evaluate the final effect of the proposed systems on target users, e.g. stroke patient.

For a stroke rehabilitation study, a proper experiment proposal would be the first step. There are several issues that need to be carefully treated. The first one is how to design an experiment with proper controls. Apart from the main protocol in Chapter 4.1, we performed two controls in healthy subjects, i.e. BCI alone and MAFO alone. For patient study, a third control group, who only receive classic rehabilitation therapy, would be necessary. This third group could provide the benchmark of spontaneous recover effect. Another issue is the plasticity measurement. MEP measured by TMS, as done for healthy subjects, would also be possible to apply on patients for qualify plastic change. Other technique for plasticity measurement, such as fMRI, would be a supplementary. However, compared with upper limb, the motor context of the foot is relatively small, which might cause difficulty in quantitative measurement. Last but not the least, how to measure the functional change would be a critical issue. As done in [54], the Fugl-Meyer Assessment of the lower limb would be a standard method. Besides, 5 or 10 meters' walking might be an asset for practical measurement, depending on the stage of stroke patients. 


\section{Bibliography}

[1] M. Alegre, A. Labarga, I. G. Gurtubay, J. Iriarte, A. Malanda, and J. Artieda. Movement-related changes in cortical oscillatory activity in ballistic, sustained and negative movements. Experimental Brain Research, 148(1):17-25, Jan. 2003.

[2] K. K. Ang, K. S. G. Chua, K. S. Phua, C. Wang, Z. Y. Chin, C. W. K. Kuah, W. Low, and C. Guan. A randomized controlled trial of EEGbased motor imagery brain-computer interface robotic rehabilitation for stroke. Clinical EEG and Neuroscience, pages 1550059414522229-, Apr. 2014.

[3] K. K. Ang, C. Guan, K. S. G. Chua, B. T. Ang, C. W. K. Kuah, C. Wang, K. S. Phua, Z. Y. Chin, and H. Zhang. A large clinical study on the ability of stroke patients to use an EEG-based motor imagery brain-computer interface. Clinical EEG and Neuroscience, 42(4):253258, Oct. 2011.

[4] K. K. Ang, C. Guan, K. S. Phua, C. Wang, L. Zhou, K. Y. Tang, G. J. Ephraim Joseph, C. W. K. Kuah, and K. S. G. Chua. Braincomputer interface-based robotic end effector system for wrist and hand rehabilitation: results of a three-armed randomized controlled trial for chronic stroke. Frontiers in Neuroengineering, 7:30, Jan. 2014.

[5] C. Babiloni, F. Carducci, F. Cincotti, P. M. Rossini, C. Neuper, G. Pfurtscheller, and F. Babiloni. Human movement-related potentials vs desynchronization of EEG alpha rhythm: a high-resolution EEG study. NeuroImage, 10(6):658-665, 1999.

[6] O. Bai, P. Lin, S. Vorbach, J. Li, S. Furlani, and M. Hallett. Exploration of computational methods for classification of movement inten- 
tion during human voluntary movement from single trial EEG. Clinical Neurophysiology, 118:2637-2655, 2007.

[7] O. Bai, V. Rathi, P. Lin, D. Huang, H. Battapady, D.-Y. Fei, L. Schneider, E. Houdayer, X. Chen, and M. Hallett. Prediction of human voluntary movement before it occurs. Clinical Neurophysiology, 122(2):36472, Feb. 2011.

[8] A. Biasiucci, R. Leeb, A. Al-Khodairy, V. Buhlmann, and J. Millan. Motor recovery after stroke by means of BCI-guided functional electrical stimulation. In Proceedings of the Fifth International BrainComputer Interface Meeting, 2013.

[9] D. C. Boone and S. P. Azen. Normal range of motion of joints in male subjects. The Journal of Bone and Joint Surgery. American Volume, 61(5):756-9, July 1979.

[10] P. Boord, P. J. Siddall, Y. Tran, D. Herbert, J. Middleton, and A. Craig. Electroencephalographic slowing and reduced reactivity in neuropathic pain following spinal cord injury. Spinal Cord, 46(2):118$123,2008$.

[11] A. T. 1. Boye, U. Q. Kristiansen, M. Billinger, O. F. do Nascimento, and D. Farina. Identification of movement-related cortical potentials with optimized spatial filtering and principal component analysis. Biomedical Signal Processing and Control, 3(4):300-304, 2008.

[12] A.-M. Brouwer and J. B. F. van Erp. A tactile P300 brain-computer interface. Frontiers in Neuroscience, 4:19, Jan. 2010.

[13] C. Brunner, M. Naeem, R. Leeb, B. Graimann, and G. Pfurtscheller. Spatial filtering and selection of optimized components in four class motor imagery EEG data using independent components analysis. Pattern Recognition Letters, 28(8):957-964, June 2007.

[14] P. Brunner, S. Joshi, S. Briskin, J. R. Wolpaw, H. Bischof, and G. Schalk. Does the 'P300' speller depend on eye gaze? Journal of Neural Engineering, 7(5):056013, Oct. 2010. 
[15] F. Cassim, W. Szurhaj, H. Sediri, D. Devos, J.-L. Bourriez, I. Poirot, P. Derambure, L. Defebvre, and J.-D. Guieu. Brief and sustained movements: differences in event-related (de)synchronization (ERD/ERS) patterns. Clinical Neurophysiology, 111(11):2032-2039, Nov. 2000.

[16] A. Castro, F. Díaz, and A. Sumich. Long-term neuroplasticity in spinal cord injury patients: a study on movement-related brain potentials. International Journal of Psychophysiology, 87(2):205-14, Feb. 2013.

[17] P. Cisek and J. F. Kalaska. Neural correlates of mental rehearsal in dorsal premotor cortex. Nature, 431(7011):993-6, Oct. 2004.

[18] J. J. Daly and J. R. Wolpaw. Braincomputer interfaces in neurological rehabilitation. The Lancet Neurology, 7:1032-1043, 2008.

[19] O. F. do Nascimento, K. D. Nielsen, and M. Voigt. Relationship between plantar-flexor torque generation and the magnitude of the movement-related potentials. Experimental Brain Research, 160(2):154-65, Jan. 2005.

[20] O. F. do Nascimento, K. D. Nielsen, and M. Voigt. Movementrelated parameters modulate cortical activity during imaginary isometric plantar-flexions. Experimental Brain Research, 171(1):78-90, May 2006.

[21] E. Donchin, K. Spencer, and R. Wijesinghe. The mental prosthesis: assessing the speed of a P300-based brain-computer interface. IEEE Transactions on Rehabilitation Engineering, 8(2):174-179, June 2000.

[22] L. Farwell and E. Donchin. Talking off the top of your head: toward a mental prosthesis utilizing event-related brain potentials. Electroencephalography and Clinical Neurophysiology, 70(6):510-523, Dec. 1988.

[23] R. Fazel-Rezai, B. Z. Allison, C. Guger, E. W. Sellers, S. C. Kleih, and A. Kübler. P300 brain computer interface: current challenges and emerging trends. Frontiers in Neuroengineering, 5:14, Jan. 2012.

[24] S. Filipović, M. Jahanshahi, and J. Rothwell. Uncoupling of contingent negative variation and alpha band event-related desynchronization in a go/no-go task. Clinical Neurophysiology, 112(7):1307-1315, July 2001. 
[25] E. V. C. Friedrich, R. Scherer, and C. Neuper. Long-term evaluation of a 4-class imagery-based brain-computer interface. Clinical Neurophysiology, 124(5):916-27, May 2013.

[26] E. V. C. Friedrich, R. Scherer, and C. Neuper. Stability of event-related (de-) synchronization during brain-computer interface-relevant mental tasks. Clinical Neurophysiology, 124(1):61-9, Jan. 2013.

[27] G. Garipelli, R. Chavarriaga, and J. Millan. Single trial analysis of slow cortical potentials: a study on anticipation related potentials. Journal of Neural Engineering, (10):036014, 2013.

[28] gTec GmbH. IntendiX, 2014.

[29] S. M. Gustin, P. J. Wrigley, L. A. Henderson, and P. J. Siddall. Brain circuitry underlying pain in response to imagined movement in people with spinal cord injury. Pain, 148(3):438-45, Mar. 2010.

[30] M. Haanpää, N. Attal, M. Backonja, R. Baron, M. Bennett, D. Bouhassira, G. Cruccu, P. Hansson, J. A. Haythornthwaite, and G. D. Iannetti. NeuPSIG guidelines on neuropathic pain assessment. Pain, 152(1):1427, 2011.

[31] G. Hagemann, C. Redecker, T. Neumann-Haefelin, H. J. Freund, and O. W. Witte. Increased long-term potentiation in the surround of experimentally induced focal cortical infarction. Annals of Neurology, 44(2):255-8, Aug. 1998.

[32] Y. Hashimoto and J. Ushiba. EEG-based classification of imaginary left and right foot movements using beta rebound. Clinical Neurophysiology, 124(11):2153-60, Nov. 2013.

[33] X. He and P. Niyogi. Locality Preserving Projections. Advances in Neural Information Processing Systems, 16:153-160, 2003.

[34] X. He, S. Yan, and Y. Hu. Face recognition using laplacianfaces. IEEE Transactions on Pattern Analysis and Machine Intelligence, 27:328340, 2005.

[35] D. O. Hebb. The organization of behavior: A neuropsychological theory, volume 44. 1949. 
[36] S. Holm. A simple sequentially rejective multiple test procedure. Scandinavian Journal of Statistics, 6(2):65-70, 1979.

[37] J. Ibáñez, J. I. Serrano, M. D. del Castillo, E. Monge-Pereira, F. Molina-Rueda, I. Alguacil-Diego, and J. L. Pons. Detection of the onset of upper-limb movements based on the combined analysis of changes in the sensorimotor rhythms and slow cortical potentials. Journal of Neural Engineering, 11(5):056009, Oct. 2014.

[38] I. Iturrate, R. Chavarriaga, L. Montesano, J. Minguez, and J. Millán. Latency correction of event-related potentials between different experimental protocols. Journal of Neural Engineering, 11(3):036005, June 2014.

[39] M. Jahanshahi and M. Hallett. The Bereitschaftspotential: movementrelated cortical potentials. Springer, 2003.

[40] N. Jiang, N. Mrachacz-Kersting, R. Xu, K. Dremstrup, and D. Farina. An accurate, versatile, and robust brain switch for neurorehabilitation. In The Annual BCI Research Award, 2013.

[41] M. Jochumsen, I. K. Niazi, N. Mrachacz-Kersting, D. Farina, and K. Dremstrup. Detection and classification of movement-related cortical potentials associated with task force and speed. Journal of Neural Engineering, 10(5):056015, Oct. 2013.

[42] G. Kwakkel, B. J. Kollen, and H. I. Krebs. Effects of robot-assisted therapy on upper limb recovery after stroke: a systematic review. Neurorehabilitation and Neural Repair, 22(2):111-21, Jan. 2007.

[43] M. Lacourse, M. Cohen, K. Lawrence, and D. Romero. Cortical potentials during imagined movements in individuals with chronic spinal cord injuries. Behavioural Brain Research, 104(1-2):73-88, Oct. 1999.

[44] B. Lange, S. Flynn, and A. Rizzo. Initial usability assessment of off-theshelf video game consoles for clinical game-based motor rehabilitation. Physical Therapy Reviews, 14(5):355-363, Oct. 2009.

[45] E. Lew, R. Chavarriaga, S. Silvoni, and J. Millan. Detection of selfpaced reaching movement intention from EEG signals. Frontiers in Neuroengineering, 5:1-17, 2012. 
[46] X. Li, P. Zhou, and A. S. Aruin. Teager-Kaiser energy operation of surface EMG improves muscle activity onset detection. Annals of Biomedical Engineering, 35(9):1532-8, Sept. 2007.

[47] C. Lin, B. Wang, N. Jiang, R. Xu, N. Mrachacz-Kersting, and D. Farina. Discriminative manifold learning based detection of movementrelated cortical potentials for BCI applications. IEEE Transactions on Neural Systems and Rehabilitation Engineering (in press), 2016.

[48] E. López-Larraz, L. Montesano, A. Gil-Agudo, and J. Minguez. Continuous decoding of movement intention of upper limb self-initiated analytic movements from pre-movement EEG correlates. Journal of Neuroengineering and Rehabilitation, 11(1):153, Nov. 2014.

[49] J. N. Mak, Y. Arbel, J. W. Minett, L. M. McCane, B. Yuksel, D. Ryan, D. Thompson, L. Bianchi, and D. Erdogmus. Optimizing the P300based brain-computer interface: current status, limitations and future directions. Journal of Neural Engineering, 8(2):025003, Apr. 2011.

[50] R. J. Marino, T. Barros, F. Biering-Sorensen, S. P. Burns, W. H. Donovan, D. E. Graves, M. Haak, L. M. Hudson, and M. M. Priebe. International standards for neurological classification of spinal cord injury. The Journal of Spinal Cord Medicine, 26:S50, 2003.

[51] D. J. McFarland, L. M. McCane, S. V. David, and J. R. Wolpaw. Spatial filter selection for EEG-based communication. Electroencephalography and Clinical Neurophysiology, 103(3):386-394, Sept. 1997.

[52] N. Mrachacz-Kersting, N. Jiang, K. Dremstrup, and D. Farina. Coupling of motor imagination and nervous system stimulation to induce cortical plasticity. In 5th International Conference on Non-invasive Brain Stimulation, Leipzig,Germany, 2013.

[53] N. Mrachacz-Kersting, S. R. Kristensen, I. K. Niazi, and D. Farina. Precise temporal association between cortical potentials evoked by motor imagination and afference induces cortical plasticity. The Journal of Physiology, 590(Pt 7):1669-82, Apr. 2012.

[54] N. Mrachacz-Kersting, I. K. Niazi, N. Jiang, A. M. Pavlovic, Radovanović, V. S., Kostic, K. Dremstrup, and D. Farina. A novel 
brain-computer interface for chronic stroke patients. In Converging Clinical and Engineering Research on Neurorehabilitation, pages 837841. Springer Berlin Heidelberg, 2013.

[55] G. R. Müller-Putz, V. Kaiser, T. Solis-Escalante, and G. Pfurtscheller. Fast set-up asynchronous brain-switch based on detection of foot motor imagery in 1-channel EEG. Medical \& Biological Engineering \&6 Computing, 48(3):229-233, Mar. 2010.

[56] G. R. Müller-Putz, R. Scherer, C. Neuper, and G. Pfurtscheller. Steady-state somatosensory evoked potentials: suitable brain signals for brain-computer interfaces? IEEE Transactions on Neural Systems and Rehabilitation Engineering, 14(1):30-7, Mar. 2006.

[57] A. R. Murguialday, J. Hill, M. Bensch, S. Martens, S. Halder, F. Nijboer, B. Schoelkopf, N. Birbaumer, and A. Gharabaghi. Transition from the locked in to the completely locked-in state: a physiological analysis. Clinical Neurophysiology, 122(5):925-33, May 2011.

[58] I. K. Niazi, N. Jiang, M. Jochumsen, J. r. F. k. Nielsen, K. Dremstrup, and D. Farina. Detection of movement-related cortical potentials based on subject-independent training. Medical \& Biological Engineering \& Computing, 2013.

[59] I. K. Niazi, N. Jiang, O. Tiberghien, J. r. F. k. Nielsen, K. Dremstrup, and D. Farina. Detection of movement intention from single-trial movement-related cortical potentials. Journal of Neural Engineering, 8:066009, 2011.

[60] I. K. Niazi, N. Mrachacz-Kersting, N. Jiang, K. Dremstrup, and D. Farina. Peripheral electrical stimulation triggered by self-paced detection of motor intention enhances motor evoked potentials. IEEE Transactions on Neural Systems and Rehabilitation Engineering, 20(4):595604, July 2012.

[61] J. B. Nielsen and T. Sinkjaer. Afferent feedback in the control of human gait. Journal of Electromyography and Kinesiology, 12:213-217, 2002.

[62] F. Nijboer, A. Furdea, I. Gunst, J. Mellinger, D. J. McFarland, N. Birbaumer, and A. Kübler. An auditory braincomputer interface (BCI). Journal of Neuroscience Methods, 167(1):43-50, 2008. 
[63] R. Ortner, B. Z. Allison, G. Korisek, H. Gaggl, and G. Pfurtscheller. An SSVEP BCI to control a hand orthosis for persons with tetraplegia. IEEE Transactions on Neural Systems and Rehabilitation Engineering, 19:1-5, 2011.

[64] P. H. Peckham and J. S. Knutson. Functional electrical stimulation for neuromuscular applications. Annual Review of Biomedical Engineering, 7:327-60, Jan. 2005.

[65] G. Pfurtscheller, P. Linortner, R. Winkler, G. Korisek, and G. R. Müller-Putz. Discrimination of motor imagery-induced EEG patterns in patients with complete spinal cord injury. Computational Intelligence and Neuroscience, 2009:104180, 2009.

[66] G. Pfurtscheller and F. H. Lopes Da Silva. Event-related EEG/MEG synchronization and desynchronization: basic principles. Clinical Neurophysiology, 110:1842-1857, 1999.

[67] G. Pfurtscheller and T. Solis-Escalante. Could the beta rebound in the EEG be suitable to realize a "brain switch"? Clinical Neurophysiology, 120(1):24-29, Jan. 2009.

[68] F. Piccione, F. Giorgi, P. Tonin, K. Priftis, S. Giove, S. Silvoni, G. Palmas, and F. Beverina. P300-based brain computer interface: reliability and performance in healthy and paralysed participants. Clinical Neurophysiology, 117(3):531-537, 2006.

[69] F. Pichiorri, F. De Vico Fallani, F. Cincotti, F. Babiloni, M. Molinari, S. C. Kleih, C. Neuper, A. Kübler, and D. Mattia. Sensorimotor rhythm-based brain-computer interface training: the impact on motor cortical responsiveness. Journal of Neural Engineering, 8(2):025020, Apr. 2011.

[70] D. Planelles, E. Hortal, A. Costa, A. Ubeda, E. Iáez, and J. M. Azorín. Evaluating classifiers to detect arm movement intention from EEG signals. Sensors (Basel, Switzerland), 14(10):18172-86, Jan. 2014.

[71] J. Polich. Updating P300: an integrative theory of P3a and P3b. Clinical Neurophysiology, 118(10):2128-2148, 2007. 
[72] J. L. Pons. Rehabilitation exoskeletal robotics. The promise of an emerging field. IEEE Engineering in Medicine and Biology Magazine, 29(3):57-63, Jan. 2010.

[73] A. Ramos-Murguialday, D. Broetz, M. Rea, L. Läer, O. Yilmaz, F. L. Brasil, G. Liberati, M. R. Curado, E. Garcia-Cossio, A. Vyziotis, W. Cho, M. Agostini, E. Soares, S. Soekadar, A. Caria, L. G. Cohen, and N. Birbaumer. Brain-machine interface in chronic stroke rehabilitation: a controlled study. Annals of Neurology, 74(1):100-8, July 2013.

[74] I. Rektor, M. Bareš, and D. Kubová. Movement-related potentials in the basal ganglia: a SEEG readiness potential study. Clinical Neurophysiology, 112(11):2146-2153, 2001.

[75] A. Riccio, D. Mattia, L. Simione, M. Olivetti, and F. Cincotti. Eye-gaze independent EEG-based brain-computer interfaces for communication. Journal of Neural Engineering, 9(4):045001, Aug. 2012.

[76] H. Scheel, R. Xu, N. Jiang, N. Mrachacz-Kersting, K. Dremstrup, and D. Farina. Influence of external cues on synchronized brain-computer interface based on movement related cortical potentials. In 7th IEEE EMBS Neural Engineering Conference, 2015.

[77] E. W. Sellers, D. J. Krusienski, D. J. McFarland, T. M. Vaughan, and J. R. Wolpaw. A P300 event-related potential brain-computer interface $(\mathrm{BCI})$ : the effects of matrix size and inter stimulus interval on performance. Biological Psychology, 73(3):242-52, Oct. 2006.

[78] J. J. Shih, D. J. Krusienski, and J. R. Wolpaw. Brain-computer interfaces in medicine. Mayo Clinic Proceedings, 87:268-279, 2012.

[79] P. J. Siddall, J. M. McClelland, S. B. Rutkowski, and M. J. Cousins. A longitudinal study of the prevalence and characteristics of pain in the first 5 years following spinal cord injury. Pain, 103(3):249-257, 2003.

[80] T. Sinkjaer, J. B. Andersen, M. Ladouceur, L. O. Christensen, and J. B. Nielsen. Major role for sensory feedback in soleus EMG activity in the stance phase of walking in man. The Journal of Physiology, 523:817-827, 2000. 
[81] W.-P. Teo and E. Chew. Is motor-imagery brain-computer interface feasible in stroke rehabilitation? $P M \& R, 6(8): 723-8$, Aug. 2014.

[82] C. Toro, G. Deuschl, R. Thatcher, S. Sato, C. Kufta, and M. Hallett. Event-related desynchronization and movement-related cortical potentials on the ECoG and EEG. Electroencephalography and Clinical Neurophysiology/evoked potentials section, 93(5):380-389, Oct. 1994.

[83] Y. Tran, P. Boord, J. Middleton, and A. Craig. Levels of brain wave activity $(8-13 \mathrm{~Hz})$ in persons with spinal cord injury. Spinal Cord, 42(2):73-9, Feb. 2004.

[84] R. Ulrich, H. Leuthold, and W. Sommer. Motor programming of response force and movement direction. Psychophysiology, 35(6):721-728, Nov. 1998.

[85] A. Venkatakrishnan, G. E. Francisco, and J. L. Contreras-Vidal. Applications of BrainMachine Interface Systems in Stroke Recovery and Rehabilitation. Current Physical Medicine and Rehabilitation Reports, 2(2):93-105, Apr. 2014.

[86] A. Vuckovic, M. A. Hasan, M. Fraser, B. A. Conway, B. Nasseroleslami, and D. B. Allan. Dynamic Oscillatory Signatures of Central Neuropathic Pain in Spinal Cord Injury. The Journal of Pain, 2014.

[87] W. Walter, R. Cooper, V. J. Aldridge, W. C. McCallum, and A. L. Winter. Contingent negative variation: an electric sign of sensori-motor association and expectancy in the human brain. Nature, 203:380-384, 1964 .

[88] P. T. Wang, C. E. King, L. A. Chui, A. H. Do, and Z. Nenadic. Selfpaced brain-computer interface control of ambulation in a virtual reality environment. Journal of Neural Engineering, 9(5):056016, Oct. 2012 .

[89] J. R. Wolpaw, N. Birbaumer, D. J. McFarland, G. Pfurtscheller, and T. M. Vaughan. Braincomputer interfaces for communication and control. Clinical Neurophysiology, 113(6):767-791, June 2002.

[90] World Health Organization. Neurological disorders: public health challenges. Technical report, 2006. 
[91] World Health Organization. The 10 leading causes of death in the world, 2000 and 2011. Technical report, 2013.

[92] R. Xu, N. Jiang, S. Dosen, C. Lin, N. Mrachacz-Kersting, K. Dremstrup, and D. Farina. Endogenous sensory discrimination and selection by a fast brain switch for a high transfer rate brain-computer interface. IEEE Transactions on Neural Systems and Rehabilitation Engineering (in press), 2016.

[93] R. Xu, N. Jiang, C. Lin, N. Mrachacz-Kersting, K. Dremstrup, and D. Farina. Enhanced low-latency detection of motor intention from EEG for closed-loop brain-computer interface applications. IEEE Transactions on Biomedical Engineering, 61(2):288-96, Feb. 2014.

[94] R. Xu, N. Jiang, N. Mrachacz-Kersting, K. Dremstrup, and D. Farina. Comparison of sensory-motor rhythm and movement related cortical potential during ballistic and repetitive motor imagery: an initial report. In 3rd International Conference on Rehabilitation and Medical Engine, 2014.

[95] R. Xu, N. Jiang, N. Mrachacz-Kersting, K. Dremstrup, and D. Farina. Factors of influence on the performance of a short-latency non-invasive brain switch: evidence in healthy individuals and implication for motor function rehabilitation. Frontiers in Neuroscience, 9, 2015.

[96] R. Xu, N. Jiang, N. Mrachacz-Kersting, C. Lin, G. Asín, J. C. Moreno, J. L. Pons, K. Dremstrup, and D. Farina. A closed-loop brain-computer interface triggering an active ankle-foot orthosis for inducing cortical neural plasticity. IEEE Transactions on Biomedical Engineering, 61(7):2092-2101, 2014.

[97] R. Xu, N. Jiang, A. Vuckovic, M. Hasan, N. Mrachacz-Kersting, D. Allan, M. Fraser, B. Nasseroleslami, B. Conway, K. Dremstrup, and D. Farina. Movement-related cortical potentials in paraplegic patients: abnormal patterns and considerations for BCI-rehabilitation. Frontiers in Neuroengineering, 7:35, Jan. 2014.

[98] H. Yang, C. Guan, C. C. Wang, and K. K. Ang. Detection of motor imagery of brisk walking from electroencephalogram. Journal of Neuroscience Methods, June 2014. 
[99] L. Yang, H. Leung, D. A. Peterson, T. J. Sejnowski, and H. Poizner. Toward a semi-self-paced EEG brain computer interface: decoding initiation state from non-initiation state in dedicated time slots. PloS ONE, 9(2):e88915, Jan. 2014.

[100] H. Yuan and B. He. Brain-Computer Interfaces Using Sensorimotor Rhythms: Current State and Future Perspectives. IEEE Transactions on Biomedical Engineering, 61(5):1425-1435, 2014.

[101] P. Yuan, X. Gao, B. Allison, Y. Wang, G. Bin, and S. Gao. A study of the existing problems of estimating the information transfer rate in online brain-computer interfaces. Journal of Neural Engineering, 10(2):026014, Apr. 2013.

[102] X. Zhang and P. Zhou. Sample entropy analysis of surface EMG for improved muscle activity onset detection against spurious background spikes. Journal of Electromyography and Kinesiology, 2012. 


\section{Vita}

\section{Ren $\mathrm{Xu}$}

Add: Von-Siebold-Str. 6, 37075 Göttingen, Germany

Tel: (+49) 1639497821 Email: renxu2012@gmail.com

\section{Education:}

2009 - 2012 M.Sc. in Signal and Information Processing, Chinese Academy of Sciences

2005 - 2009 B.Sc. in Electronics Information Science and Technology, University of Science and Technology of China

\section{Skills:}

Programming: Matlab, C, and assembly language

Statistic software: Minitap, SAS

Documentation: Microsoft Office, Latex

Language: Chinese (Native), English (Fluent), and German (Conversational)

\section{Professional Activities:}

IEEE Student Member

Reviewer for the following journals: IEEE Journal of Biomedical and Health Informatics, PLoS ONE, Frontiers in Neuroscience, Biomedical Signal Processing and Control, Robotics and Autonomous Systems, Mathematical Problems in Engineering Technical Program Committee, 2014 IEEE International Workshop on Pervasive Computing in Biomedical Engineering

\section{Awards:}

The Annual BCI Research Award: Top 10 Nominee in 2013-2015 


\section{Publications:}

\section{Journals:}

R. Xu, N. Jiang, S. Dosen, C. Lin, N. Mrachacz-Kersting, K. Dremstrup, D. Farina. Endogenous sensory discrimination and selection by a fast brain switch for a high transfer rate brain-computer interface IEEE Transactions on Neural System and Rehabilitation Engineering, 2016 (in press).

C. Lin, B. Wang, N. Jiang, R. Xu, N. Mrachacz-Kersting, D. Farina. Discriminative manifold learning based detection of movement-related cortical potentials for BCI applications, IEEE Transactions on Neural System and Rehabilitation Engineering, 2016 (in press).

R. Xu, N. Jiang, N. Mrachacz-Kersting, K. Dremstrup, D. Farina. Factors of influence on the performance of a short-latency non-invasive brain switch: evidence in healthy individuals and implication for motor function rehabilitation, Frontiers in Neuroscience, vol.9(527), 2015.

R. Xu, N. Jiang, N. Mrachacz-Kersting, C. Lin, G. Asin, J. Moreno, J. Pons, K. Dremstrup, D. Farina. A closed-loop brain-computer interface triggering an active ankle-foot orthosis for inducing cortical neural plasticity. IEEE Transactions on Biomedical Engineering, vol.61(7), pp.2092-2101, 2014. (Featured article)

R. Xu, N. Jiang, C. Lin, N. Mrachacz-Kersting, K. Dremstrup, D. Farina. Enhanced low-latency detection of motor intention from EEG for closed-loop brain-computer interface applications. IEEE Transactions on Biomedical Engineering, vol.61(2), pp.288-96, 2014.

R. Xu, N. Jiang, A. Vuckovic, M. Hasan, N. Mrachacz-Kersting, D. Allan, M. Fraser, B. Nasseroleslami, B. Conway, K. Dremstrup, D. Farina. Movement-related cortical potentials in paraplegic patients: abnormal patterns and considerations for BCI-rehabilitation. Frontiers in Neuroengineering, vol.7(35), 2014

R. Xu, N. Jiang, N. Mrachacz-Kersting, K. Dremstrup, D. Farina: Comparison of sensory-motor rhythm and movement related cortical potential during ballistic and repetitive motor imagery. Chinese Journal of Biomedical Engineering, vol. 23(4), 2014.

Book chapters:

N. Mrachacz-Kersting, L. Yao, S. Gervasio, N. Jiang, B.D. Ebbesen, T.S. Palsson, T.G. Nielsen, E. Kamavuako, R. Xu, D. Falla, K. Dremstrup, D. Farina. A Brain-Computer-Interface to combat musculoskeletal pain. Brain-Computer Interface Research: A State-of-the-Art Summary 5, 2016 
(in press).

N. Mrachacz-Kersting, N. Jiang, S. Aliakbaryhosseinabadi, R. Xu, L. Petrini, R. Lontis, K. Dremstrup, and D. Farina. The changing Brain: Bidirectional learning between algorithm and user. C. Guger, G. Müller-Putz and B. Z. Allison Eds. Brain-Computer Interface Research: A State-of-the-Art Summary 4, 2015.

N. Jiang, N. Mrachacz-Kersting, R. Xu, K. Dremstrup and D. Farina. An accurate, versatile, and robust brain switch for neurorehabilitation. C. Guger, T. Vaughan and B. Z. Allison Eds. Brain-Computer Interface Research: A State-of-the-Art Summary 3, 2014.

Conference Proceedings:

H. Scheel, R. Xu*, N. Jiang, N. Mrachacz-Kersting, K. Dremstrup, D. Farina. Influence of external cues on synchronized brain-computer interface based on motor related cortical potentials. 7th IEEE EMBS Neural Engineering Conference, 2015 (*corresponding author).

R. Xu, N. Jiang, N. Mrachacz-Kersting, K. Dremstrup, D. Farina: Detection of motor intention from scalp EEG during ballistic and repetitive motor imagery. BRAIN Grand Challenges Conference, 2014.

R. Xu, N. Jiang, N. Mrachacz-Kersting, K. Dremstrup, D. Farina: Comparison of sensory-motor rhythm and movement related cortical potential during ballistic and repetitive motor imagery: an initial report. 3rd International Conference on Rehabilitation and Medical Engineering, 2014.

N. Mrachacz-Kersting, N. Jiang, R. Xu, K. Dremstrup, D. Farina: Plasticity following skilled learning and the implications for BCI performance. 6th International Brain-Computer Interface Conference, 2014.

C. Lin, B. Wang, N. Jiang, R. Xu, D. Farina. Discriminative manifold learning based detection of movement-related cortical potentials for BCI applications. Bernstein Conference, 2014.

R. Xu, N. Jiang, G. Asn, J. Moreno, J. Pons, N. Mrachacz-Kersting, D. Farina. An ambulatory BCI-driven orthosis for stroke rehabilitation. 5th International Brain-Computer Interface Meeting, 2013. 